\title{
Unicellular Parasite Motility: A Quantitative Perspective
}

\author{
Dissertation \\ for the award of the degree \\ "Doctor rerum naturalium" \\ Division of Mathematics and Natural Sciences \\ of the Georg-August-Universität Göttingen
}

submitted by

Sravanti Uppaluri

from Hyderabad, India

Göttingen 2011 
Prof. Dr. Thomas Pfohl (Reviewer)

Department of Chemistry,

University of Basel

Prof. Dr. Detlev Schild (Reviewer)

Department of Neurophysiology and Cellular Biophysics, Georg-August-Universität Göttingen

Prof. Dr. Marc Timme

Network Dynamics,

Max Planck Institute for Dynamics and Self-Organization

Date of the oral examination: June 27, 2011 
It is declared that the presented thesis has been written independently and with no other sources and aids than quoted.

Göttingen, 23 May 2011

Sravanti Uppaluri 

To my parents, amma and nanna 



\section{Abstract}

The question of how single cells swim is of primary medical importance - especially in the case of pathogenic parasites. Biochemical and cell biological studies have helped elucidate many of the protein building blocks, and chemical interactions involved in motility. However, a complete understanding of microswimmers necessitates a physical and quantitative understanding of swimming mechanisms. In this context, the motility of the parasite Trypanosoma brucei brucei is characterized in biomimetic environments. Trypanosomes, unicellular parasites, cause deadly diseases in humans and cattle in Africa and South America. They are transferred to a mammalian host through an insect vector and thrive within the blood stream and eventually invade the central nervous system. The parasite propels itself through these diverse environments with the aid of a flagellum.

In a minimal homogeneous nutrient rich environment, cells exhibit one of three motility modes distinguishable by their directional persistence. Directional cells take on a straighter shape, while cells that exhibit little net displacement appear more bent. Ascribing the cell body to a worm like chain, we use the cell end to end distance (from base to tip) as a measure of cell stiffness and find that the elongated shape associated with higher directionality is also correlated with higher stiffness. Cell trajectories show a persistence in average swimming direction on the order of $15 \mathrm{~s}$. Further, correlation analysis using high speed microscopy data of $1 \mathrm{kHz}$ uncovered an additional relaxation time arising from strong body distortions in the range of 20 to $100 \mathrm{~ms}$. Random walk models are formulated to describe the motility modes as well as the fast distortions of the cell body. In polymer networks and more viscous environments such as those found in the extracellular matrix, trypanosomes swimming speed is reduced. However, some directionally persistent trypanosomes are found to tunnel their way through networks with mesh sizes smaller than the diameter of the cell. Other cells show little net movement as shown by scaling analysis and appear to probe the elasticity of the network. We show that the movement of these cells can be used to describe the relative differences in elasticity of actin and collagen networks towards a new concept of 'active microrheology'.

Trypanosomes are found to exhibit a propensity to swim close to containing boundary walls and are highly aligned to these boundaries. Using microfluidic channels, trypanosomes suspended in culture medium subjected to flow experience a lift force away from vessel walls and migrate to the center. Purely hydrodynamic effects arising from the trypanosome's shape and density are distinguished from effects of cell motility by comparing with immobilized trypanosome behaviour. We find that the most striking differences in the behaviour between live and immobilized cells arise at flow velocities below $0.1 \mathrm{~mm} / \mathrm{s}$ (more than ten times the self propelling speed of trypanosomes). In this range of resulting shear stresses, trypanosomes exhibit a velocity dependent oscillatory motion 
- swimming upstream from one side of the channel to the other. Immobilized cells tumble in flow, and unlike active cells, do not exhibit an orientational preference. Replacing the suspending medium with whole blood, does not result in significant differences in the center of mass distribution of trypanosomes. However utilizing a constriction-expansion geometry to mimic the cell free layer near the blood vessel walls, we demonstrate that like white blood cells, trypanosomes are expelled by red blood cells toward the boundaries due to differences in cell stiffness. These studies are pertinent to our understanding of how trypanosomes are able to approach vessel walls and invade membrane barriers, including the blood brain barrier for entry into the central nervous system, despite the high shear stresses of blood flow. The present work demonstrates that a quantitative, physical approach uncovers fascinating details of low Reynolds number swimmers. 


\section{Contents}

Abstract i

\begin{tabular}{lll}
\hline & Introduction & 1
\end{tabular}

1.1 Sleeping sickness and trypanosomiasis . . . . . . . . . . . . . 2

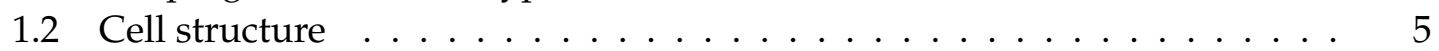

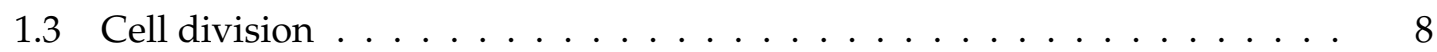

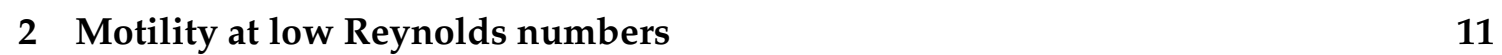

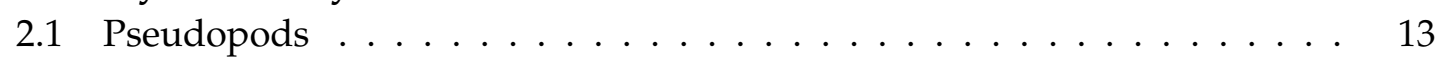

2.2 Ciliary and flagellar movement . . . . . . . . . . . . . . . . . . . 13

2.3 Trypanosome motility $\ldots \ldots \ldots \ldots \ldots$. . . . . . . . . . . . . . 15

\begin{tabular}{lll}
\hline 3 & Random walks & 17
\end{tabular}

3.1 Random walk model . . . . . . . . . . . . . . . . . . . . . . . . . . 17

3.2 Langevin's formulation of Brownian motion . . . . . . . . . . . . . . . 19

3.3 What fits? . . . . . . . . . . . . . . . . . . . . . . . . 19

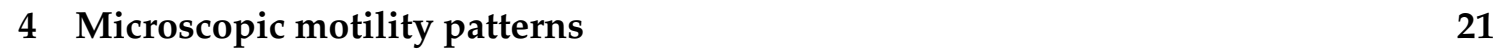

4.1 Experiment . . . . . . . . . . . . . . . . . . . . . 21

4.2 Results . . . . . . . . . . . . . . . . . . . . . . 23

4.3 Modeling trypanosome swimming . . . . . . . . . . . . . 32

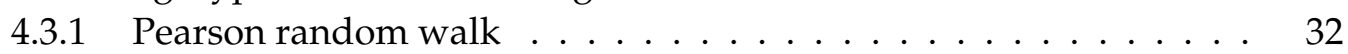

4.3 .2 Langevin equations . . . . . . . . . . . . . . . . . . 34

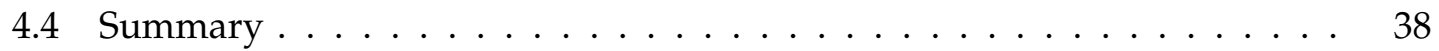

$\begin{array}{|ll|}5 & \text { Motility in tunable environments }\end{array}$

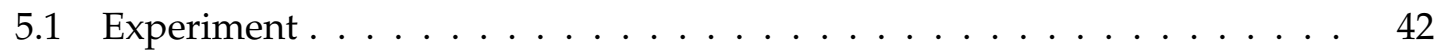

5.1 .1 Dextran preparation $\ldots \ldots \ldots \ldots$. . . . . . . . . . 42

5.1 .2 Actin preparation . . . . . . . . . . . . . . . . . . . 42

5.1.3 Collagen preparation . . . . . . . . . . . . . . . . . 43

5.2 Results . . . . . . . . . . . . . . . . . . . . . 45

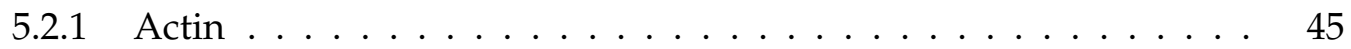

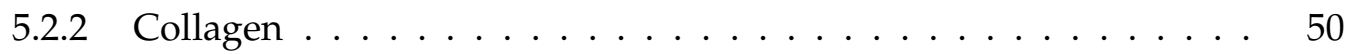

5.2 .3 Dextran . . . . . . . . . . . . . . . . . . . . 53

5.2 .4 Comparison of environments . . . . . . . . . . . . 56

5.2 .5 Trypanosomes as active probes for network properties . . . . . . 58

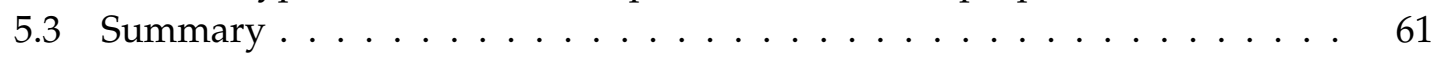


$\begin{array}{lll}6 & \text { Motility in flow } & 63\end{array}$

6.1 Experiment . . . . . . . . . . . . . . . . . . . . . . 64

6.1 .1 Microfluidics . . . . . . . . . . . . . . . . . . 64

6.1 .2 Trypanosome preparation . . . . . . . . . . . . . . . . . 65

6.1 .3 Microscopy and image processing . . . . . . . . . . . . . . 66

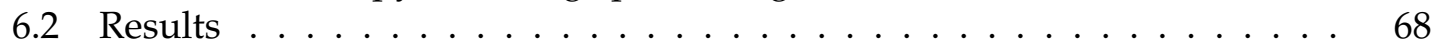

6.2 .1 Sinusoidal trajectories in flow . . . . . . . . . . . . . . . 69

6.2 .2 Center of mass distribution $\ldots \ldots \ldots \ldots$. . . . . . . . . . 71

6.2 .3 Cell elongations . . . . . . . . . . . . . . . . . . 73

6.2 .4 Cell orientation . . . . . . . . . . . . . . . . . . . . 76

6.3 Summary . . . . . . . . . . . . . . . . . . . . 79

\begin{tabular}{llr}
\hline 7 & Motility in biomimetic flow & 81
\end{tabular}

7.1 Blood flow . . . . . . . . . . . . . . . . . . . . 82

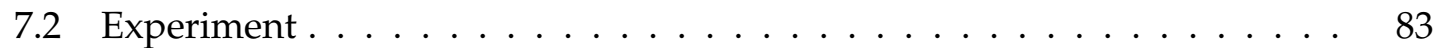

7.2 .1 Preparation of blood cells . . . . . . . . . . . . . . 83

7.2.2 Labeling and detection of trypanosomes . . . . . . . . . . . . 84

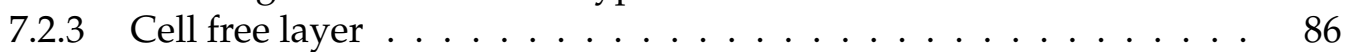

7.3 Results . . . . . . . . . . . . . . . . . . . . . 88

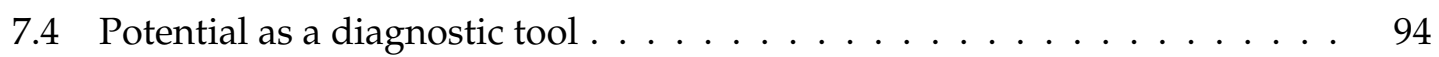

7.5 Summary and outlook $\ldots \ldots \ldots \ldots$. . . . . . . . . . . . . 96

\begin{tabular}{lll}
\hline 8 & Conclusions & 97
\end{tabular}

\begin{tabular}{ll}
\hline List of abbreviations & 100
\end{tabular}

\begin{tabular}{ll}
\hline Bibliography & 101
\end{tabular}

\begin{tabular}{ll}
\hline Publications & 111
\end{tabular}

\begin{tabular}{|l|l}
\hline Acknowledgements & 113
\end{tabular}

\begin{tabular}{ll}
\hline Curriculum Vitae & 115
\end{tabular} 


\section{Introduction}

The cell, often called the fundamental building block of life, is itself comprised of organelles. Organelles, are to the cell what organs are to our body, each serving an essential function. The nucleus is the control center, mitochondria are responsible for energy production, vesicles cargo materials to where they are needed etc. Be it as part of a larger whole in a multicellular organism or as unicellular organism; each cell executes a complex set of actions from maintaining ideal ionic concentrations within the interior of the cell to responding to environmental cues. Likewise, a variety of motility mechanisms and organelles, ranging from tiny surface protrusions, to repeated cycles cell body extrusion and retraction may confer the cell the ability to self propel.

Though cell locomotion has been examined almost since the discovery of the cell

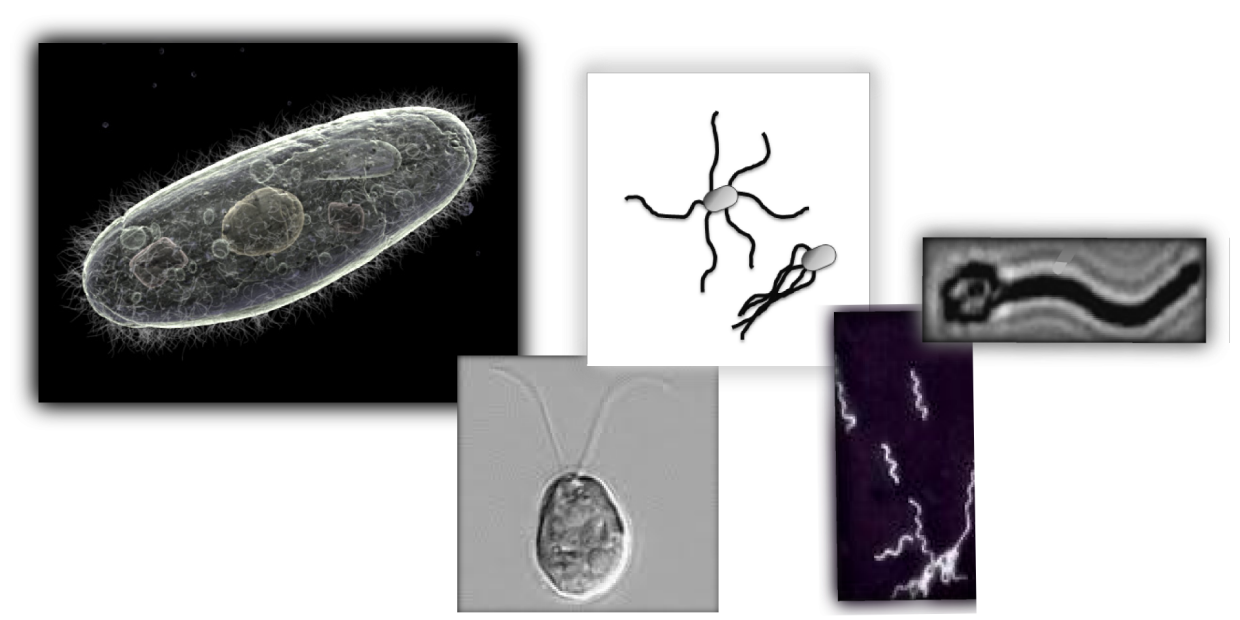

Figure 1.1: Various cell motility mechanisms are known. From left to right: a paramecium swims with surface protrusions called cilia [1], Chlamydomonas - an algal cell swims with two whip-like flagella [2], the E.coli bacterium swims with a bunch of flagella that rotate in the same direction, Spiroplasma, bacterial cells that move by processive changes in body helicity [3], an artificial swimmer whose design is based on sperm cells (comprised of a red blood cell as a head, and a magnetic filament as the tail) is propelled in the presence of a magnetic field [4]. 
itself, advances in microscopy and biochemical studies have paved the way to a more fundamental understanding of cell motility. More recently, a physical, quantitative approach to understanding the world at the microscale has gained momentum. On the whole, these studies provide insight into basic cell biology related to motility, intracellular chemical reactions and external hydrodynamic interactions, allow for modeling of cell movement and may even provide design ideas for artificial microswimmers.

Self-propelled locomotion is essential in the life of many microorganisms ranging from sperm cells, to amoeba, to parasites. Eukaryotic cell motility plays a key role in many physiological functions including, development, survival, as well as disease pathogenesis. Recent work has shown that parasites require cell motility to escape from host immunological attacks and cell division [5]. The ability to swim has been shown to be indispensable for trypanosomes [6], parasitic agents found across the world, having devastating impact on human health and economy [7]. This work is a detailed, quantitative characterization of trypanosome motility.

Here the reader is introduced to the basic biology and epidemiology of trypanosomes. Further details of physical aspects of swimming microorganisms, and the present day knowledge on trypanosome motility is described in Chapter 2. Basic models of diffusion, also applied to cell movement, are introduced in Chapter 3, and then in Chapter 4 , trypanosome swimming in a homogeneous nutrient rich environment and the effect of cell stiffness on the latter is studied. Since trypanosomes are found in diverse environments, we examine trypanosome motility in tunable environments of viscosity and physical polymer networks of actin and collagen in Chapter 5. In Chapter 6, results on the trypanosome behaviour in flow are presented and then in Chapter 7 their behaviour is studied in their native environment - in the blood stream. The thesis ends with a summary of the work.

\subsection{Sleeping sickness and trypanosomiasis}

In Africa two subpsecies, Trypanosoma brucei gambiense and Trypanosoma brucei rhodiesiense, cause human African trypanosomiasis (HAT), commonly known as sleeping sickness in humans. Trypanosoma brucei brucei causes Nagana in cattle. South American trypanosomiasis or Chagas Disease is caused by Trypanosoma cruzi. As shown in Fig. 1.2 the diseases caused by trypanosomes are endemic in low income areas warranting a classification by the World Health Organization as neglected tropical diseases [8].

Like malaria, African trypanosomiasis is transmitted through an insect vector to their hosts. During a blood meal, the Tsetse fly injects the procyclic form (PF) of the parasite into the blood stream. Upon entry into the mammalian host, the parasite morphs to a proliferative blood stream (BSF) form and multiplies spreading throughout the body via the circulatory and lymphatic systems. Some parasites transition to a stumpy, non proliferative form. Stumpy trypanosome can in turn be taken up by the Tsetse fly during 

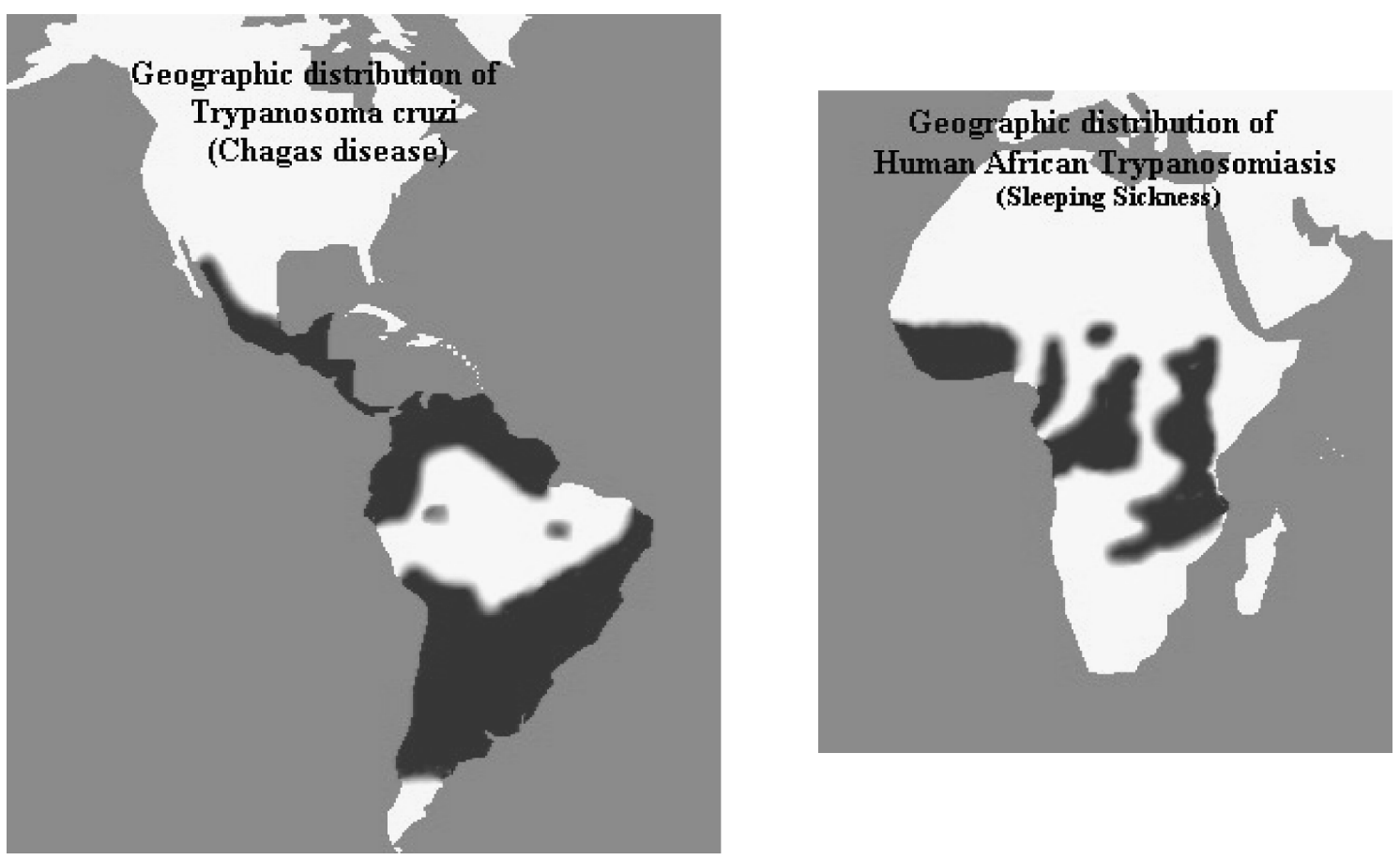

Figure 1.2: Geographical distribution of diseases caused by trypanosomes across the world [9].

a subsequent blood meal. Thus the mammalian host also serves as a reservoir for the parasites. In the Tsetse fly, the trypanosome returns to its PF form and makes an arduous journey toward the salivary glands of its vector host and remains there until the next blood meal. The life cycle of the trypanosome is illustrated in Fig. 1.3 .

Following infection, common flu-like symptoms surface within one to four weeks with subsequent weight loss. Eventually the parasite enters the central nervous system (CNS) causing irritability, personality changes, speech problems, sleep disruption etc, leading to death within weeks to months. Disease prevention programs include both the eradication of the Tsetse fly which may disrupt transmission rates as well as prophylaxis and prompt treatment to reduce the number of people that carry the disease. However, surveillance of the disease is not easy because early symptoms are not evident and testing facilities are not readily available especially in rural settings. Reliable diagnosis can only be made through microscopic examination of chancre fluid, lymph node aspirates, blood, bone marrow, and, during the neurological stage, cerebrospinal fluid. These methods have a high likelihood of false negatives due to low levels of parasitemia and require well trained health professionals.

Currently treatment is given in two stages, during which the patient must be hospitalized. Stage one includes intravenous administration of various combinations of drugs. During the second stage, after parasite entry into the CNS, only melarsoprol and eflornithine (both highly toxic) can pass the blood brain barrier for effective treatment. Often 


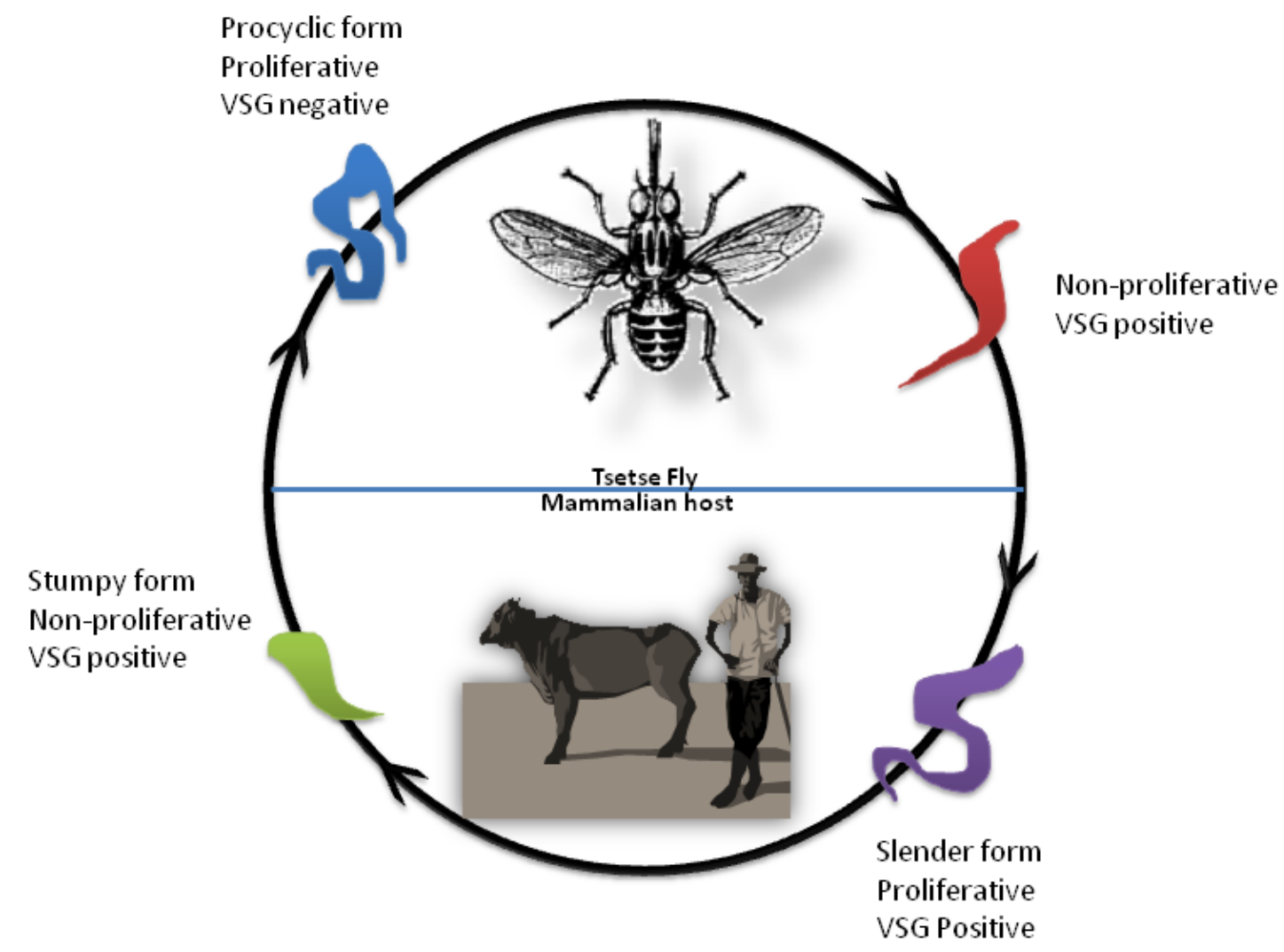

Figure 1.3: Life cycle of the trypanosomes. Illustration of the trypanosomes journey through its vector host and mammalian host. Proliferative status and the presence of variable surface glycoprotein (VSG) is indicated.

treatment can lead to dangerous if not lethal side effects. In all cases the patient has to be monitored for relapse for a minimum of two years. Drug resistant areas do exist presenting an additional challenge to clinicians.

Following a large resurgence in disease occurrence (see Fig. 1.4), in the mid 1990s the World Health Organization, in cooperation with the governments of afflicted countries launched a treatment and prevention campaign resulting in a significant drop in the number of new reported cases of HAT. In 2004, an estimated 66000 new cases were reported across Africa, with 60 million people at risk [10]. Strong research efforts have resulted in improvements in diagnostic tools, as well as the publication of the genome of Trypanosoma brucei. Though these improvements provide tools toward tackling the disease, little progress has been made in disease treatment.

Other major diseases, including Leishmaniasis, caused by similar protozoan parasites are spread throughout the world. The current shortcoming in medical knowledge highlights the need for a better of understanding of these pathogens. Trypanosomes are believed to have been one the earlier eukaryotes to have developed several key features, especially those that involve host-parasite interactions, and RNA editing. RNA 


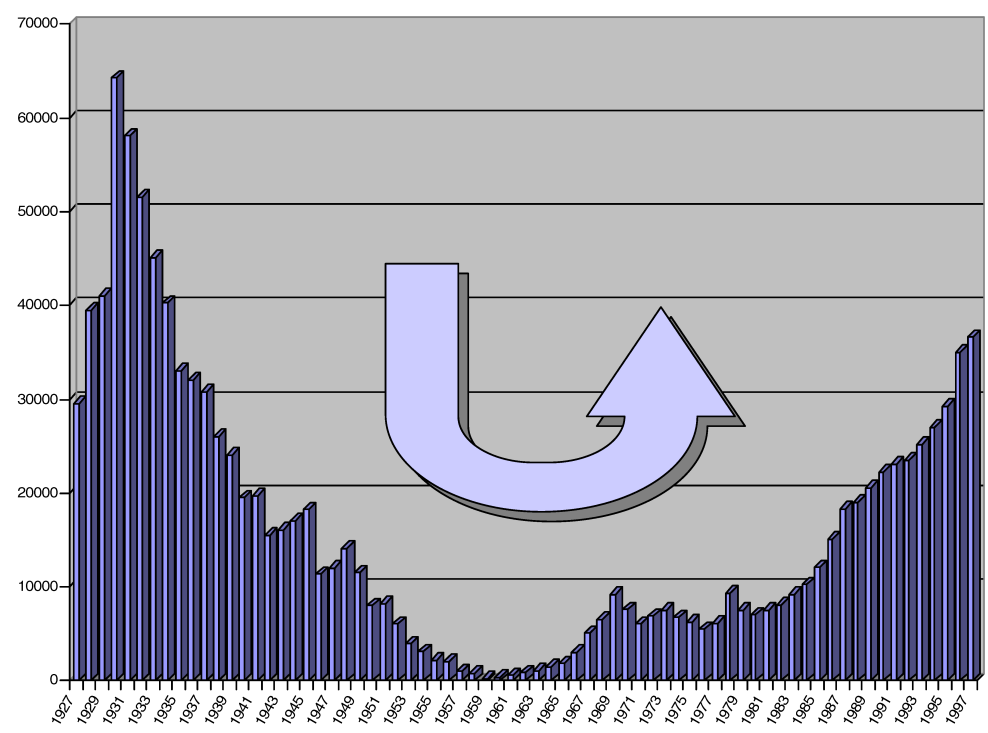

Figure 1.4: Number of new cases of sleeping sickness reported for all Africa between 1927 and 1997, from [7].

(ribonucleic acid) is an intermediate building block from gene to protein. RNA editing is the process by which the base composition can be altered after RNA synthesis. Together, these factors render trypanosomes an interesting and important model for molecular and biophysical studies of unicellular microorganisms.

\subsection{Cell structure}

T.brucei brucei have an elongated cell body 20 to $25 \mu \mathrm{m}$ long with a single flagellum that emerges from the posterior end flagellar pocket and is bound to the entire length of the cell body. As illustrated in Fig. 1.5the cell swims with its anterior end first. Trypanosomes derived their name from the Greek words trypano (borer) and soma (body) because of it was believed that they swim with an auger-like motion. However, a recent study has brought this idea under question; the swimming mechanism of T. brucei brucei is discussed in more detail in later sections of Chapter 2.

The cytoskeleton consists of a corset of over 100 microtubules (MTs) which are linked to each other and to the cell membrane. During cell growth, MTs are generated in between the existing ones thus resulting in semi conservative inheritance of the cytoskeleton [11]. Plus ends of the microtubules are oriented toward the posterior end of the cell conferring an anterior-posterior cell polarity. Other structural elements of the cytoskeleton such as micro and intermediate filaments have not been studied extensively. The flagellum consists of the canonical 9+2 arrangement of MTs and a trypanosome specific feature - the paraflagellar rod (PFR). The PFR is a paracrystalline structure unique to protozoans connecting the flagellar attachment zone (FAZ) to the cell body and pro- 


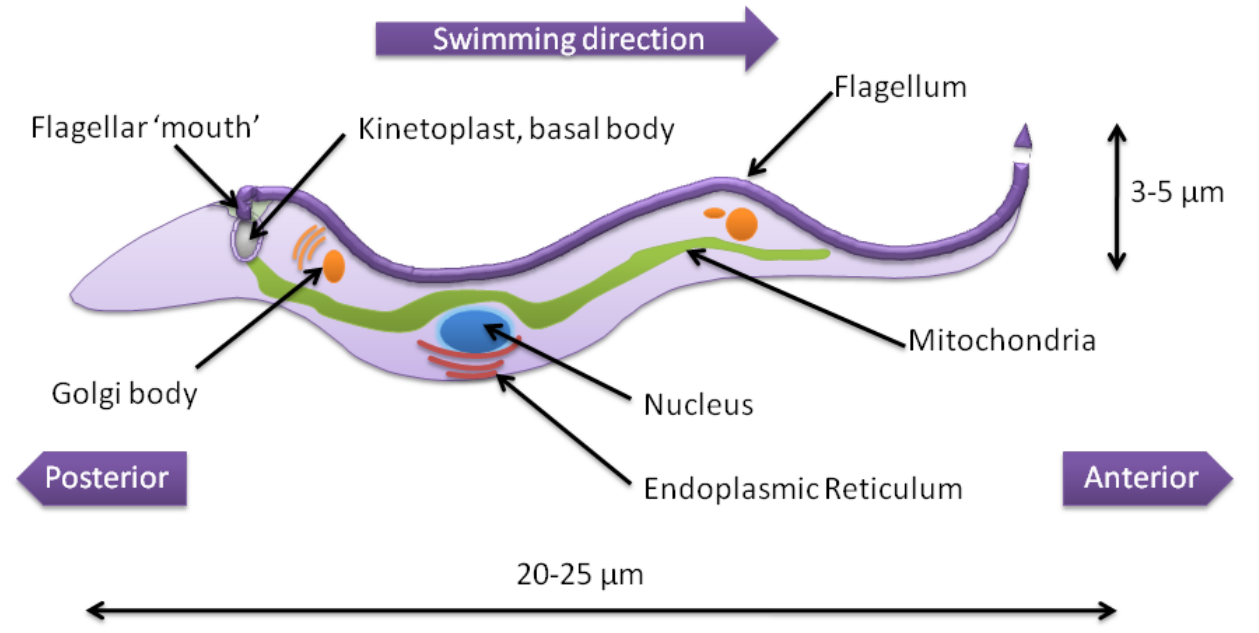

Figure 1.5: Structure and dimensions of T. brucei BSF showing essential organelles.

vides structural support. Attachment of the flagellum is mediated by FAZ composed of a filament complex and MTs shown in Fig. 1.6. Flagellar bending is achieved through adenosinetriphosphate (ATP) mediated sliding of dynein motor proteins between the outer MTs and the inner central MTs. In the absence of ATP the flagellum is relaxed. Other organelles of T. brucei brucei are typical of most eukaryotic cells, including a membrane bound nucleus, mitochondria, endoplasmic reticulum, and golgi apparatus. All these features are illustrated in the figure Fig. 1.5 .

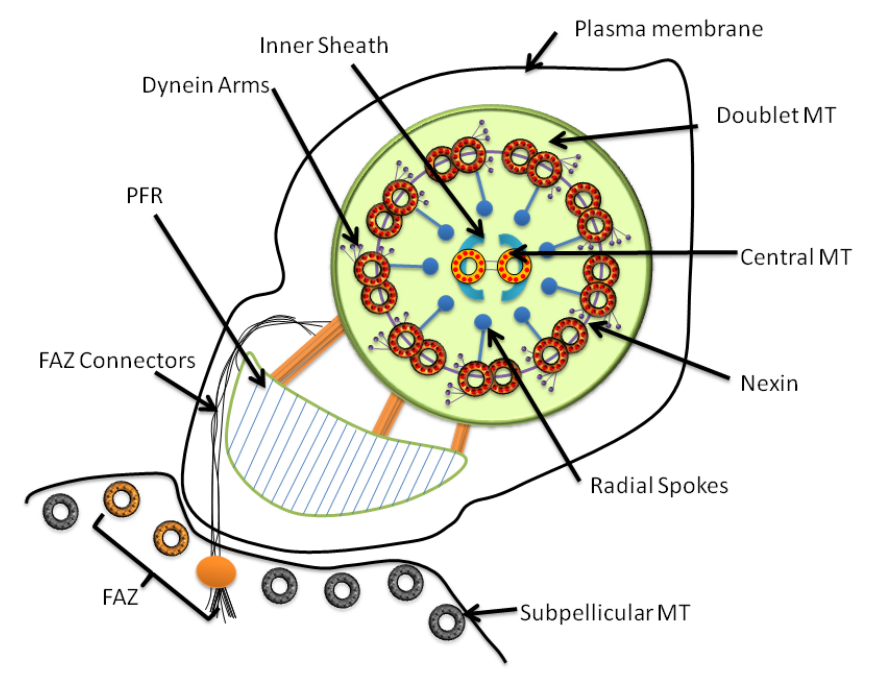

Figure 1.6: Cross section of the trypanosome flagellum and PFR.

A cell surface protein coat plays an essential role in both the bloodstream and pro- 
cyclic form. In the insect stage, the procyclic form of the surface consists of procyclin. In the bloodstream form the cell surface is composed of variable surface glygoprotein (VSG). The thick coat of VSG (VSG positive cell) initiates antibody response from the host but due its density does not allow access to the plasma membrane. Further, the VSG is continually replaced with a modified VSG allowing the 'switchers' to go undetected thereby evading the immune response of the host. Thus once the switchers proliferate, the host is often affected by waves of parasitaemia.

Recent work demonstrated that cell surface hydrodynamic drag is used by trypanosomes to sweep host antibodies to the flagellar pocket, the 'cell mouth', for ingestion [5]. Thus trypanosomes are able to evade the host's immunological response. A genetically modified reverse swimmer was used to show that the hydrodynamic drag effectively sweeps antibodies away from the leading end of the cell. A time series is shown in Fig. 1.7 demonstrating the flow mediated cell surface protein sorting.

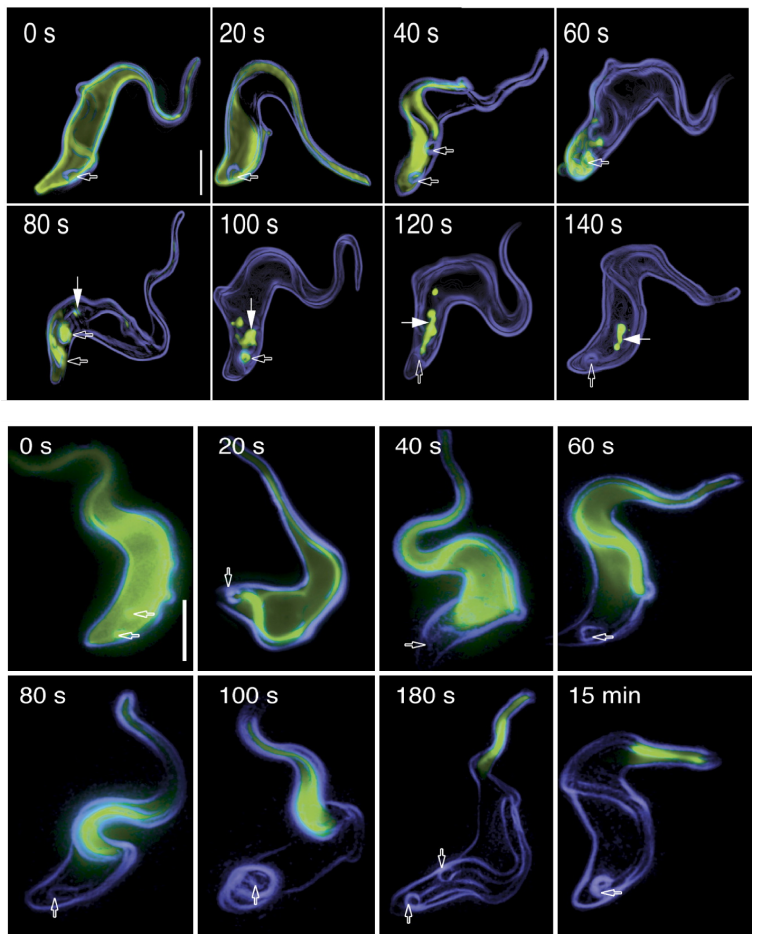

Figure 1.7: Time series showing forward swimmer (top) and reverse swimmers (bottom) using hydrodynamic cell surface drag to sweep antibody proteins (green) away from the leading end of the cell. The forward swimmer ingests the antibodies allowing it to remain undetected by the host's immune system (from [5]).

Two pockets of DNA are found in the cell (Fig. 1.5). The cell nucleus is centrally located and contains 11 pairs of chromosomes, intermediate chromosomes, and $\sim 100$ mini-chromosomes. The mitchondrial DNA, called the kinetoplast, consists of mini and maxi circles of DNA and is found at the base of the flagellar pocket. The kinetoplast also plays a key role in cytoskeletal structure. The number and location of these two DNA 
pockets are characteristic of particular cell division stages, described below.

\subsection{Cell division}

Cell division of blood stream trypanosomes is not as well understood as the procyclic form, thus stage specific differences in cell replication have not been well studied. Nevertheless, as illustrated in Fig. 1.8, a stepwise progression through cell division can be classified by the number of nuclei and kinetoplasts in the cell. Note that bloodstream trypanosomes can divide to the non-proliferating 'stumpy' form which can in turn be re-introduced to the insect vector, the Tsetse Fly. However, most laboratory models differentiation to the stumpy form is negligible [11].

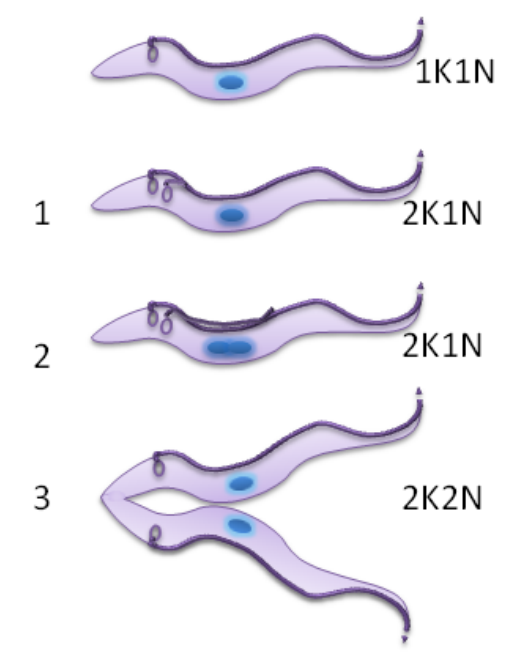

Figure 1.8: Stepwise cell division cycle of T. brucei brucei (described further in the text). The number of kinetoplasts $(\mathrm{K})$ and nuclei $(\mathrm{N})$ are markers of the cell division cycle.

Cell division proceeds as follows:

1. The cell body begins to expand, while the kinetoplast, attached to the basal body is replicated. Division begins with the growth of a new flagellum along the older one.

2. Mitosis of the nucleus begins while the second flagellum continues to grow.

3. Cytokinesis proceeds and the two cells are resolved though they may remain connected at the posterior end for a significant amount of time before a final separation. Following cytokinesis, the two cells may remain attached for a long period of time. It has been suggested that cell motility may play an essential role in 'tearing' the two cells apart.

Thus, inheritance of microtubules and basal body are semi-conservative: each daughter cell inherits newly formed microtubules as well as microtubules formed in the previous cycle. On the other hand, the flagellum is inherited in a semi conservative way: one 
cell receives the old flagellum while the other receives the flagellum formed during the latest cycle. Additionally the flagellum has been postulated to be a sensory tool to detect changes and obstacles in the environment [12; 13].

RNA interference-based ablation of flagellar proteins in bloodstream-form parasites have resulted in loss of cell viability demonstrating the importance of cell motility and in particular the flagellum in cell survival [6]. The discovery of social motility in trypanosomes [14] further motivates a deeper look into their motility. 



\section{Motility at low Reynolds numbers}

Most of the mechanisms familiar to us used for motion in our everyday life, including those of fish, insects and birds would not allow for any net motion for organisms operating at the micrometer scale. Microorganisms inhabit a world that is very different from ours. It is the world of low Reynolds numbers - a world where viscous forces dominate over inertial forces. The Reynolds number, given by

$$
R e=\rho U L / \eta
$$

(where $\rho$ is the fluid density, $U$ is the velocity of the flow, $L$ is the characteristic length scale and $\eta$ is the fluid viscosity), defines the ratio of inertial forces to viscous forces.
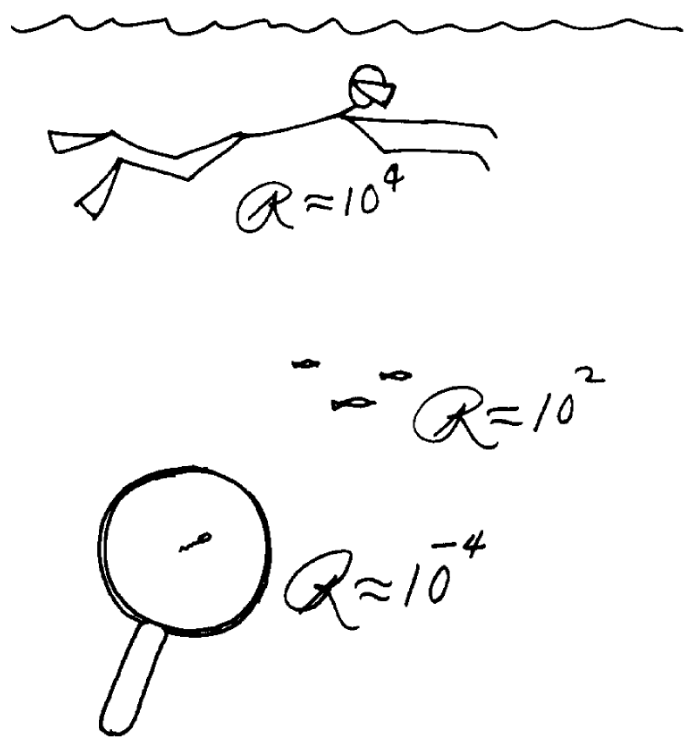

Figure 2.1: Reynolds number for various swimmers, from [15].

Swimmers with $R e<1$ are generally considered to be low Reynolds number swimmers. The physics governing swimming at low Reynolds numbers (micrometer length scales) is significantly different from that of swimming at the macroscopic length scale. While a fish propels itself by accelerating water, the bacterium does so using viscous 
shear.

Swimming is a result of periodic motion. However, an interesting fact about low Reynolds number swimming is that reciprocal motion, in which the motion is reversed through the same reverse sequence, does not result in any net displacement.

For an incompressible Newtonian fluid with density $\rho$ and viscosity $\eta$, the flow satisfies the Navier-Stokes equations,

$$
\rho\left(\frac{\partial u}{\partial t}+(u \nabla) u\right)=-\nabla p+\eta \nabla^{2} u
$$

with flow field $u$ and pressure $p$ in the surrounding. As discussed above, the inertial terms are negligible at low Reynolds number, and we are left with the linear Stokes relation:

$$
-\nabla p+\eta \nabla^{2} u=0
$$

As can be seen from Eq. 2.3. time dependence is lost; only geometry or the sequence of body deformations is relevant in describing the displacement of the body. The so called Scallop theorem arises from just this fact. A scallop moves by opening slowly and closing much faster, squirting out water and resulting in displacement. Due to the absence of time dependence, the reciprocal motion of the Scallop at low Reynolds number would not result in any 'swimming'. A direct consequence of the Scallop theorem is that at low Reynolds numbers movement with at least two degrees of freedom is required to avoid reciprocal motion. The commonly found biological form of non-reciprocal motion is therefore a wave [16], such as the wave produced by most sperm cells.

The interior of any cell is composed of a cytoskeletal fibrous network of three primary components: microtubules, actin microfilaments, and intermediate filaments such as keratin and vimentin. These fibers have diameters of $24 \mathrm{~nm}, \sim 8 \mathrm{~nm}$ and intermediate diameters respectively. These components provide structural support, integrity, and allow for force generation. Both force generation and polymerization of filaments are fueled by ATP or GTP. Motor proteins interact specifically with filaments (as shown in Fig. (1.6); in trypanosomes dynein proteins tread along the microtubules in the flagellum to produce bending and ultimately cell motion.

Besides the obvious biological interest in understanding cell motility, gaining a fundamental understanding of microorganisms may aid in the design and implementation of efficient artificial micromachines [4; 17; 18] for varied applications. Most microorganism swimming is mediated by one or more filaments such as cilia or flagella. In other cases, motility is mediated by deformations of the body itself resulting from changes in the cytoskeleton. In general eurkaryotic appendages are much larger than prokaryotic appendages. The former produces active changes in flagellum shape while the latter rotate their flagella with the use of an active motor. Nevertheless, each species has adapted a different set of 'motility rules' in terms of beat pattern, wavelength, wave frequency 
and flagellar length.

Here we outline well known examples of microorganism mechanisms and finally end with a review of what is known about the motility mechanism of trypanosomes.

\subsection{Pseudopods}

Some types of eukaryotic cells, known as amoeboids, use pseudopods for motility. Cells extend and contract by the assembly and disassembly of actin microfilaments. The cell projects itself forward using cytoplasmic projections which can be used both for cell movement but also to engulf nutrients or prey as illustrated in Fig. 2.2. Other cells such as white blood cells also use the same mechanism for movement and digestion of waste and foreign bodies.

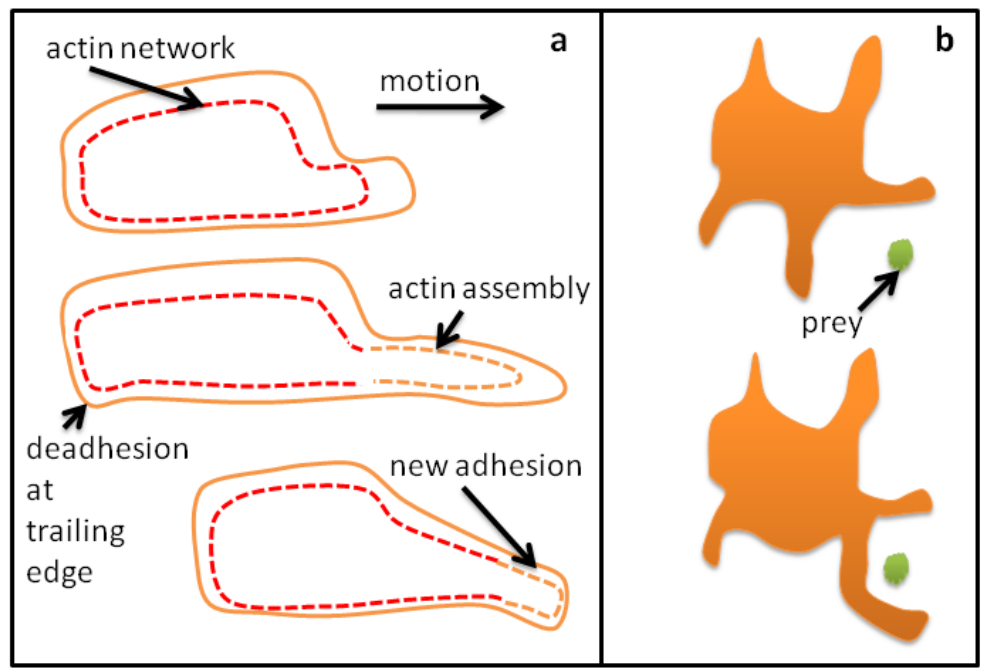

Figure 2.2: a. Schematic of motion mediated by extensions of the cell body. b. Engulfing prey

The forces generated by such cells have been discussed elsewhere [19].

\subsection{Ciliary and flagellar movement}

Both cilia and flagella are composed of the iconic 9+2 microtubule structure [20] shown in Fig. 1.6 and are attached to the cell body via a basal body. The distinguishing feature is that cilia are shorter than flagella and they are generally many cilia per single cell. The motion of the cilia are synchronized on the cell surface to produce a metachronal wave [21]. Ciliary propulsion is used by organisms of different sizes, including: the algae Chlamydomonas reinhardtii, and paramecium (with thousands of cilia). An asymmetric pattern in which the cilium extends and bends at the base during the forward stroke 
and then folds on itself during the recovery stroke results in ciliary propulsion as seen in Fig. 2.3 .

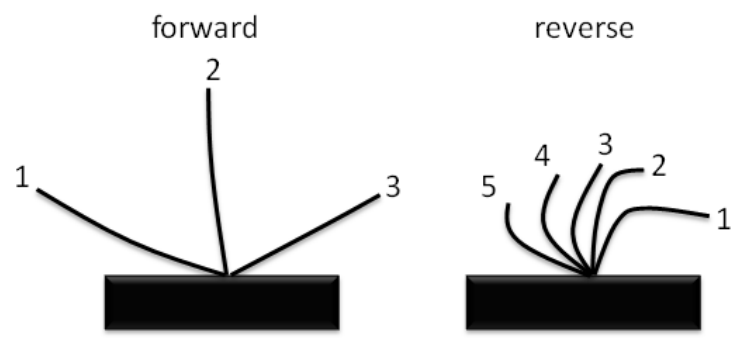

Figure 2.3: Effective stroke (left), and reverse (right) - numbering indicates the order of motion. Note the asymmetric motion.

A eukaryotic flagellate swims by generating either a planar or helical wave by the flagellum. The classical example is that of a sperm cell. A sperm cell, illustrated in Fig. 2.4 is typically composed of a dense 'head' containing genetic material and a flagellar tail that is up to 10-15 times longer. The flagellum, on the order of microns to millimeters in length, may beat in a variety of patterns depending on the species. Both chemotaxis (motion toward or away from a chemical signal) and rheotaxis (motion toward or away from a flow field) have been demonstrated in mammalian sperm cells [22; 23].

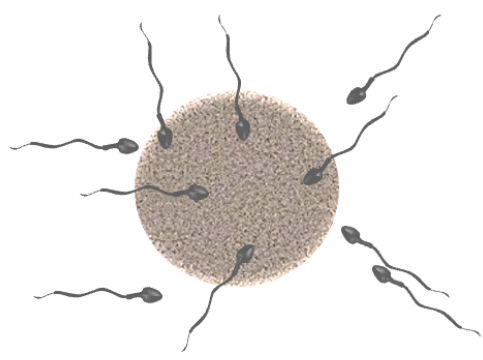

Figure 2.4: Motility is essential for typical sperm cells that must compete to fertilize the egg.

Prokaryotic flagella are attached to the cell body via a basal motor allowing the flagellum to be rotated producing a helical wave. The motility of of E.coli, rod like bacteria measuring 2-3 $\mu \mathrm{m}$ in length and 1-2 $\mu \mathrm{m}$ in width has been extensively studied. They propel themselves forward with several flagella which rotate counterclockwise. However, the flagella can abruptly begin to rotate clockwise resulting a 'tumble' motion with little displacement [24; 25] (see Fig. 2.5). It is believed that E.coli motility has been optimised for chemotaxis [25].

In addition to swimming, both flagella and cilia can act as sensory organs [26]. 


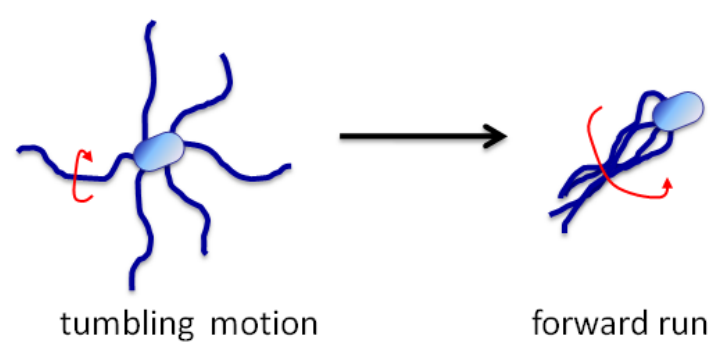

Figure 2.5: E. coli run and tumble motion is determined by the direction of flagellar rotation.

\subsection{Trypanosome motility}

As in other motile cells such as E.coli and spermatozoa, T. brucei brucei motility is mediated by a flagellum. However, unlike sperm and E.coli, the flagellum of the trypanosome emerges from the posterior end flagellar pocket and runs along the length of the entire body as illustrated in Fig. 1.5. Until very recently it was believed that the flagellar beat originates at the tip and is carried to the base of the cell resulting in a corkscrew like forward movement [27; 28; 29], see Fig. 2.6. Branche et al. showed additionally that base to tip wave propagation resulted in reorientation of the whole cell without any significant backward movement [29]. This corkscrew mechanism was also observed in high speed microscopy showing a preferred chirality in cell rotation [30]. However, work by Rodriguez et al. suggests that both blood stream form and procyclic form cells move by two anti-chiral helices separated by a kink that travels along the length of the body during wave propagation [31] as illustrated in Fig. 2.6p.

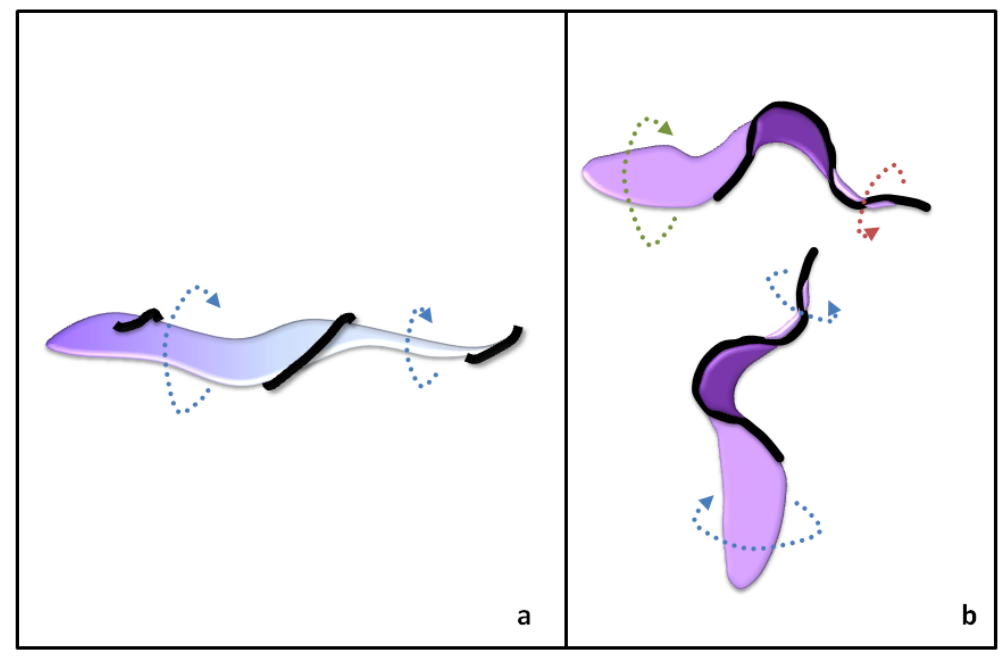

Figure 2.6: a. Previous perception of T. brucei brucei swimming mechanism - corkscrew like behaviour. b. Propogation of antichiral bihelical waves results in cell propulsion. 
By analyzing images from millisecond microscopy, Rodriguez et al. found that the flagellum tip changes its direction of rotation $19 \pm 3$ times per second, producing helical waves of alternating chirality. The broad end of the cell rotates in the opposing direction of the flagellar tip, but this rocking motion appeared to change direction at the rate of $5 \pm 3$ flips per second. As discussed above, a reciprocal motion is not sufficient for swimming at low Reynolds numbers. Therefore, Rodriguez et al. suggested that the difference in rotation frequency at opposing ends of the cell allows for a kink, separating the helical waves with opposing chirality, to travel from the anterior end to the posterior end. Such a mechanism is non-reciprocal and explains the propulsion of the trypanosome cell. Thus, the high rotation frequency would provide the traction force to pull the cell body along, and the reduced rotation rates toward the larger posterior end reduce viscous drag and optimize ATP utilization [31].

A similar mechanism has previously been established for Spiroplasma, prokaryotic organisms without a cell wall [32]. Pairs of kinks travel down the body axis of Spiroplasma for forward propulsion. While Spiroplasma do not possess flagella, they have protofilament ribbons that span the length of the cell to maintain a helical form. In Spiroplasma, the kinking mechanism consists of two temporally distinct types of cell deformations. The first deformation changes the handedness of the cell helix (a right-handed helix becomes left-handed and vice versa) at one end and continues to grow toward the the opposite end of the cell. The second deformation switches the cell back to its initial chirality, after an average of $0.26 \mathrm{~s}$, resulting in a kink that travels the length of the cell to the distal end, thereby resulting in cell propulsion. A model by Wolgemuth et al. showed that such behaviour could in fact result in propulsive force and that increasing fluid viscosity would result in a corresponding increase in swimming velocities [33]. The latter observation was experimentally verified [34].

These contradicting reports show that the trypanosome motility mechanism remains elusive. 


\section{Random walks}

In 1827 Scottish botanist Robert Brown found that pollen particles exhibit a jittery motion and demonstrated that this motion persisted despite treatment with several killing processes. He could not however explain the origin of this temperature dependent motion. In 1905, Albert Einstein published a model for Brownian motion. He obtained a relation between the macroscopic diffusion constant $D$ and the molecular components of matter:

$$
D=\frac{R T}{N_{A} 6 \pi \eta a}=\frac{k_{b} T}{6 \pi \eta a}
$$

where $R$ is the gas constant, $N_{A}=6.06 \times 10^{23} \mathrm{~mol}^{-1}$ is Avogadros number, $T$ is the temperature, $a$ is the radius of the Brownan particle and $k_{b}=R / N_{A}$ is Boltzmann's constant [35].

Simultaneously, Smoluchowski described the time dependence of a Brownian particle's displacement using a probability function. In 1908, Langevin described Brownian motion as a result of a stochastic force pushing a particle in velocity space.

Przibram showed in 1913 that trajectories of individual protozoa also satisfy the relationship that had been established for Brownian motion just a few years before [36].

As such, the theory of continuous random processes in Brownian motion has coevolved with biological motility models and has also made an impact on a diverse range of applications from economics to polymer physics. One such application is in the study of efficient search strategies of animals and microorganisms for food sources. The multitude of target site locations, and the most efficient order in which to visit the target sites presents a rich problem requiring a statistical approach. In this chapter, we briefly review the simplest model of an unbiased uncorrelated random walk - initially utilized to describe Brownian motion and later extended to other applications.

\subsection{Random walk model}

Consider a one-dimensional drunken sailor starting at the origin $(x=0)$ - he is unpredictable with an equal probability of taking a step forward and backward. This is a process Markovian process such that each step is independent of the past. After the first step, the sailor is at a distance $\delta$ to the left or right of the origin. Following the subsequent step, 
the sailor may be either at $2 \delta$ to the left or right of the origin (with probability $1 / 4$ each) or at the origin (with probability $1 / 2$ ). The probability that after $n$ independent steps, a walker will be at a distance $m \delta$ to the right of the origin after $n$ steps is:

$$
\begin{aligned}
p(m, n) & =(1 / 2)^{n}\left(\begin{array}{c}
n \\
\frac{(n-m)}{2}
\end{array}\right) \\
& =\frac{n !}{2^{n}((n+m) / 2) !((n-m) / 2) !}
\end{aligned}
$$

Given, $\tau_{0}$ the time interval after each step takes place (on the order of $10^{-8} \mathrm{~s}$ ), $m=x / \delta$ and $n=t / \tau_{0}$, for large $\mathrm{n}$ ( $\delta$ is infinitesimally small for a finite value of $\mathrm{x}$ ), this equation converges to a Gaussian distribution with mean 0 and variance $\delta^{2} t / \tau$.

$$
P(x) d x=\frac{1}{\sqrt{4 \pi D t}} e^{\left(\frac{-x^{2}}{4 D t}\right)} d x
$$

with $D=\delta^{2} / 2 \tau$. Eq. 3.3 is also a solution to the diffusion equation with $\mathrm{D}$ as a constant known as the diffusion coefficient. Thus, the mean values of $\langle x(t)\rangle=0$ and $\left\langle x^{2}(t)\right\rangle=2 D t$. From the latter, the root mean squared displacement, is experimentally measurable:

$$
x_{\text {rms }}=<x^{2}(t)>^{1 / 2} .
$$

For a 3-dimensional system the above equation becomes

$$
\left\langle r^{2}(t)\right\rangle=6 D t
$$

thus connecting the diffusion constant with the microscopic displacement process.
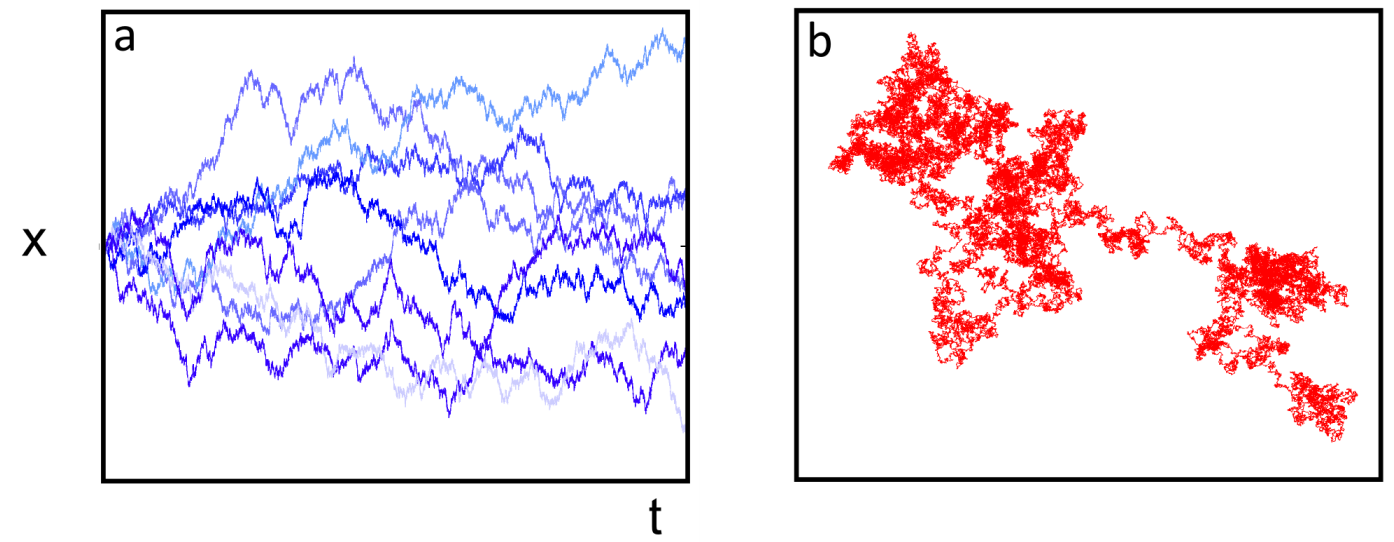

Figure 3.1: a. Eight realizations of a 1-D random walk starting at $\mathrm{x}=0$ plotted as a function of time. b. 2-D random walk (step sizes are drawn from a Gaussian distribution, Matlab).

A correlation between successive steps, termed persistence, has frequently been in- 
troduced to model animal motility using continuous random walks (CRW). Kareiva and Shigesada derived an equation for the mean squared displacement for a two dimensional random walk that includes a variable step length and a specific turn angle distribution [37]. This model is further discussed in the next chapter. Directional bias can also been introduced, in the case of environmental cues or chemical gradients in biased random walks (BRWs) [38: 39].

\subsection{Langevin's formulation of Brownian motion}

Langevin put forth an equivalent theory of Brownian motion using a different approach by applying Newton's second law. He formulated his theory based on the assumption that the particle experiences a time dependent force due to the impact of molecules from the surroundings, without any other external forces. This force arises from a steady force due to frictional drag of the fluid, $\mathbf{f}_{d}$ and the random force $\mathbf{f}_{r}$ due to the collisions with the surrounding fluid molecules. Thus the equation of motion is given by:

$$
M \frac{d \mathbf{v}}{d t}=\mathbf{f}_{d}+\mathbf{f}_{r}
$$

The drag force is given by the Stokes formula such that $\mathbf{f}_{d}=\gamma \mathbf{v}$ where $\gamma$ is the viscous drag coefficient. For a spherical particle of radius $a$ in a fluid of viscosity $\eta$,

$$
\gamma=6 \pi \eta a .
$$

The random force $\mathbf{f}_{r}$ is independent of $\mathbf{v}(t)$ and varies extremely fast compared to it so that on average $\left\langle\mathbf{f}_{r}\right\rangle=0$ and is referred to as a stochastic force.

Eq. 3.6 returns the Einstein relation given in Eq. 3.1 describing the diffusion of a particle in a fluid of given viscosity [40; 38]. Eq. 3.6 may be further modified to explicitly account for external forces, nonlinearities in the viscous drag, higher powers of velocity or some other function of $\mathrm{v}(\mathrm{t})$.

\subsection{What fits?}

In cell motility studies, the advent of computer tracking algorithms, and higher resolution recordings led the way for much more extensive data collection. Agreement between data, and mathematical models could be confirmed, and fundamental assumptions regarding bias and correlation could be verified.

In general it was found, and often assumed that an analogy could be drawn from normal diffusion to animal movement. Random walks, can however be Brownian (such that as $t \rightarrow \infty$, the mean square displacement increases linearly with time $\left.-\left\langle r^{2}(t)\right\rangle \sim t\right)$ or Lévy (such that $\left\langle r^{2}(t)\right\rangle \sim t^{\alpha}$, with $1<\alpha<3$ ). The latter is true when movement is dominated by much longer, but rarer step lengths - without finite variance. While it is 
possible to derive a step length distribution experimentally, a lively debate is ongoing on how to definitely characterize movement as 'Brownian' or 'Lévy' [41; 42: 43]. The debate arises essentially from the significance of the collected statistics, as well as the method of data collection [44]. Nevertheless, a wealth of new studies dealing with topics from human mobility [41; 42] to honeybee search strategies [45] are surfacing and may aid in understanding the diverse behaviours found in nature.

In the next chapter, trypanosome swimming is modeled using two different approaches and characterized in terms of cell stiffness. 


\section{Microscopic motility patterns}

In this chapter we show that differences in cell body stiffness result in a diverse range of cell behaviours contributing to the directional motion of the cell. Stiffer cells swim more directionally while cells that exhibit little net displacement appear to be more flexible. The results point to significant flexural rigidity differences within individuals of the same population. Cell trajectory and rapid body fluctuation correlation analysis uncovers two characteristic relaxation times: a short relaxation time due to strong body distortions in the range of 20 to $100 \mathrm{~ms}$ and a longer time associated with the persistence in average swimming direction on the order of $15 \mathrm{~s}$. Different motility modes resulting from varying body stiffness may be of consequence on host invasion during different infective stages. We present a Pearson random walk model which fully describes the observed motility modes. Finally, a Langevin model is also used to account for the fast body undulations of the cell body.

While there have been many efforts to uncover the molecular biology of motility in trypanosomes and other microorganisms, a quantitative understanding of parasite motility is still lacking. All studies of trypanosome motility have focused exclusively on cells swimming directionally [27; 31]. However, it is known that more than half of a trypanosome population is dividing under normal culture conditions underscoring the importance of carrying out population-wide analysis for quantitative characterization. We aim not only to characterize differences in swimming behaviour within a single population, but also to link these behaviours to the microscopic physical processes that may explain these observations. Studies of single cell trajectories have revealed many details of cell motility of other organisms such as spermatozoa, E.coli, and Dictyostelium discoideum. To this end, we examined motility of single trypanosomes in a two-dimensional environment.

\subsection{Experiment}

Trypanosoma brucei brucei grown in HMI9 complete culture medium [46:47] supplemented with $10 \%$ fetal calf serum (FCS) were cultured and harvested at a cell density of $2 \times 10^{5}$ cells $/ \mathrm{mL}$. Cells were resuspended in fresh culture medium before every tracking experi- 
ment. Cell swimming was observed between two microscope slides in HMI-9 complete medium in a 2-dimensional setting between a microscope slide and a coverslip (Fig.4.1), precleaned by sonication in isopropanol and dried with high pressure.

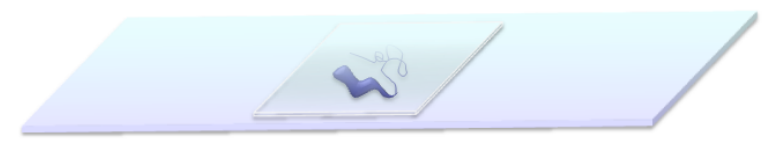

Figure 4.1: Isolated single cells were observed between two glass slides with a 10 $\mu \mathrm{m}$ gap.

An Olympus BX61 upright microscope with 20x and 60x oil objectives in transmission mode equipped with either a PCO SensiCamQE camera, or a Phantom Miro (Vision Research) camera for higher temporal resolution movies were used. During recording the image is taken very slightly out of focus to enhance image contrast. High speed movies were recorded at 1000 frames per second with a 60x oil objective, 1.25 aperture, for a minimum of 7 seconds. Cell trajectories are derived from movies collected at $7 \mathrm{~Hz}$ using transmission light with a 20x objective from the center of mass using the track object feature of the Image-Pro Plus software from Media Cybernetics (see Fig.4.2).

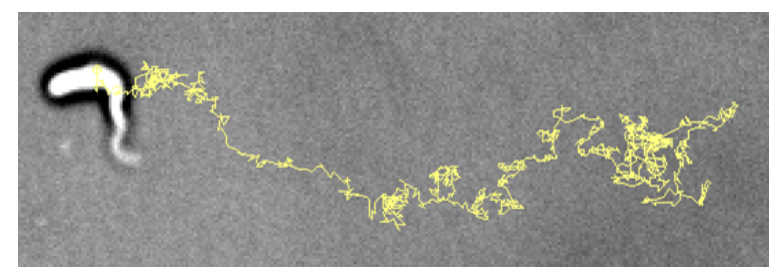

Figure 4.2: Typical track obtained from ImageProPlus track objects feature. Note that the image is taken slightly out of focus.

Using the xy coordinates derived from the trajectories, detailed statistical analysis was done using a combination of Matlab (Mathworks), and Origin (Origin Lab Corporation). All fitting was done using Origin with an exponential decay, $y=\exp \left(a_{0} / t_{p}\right)$

Characteristic time-lapse trajectories are shown given in Fig. 4.3 . The cells are in a homogeneous environment with no chemical gradients, therefore specific chemo-attraction may be ruled out as the basis for cell locomotion in this study. However, it is worth mentioning that although chemotaxis has not been demonstrated in trypanosomes, it is likely that they are capable of it [48]. 


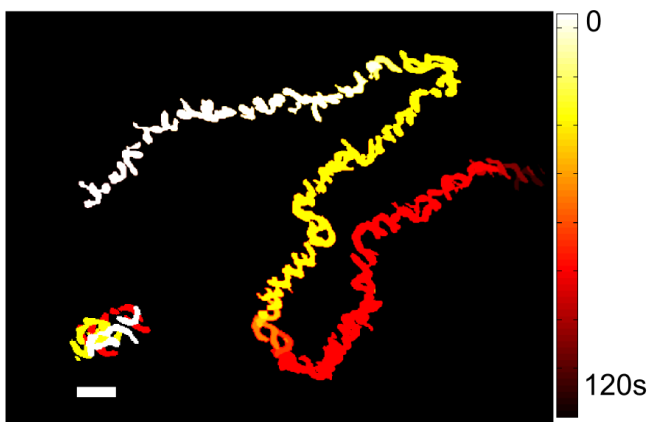

Figure 4.3: Time-lapse overlay of trypanosomes trajectory illustrates tumbling (lower left), and running motion (upper right), scale bar $20 \mu \mathrm{m}$, colorbar represents time, both trajectories represent about 2 minutes of trypanosome swimming.

\subsection{Results}

As seen in Fig. 4.3, trypanosomes from the same population taken from the same cell culture which are exposed to identical environmental conditions evidently do not follow a single motility mode. A deeper look at the trajectories revealed three motility modes, exemplified in Fig. 4.4 .
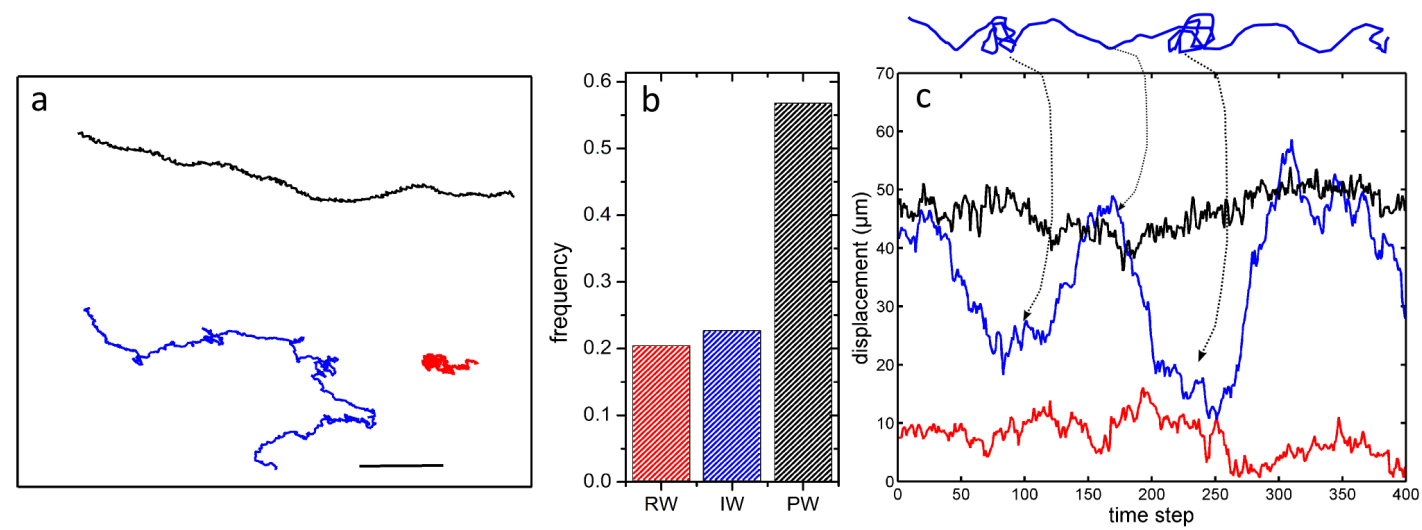

Figure 4.4: a. Diversity in trypanosome trajectories, reveals three motility modes in which cells tumble (random walk, RW in red), travel directionally (persistent, PW in black), or alternate between tumbling and running motion (intermediate, IW in blue). Scale bar 50 $\mu \mathrm{m}$. b. Distribution of motility modes. c. Displacement calculated between every 100 frames of recordings for each motility mode, with a corresponding schematic of a cell trajectory.

Of the trajectories that were recorded, about a quarter of the trypanosomes 'tumble' with no persistence in direction (shown in red in Fig. 4.4). These cells, referred to as random walkers (RW), do not seem to have a well defined orientation, hence the most frequently described anterior end-led swimming is not seen and little net displacement is observed. 
Secondly, about a half of the swimmers are highly directional - persistent walkers (PW) with a complete absence of tumbling motion within the observation interval. Cell orientation of these swimmers remains constant, with the anterior tip leading in the swimming direction.

The remaining population is comprised of cells that swim directionally with constant cell orientation but occasionally stop, tumble and reorient themselves and then move directionally again. Their trajectories resemble those of E.coli in which steady forward motion is interrupted by tumbling [24]. We refer to these cells as intermediate walkers (IW).
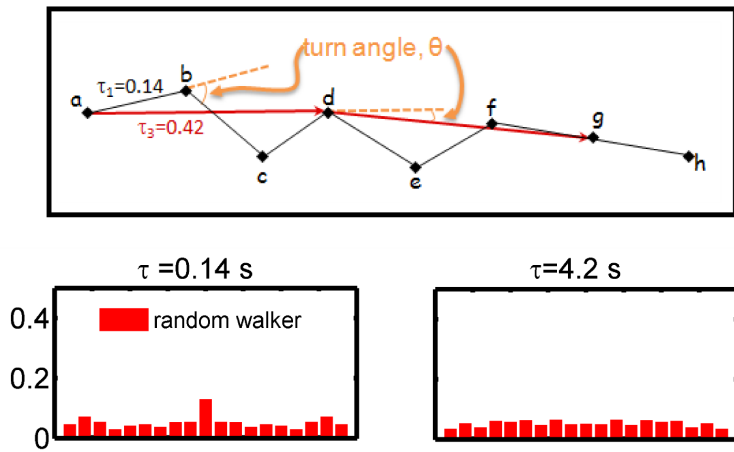

$\tau=4.2 \mathrm{~s}$
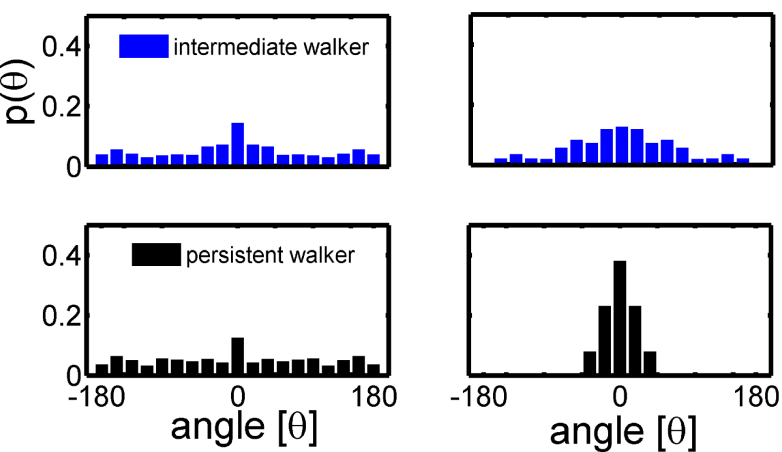

Figure 4.5: Turn angle $(\theta)$ distribution for RW, IW and PWs using the experimental sampling time 0.14s (left column) and 30x sampling time (right column). Sampling time $\tau$ is illustrated above. Differences in the turn angle distribution are apparent only when the turn angle is recorded at higher sampling times.

Fig. 4.4 clearly demonstrates that while RWs and PWs maintain low and high displacement values respectively throughout the observation interval, the IWs alternate or switch between the two 'states' thus warranting the classification of trypanosome motility into three distinct modes. Random walkers for which all turn angles have equal likelihood are characterized by a flat turn angle distribution, while individuals that are directionally persistent draw from a rather narrow turn angle distribution. We use the spread of the turn angle distributions $P(\theta)$, of each trajectory to systematically categorize indi- 
viduals into their respective swimming modes using empirically found thresholds for the spread of the turn angle distribution. Turn angle distributions for the trajectories shown in Fig. 4.4 are given in Fig. 4.5 illustrating the differences between motility modes.

Cell swimming was characterized using mean squared displacement (MSD) given by $\left\langle x(\tau)^{2}\right\rangle=\left\langle(r(t)-r(t-\tau))^{2}\right\rangle$, where $\left\langle x^{2}\right\rangle$ is the MSD, $r$ is position, and $\tau$ is the 'profile' time bound only by the time resolution of the experiment, typical curves are shown in Fig. 4.6.

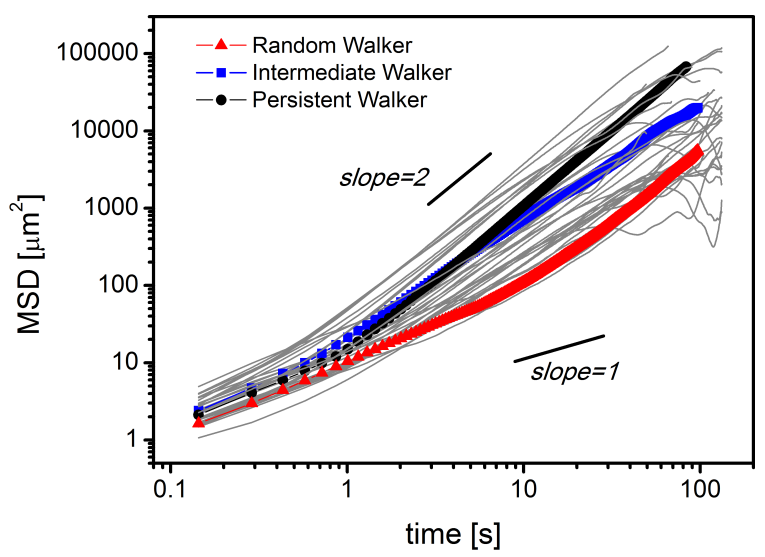

Figure 4.6: Mean squared displacement of typical swimmers for each mode .

Random walkers are characterized by an average scaling exponent near $\alpha \approx 1$, with $\left\langle x^{2}\right\rangle \sim 4 M t^{\alpha}$ where $M$ is the motility coefficient [38; 49] - indicating uncorrelated Brownianlike motion. The scaling exponent for persistent walkers tend to increase well above unity, indicating longer term correlations in swimming direction [38; 36]. For the intermediate walkers, scaling exponents are found to be in between those of the first two motility modes, see Table 4.1 for a summary.

Interestingly, as shown in Fig. 4.7 all three types of swimmers have virtually the same average swimming speed, demonstrating that these motility modes arise primarily due to directional motion.

The temporal decay of directionality is characterized by the cosine correlation function,

$$
\left\langle\frac{\mathbf{v}\left(t+t^{\prime}\right)}{\left|\mathbf{v}\left(t+t^{\prime}\right)\right|} \cdot \frac{\mathbf{v}(t)}{|\mathbf{v}(t)|}\right\rangle=\langle\cos \theta(t)\rangle
$$

where $\mathbf{v}$ is the velocity vector, $\theta$ is the angle between the two adjacent vectors separated by the time interval $t^{\prime}$, and \langle\rangle denotes an ensemble average. In the case of random walkers, the correlation decays rapidly and remains close to zero as shown in Fig. 4.8 .

For both persistent walkers and intermediate walkers a rapid decay in correlation is also seen, followed by a second slower decay. Sharp correlation drops for small time lags likely arise from the strong body distortions mentioned previously. An exponential fit of the average cosine correlation function for persistent walkers reveals a persistence time of $t_{p}^{\mathrm{PW}}=19.3 \mathrm{~s}$. For cells that alternate between tumbling and directional swimming, we find the average persistence time is significantly smaller, $t_{p}^{\mathrm{IW}}=12.4 \mathrm{~s}$. The mean tumbling 

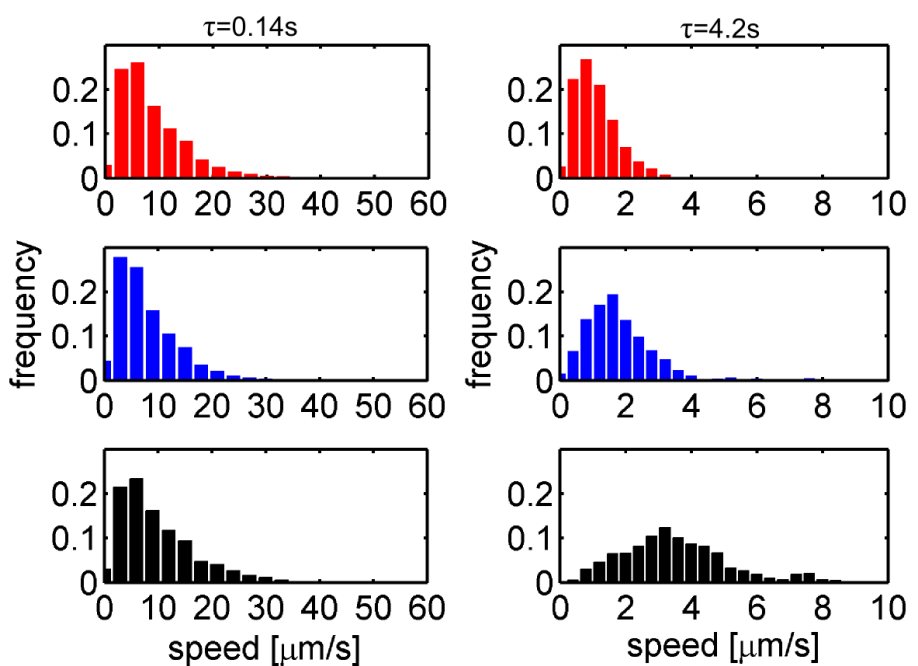

Figure 4.7: Population wide velocity distributions are virtually identical for all motility modes at experimental sampling time of $0.14 \mathrm{~s}$ (left column). At 30x sampling time (right column), the differences in motility modes are clear. Colour code as in Fig. 4.5

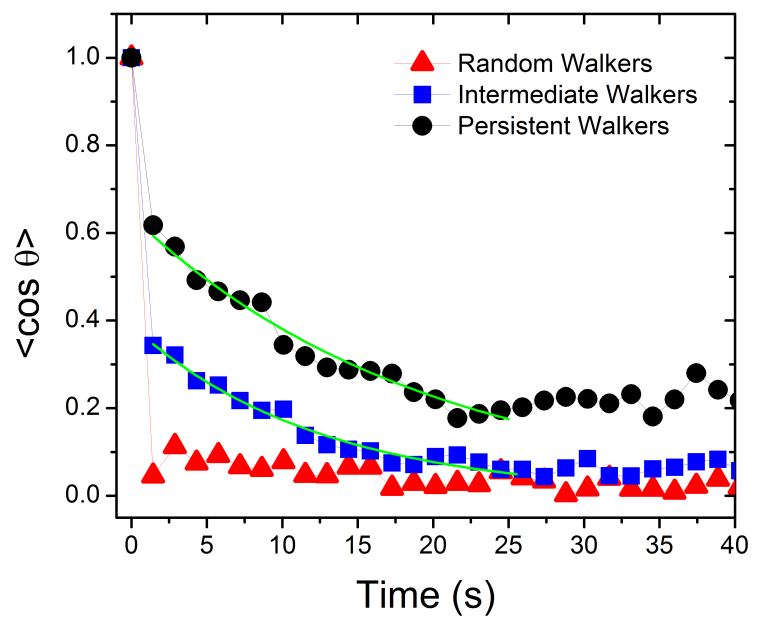

Figure 4.8: Average cosine correlation function for each motility mode, exponential fits for persistence time shown in green.

interval is $t_{t}^{\mathrm{IW}}=6.8 \mathrm{~s}$ - much longer than the tumble time of $0.1 \mathrm{~s}$ seen in E.coli[24]. Here a 'tumbling interval' was derived by the maximal time for which the distance traveled stays virtually constant with respect to time. Interestingly, all three correlations appear to remain above zero in the observation interval pointing to yet another longer term correlation. Although run and tumble behaviour has been characterized in E.coli for decades, the events that lead to these alternating modes are not understood in trypanosomes [27].

Table 4.1 summarizes the quantitative differences between the motility modes - highlighting the striking diversity in motility in a single trypanosome population. 
Table 4.1: Summary of trypanosome motility modes

\begin{tabular}{|c|c|c|c|}
\hline Swimming Mode & Random & Intermediate & Persistent \\
\hline Average Velocity $v(\mu \mathrm{m} / \mathrm{s})$ & 8.4 & 8.9 & 10.7 \\
\hline Persistence Time, $t_{p}(\mathrm{~s})$ & - & 12.4 & 19.3 \\
\hline Persistence Length, $L_{p}(\mu \mathrm{m})$ & - & 110.4 & 206.5 \\
\hline Average Scaling exponent, $\alpha$ (data) & 1.2 & 1.4 & 1.6 \\
\hline Scaling exponent, $\alpha$ (model) & 1.0 & 1.4 & 1.5 \\
\hline
\end{tabular}

The average velocity $v$ remains fairly constant in all motility modes, however the persistence time, $t_{p}$ reveals differences in motility modes arising from directional motion. The persistence length is given by $L_{p}=v t_{p}$. Differences in persistence are also represented in the scaling exponents obtained from power law fits of the MSD.

\section{Microscopic origin of motility modes}

In order to investigate the physical mechanisms for the observed motility behaviours, we examine trypanosomes at higher magnifications and at a much higher frame rate of $1000 \mathrm{~Hz}$. As mentioned in Chapter 2, very recent work with high speed microscopy has demonstrated surprisingly fast flagellar velocities up to 25 times faster than the average cell swimming speeds [31] further highlighting the need for high temporal resolution towards gaining a physical understanding of this parasite's motility.

The flagellum runs along the cell body resulting in complex body deformations during swimming, and while the quantitative descriptions of flagellar movements have been done for other species [50], little has been done for T. brucei brucei.

We choose a straightforward approach and examine the variations in the distance between the posterior end and the anterior end of the cell (referred to as end to end distance from this point) over time. Trypanosomes were tracked to ascertain their motility mode, and then followed by high speed recordings of their movement at a higher magnification. Recorded images were processed using median and Gaussian filters in the Matlab. As shown in the example in Fig. 4.9, a skeleton line through the center of the cell body was obtained.

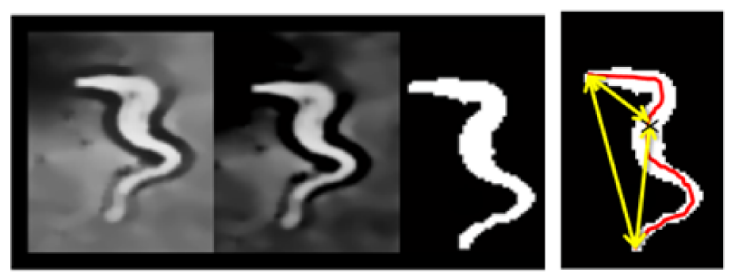

Figure 4.9: Image processing to obtain a skeleton line (red), and thereby extract the end to end distance. 
As indicated by the yellow arrow in Fig. 4.9 , for each frame the end to end distance was determined. We constructed a time series of end to end distances normalized to the length of the skeleton line [51; 52] which allows for comparison of motility modes as shown in Fig. 4.10.

a

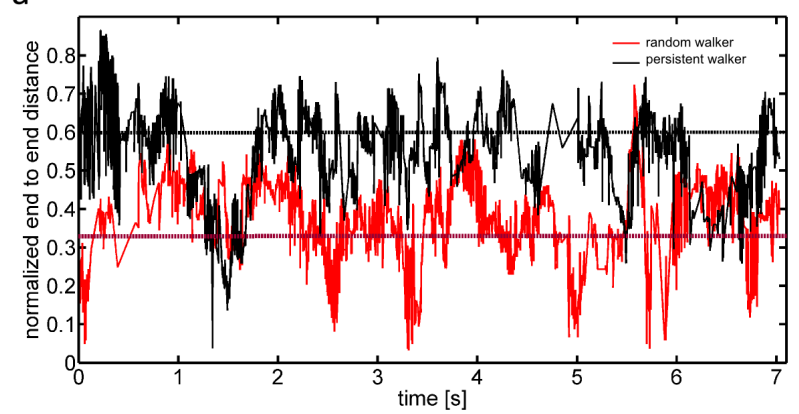

b

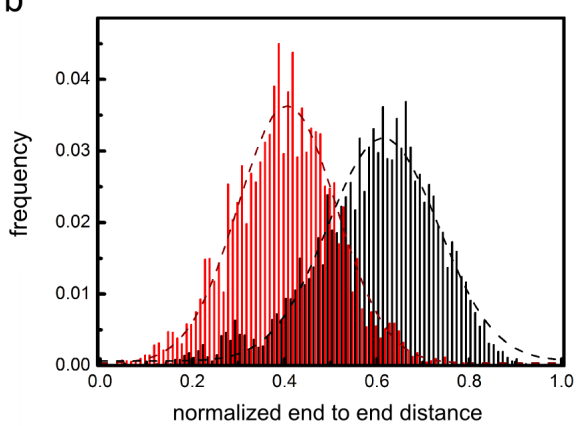

Figure 4.10: a. Two typical time series of end to end distance (normalized to cell length).b. Population distributions of end to end distance in persistent and random walkers

Histograms of the end to end distances show clear differences between the swimming behaviours (Fig. $4.10 \mathrm{p}$ ), and indicate that directional persistence could be the result of an elongated cell shape. Snapshots of the high speed movies of individuals demonstrate, as shown in Fig. 4.11, that cells swimming with directional persistence appear to take a more stretched body shape. Indeed the mean normalized end to end distance of persistent cells is 0.6 - more than 1.5 times that of random walkers for which it is 0.38 .

We ascribe the shape of the trypanosome to a worm-like chain with constant cell energy [51; 53]. The mean squared end to end distance $\left\langle R^{2}\right\rangle$ is given by $\left\langle R^{2}\right\rangle=2 \tilde{\kappa}^{2} L^{2}\left[e^{(-1 / \tilde{\kappa})}-\right.$ $1+1 / \tilde{\kappa}$ ] [54; 53]. Where $L$ is the length of the chain, and $\tilde{\kappa}$ is a dimensionless variable dependent on flexural rigidity, $\kappa$, and energy, $E$. Assuming equal energy utilization for self propelling motion in both motility modes, that is $E_{\mathrm{RW}}=E_{\mathrm{PW}}=E$, the ratio of the end

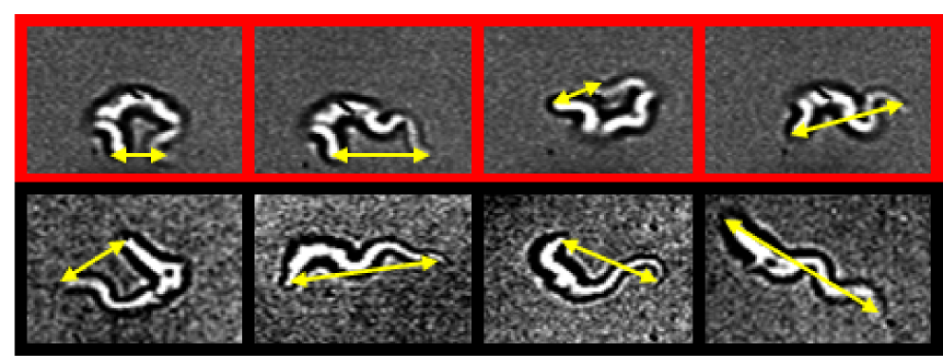

Figure 4.11: Snapshots from movies showing that persistent cells are indeed more stretched (black boxes) than random walkers (red boxes) 


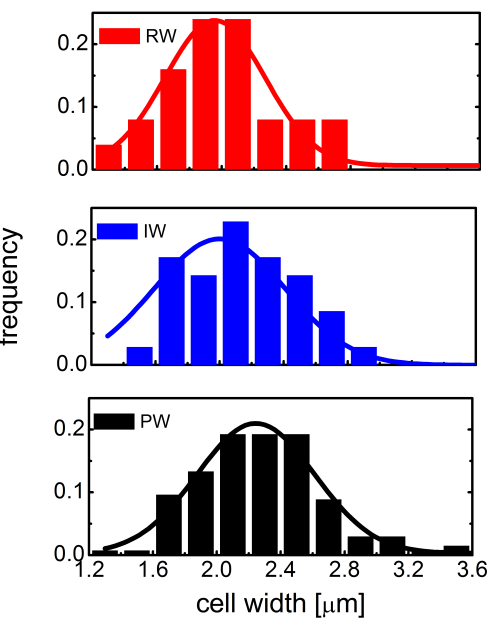

Figure 4.12: Distribution of cell width (measured at the widest point) for cells of each motility mode.

to end distances of the two motility modes is given by

$$
\frac{\left\langle R_{R W}^{2}\right\rangle}{\left\langle R_{P W}^{2}\right\rangle}=\frac{\kappa_{\mathrm{RW}}^{2}\left[e^{-1 / \kappa_{\mathrm{RW}}}-1+1 / \kappa_{\mathrm{RW}}\right]}{\kappa_{\mathrm{PW}}^{2}\left[e^{-1 / \kappa_{\mathrm{PW}}}-1+1 / \kappa_{\mathrm{PW}}\right]}
$$

Based on the above assumption of equal energy utilization, we find that persistent cells have three times more flexural rigidity than RW cells. The directional cells may be stiffer due to reorganization of motor proteins and crosslinking within the microtubules found both in the cell body and the flagellum. It is of course not unlikely that these observations are due to an interplay of differences in flexural rigidity and also energy utilization for cell motion which may depend on cell length [55].

These results are in qualitative agreement with theoretical work by Wada and Netz [17] on motility of the bacterium Spiroplasma in which softer cells were shown to flex more significantly due to random thermal fluctuations and thus were less efficient in directional motion. Spiroplasma has previously been reported to swim using a kinking helical mechanism [32] similar to the one recently suggested by Rodriguez et al. [31] for trypanosome swimming. Consistent motility modes have been identified in Spiroplasma [56]. Further, theoretical work suggests that changes in flexural rigidity of the cell have a direct effect on the pitch of the helical movement [57].

Cell division for blood stream form trypanosomes begins with the growth of a new flagellum. The cell body begins to expand while the kinetoplast, attached to the basal body, is replicated. Mitosis of the nucleus begins while the second flagellum continues to grow. Cell width may be used an indicator for the expansion associated with cell division. For this study, while only single cells that appeared to have a single flagellum were selected the exact stage of the cell cycle can only be assessed by measuring the cell width. In Fig. 4.12 the distribution of cell width (measured at the widest point) for cells in each motility mode is shown. On average, persistent walkers are wider than than TWs or 
IWs, indicating that they are already involved in cell division and the growth of a second flagellum. How cell motility is affected once cytokinesis progresses and daughter cells begin to resolve requires further investigation.

Aside from giving us clues about the microscopic origin of the observed motility modes, this data also allows us to study the dynamics of cell movement to further our understanding of the swimming mechanism of the trypanosomes. We track the movement of the posterior and anterior ends with respect to the center of mass of the cell, allowing us to isolate the whole body movement from the movement of the cell ends alone. Typical trajectories shown in Fig. 4.13 indicate that the anterior end of the cell tip appears to move faster than the posterior end of the cell.
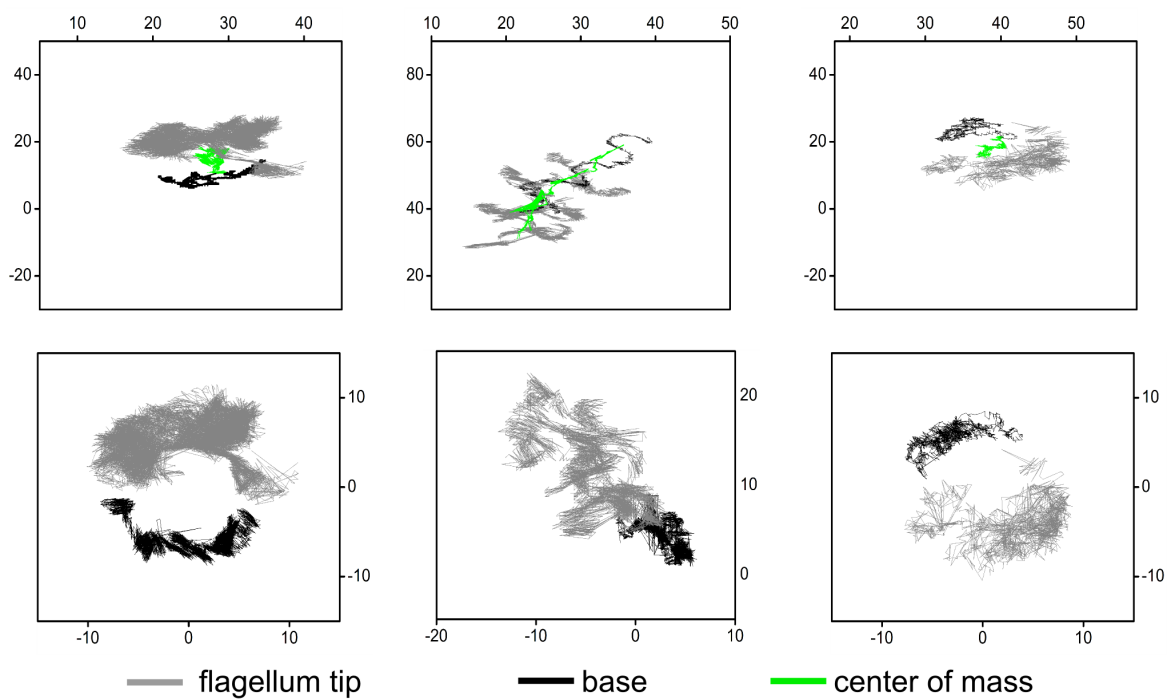

Figure 4.13: Trajectories of anterior (grey) and posterior (black) ends as well as center of mass (green) shown for $9 \mathrm{~s}$ (units are $\mu \mathrm{m}$ ). Top three panels show the movement of the anterior and posterior ends as well as the center of mass. Bottom panels show the corresponding cell end trajectories with a center of mass correction. Left and and right panels: persistent walkers, middle panel: random walker - persistence is not apparent at this time scale

Typical velocity distributions of cell ends, extracted from the trajectories of the anterior and posterior ends are shown in Fig. $4.14 \mathrm{a}$ and $\mathrm{b}$. We obtain mean anterior end (flagellar tip) velocities of $(546 \pm 143) \mu \mathrm{m} / \mathrm{s}$ and $(1060 \pm 400) \mu \mathrm{m} / \mathrm{s}$ for the random walker and persistent cells respectively while posterior end velocities are much lower for both RW and PW ((288 \pm 70$) \mu \mathrm{m} / \mathrm{s}$ and $(433 \pm 98) \mu \mathrm{m} / \mathrm{s}$ respectively) - all in fair agreement with [31]. This finding points to a 'velocity gradient' along the cell body, which is lowest at the posterior end and increases toward the anterior end. The gradient, which appears to be steeper in persistent walkers, in turn may stem from a gradual increase in elasticity due to the tapering body shape and from the bias in the hydrodynamic center of mass toward the posterior end. Changes in overall cell stiffness would hence not only affect flagellar 
velocities, but also the directionality in cell-end movement, thus determining the motility mode of the cell.
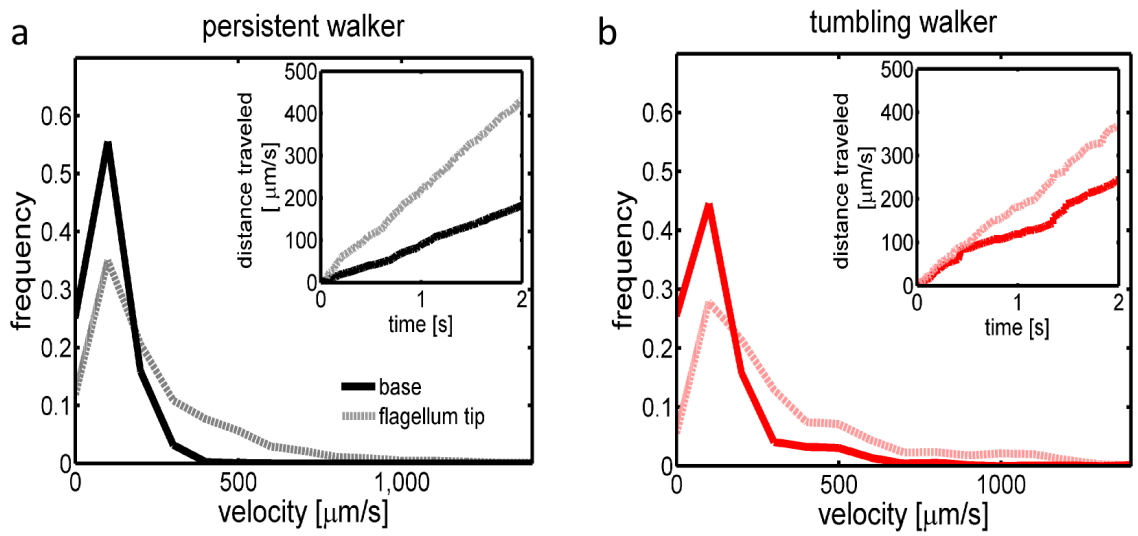

Figure 4.14: Typical velocity distributions for (a) persistent walker and (b) random walker (shown relative to center of mass), insets are distance traveled vs time for typical individuals - the slopes represent the velocity. In both swimming modes, the anterior (tip) end moves much faster than the posterior end (flagellar tip).

Notably, we find extremely fast cell body dynamics; the anterior end of the persistent walker moves over 50 times faster than the average whole cell swimming speed of 20 $\mu \mathrm{m} / \mathrm{s}$. Together these results support the notion that the motility modes are a direct consequence of differences in cell stiffness. The single pixel line to represent the cell body provides a straightforward method for further investigations into the movements of a cell and may shed light on the kinking mechanism recently suggested for trypanosome movement [31].

Finally, using a velocity autocorrelation function [58] for each cell end we are able to extract the fast time scales relevant to the cell movement. The correlation function is given by

$$
\mathbf{c}(i)=\sum \frac{(v(t)-\mu) \cdot(v(t+\tau)-\mu)}{\sigma^{2}}
$$

where velocity $v=\sqrt{\left(x_{i+1}-x_{i}\right)^{2}+\left(y_{i+1}-y_{i}\right)^{2}} / f, f$ is the temporal resolution, $\tau$ is the profile time or time lag, $\mu$ is the mean and $\sigma$ is the velocity standard deviation. In Fig. 4.15, a typical velocity autocorrelation function is shown for the posterior and anterior end of a cell.

An exponential fit to the correlation function reveals that the decay time is $(27 \pm 20)$ $\mathrm{ms}$ and $(82 \pm 10) \mathrm{ms}$ for the posterior and anterior ends respectively. These decay times do not appear to vary significantly across the three motility modes and point to surprisingly fast dynamics in cellular motility due to the fast body distortions. Note that the first fast decay is attributed mainly to resolution effects and therefore ignored in the fitting. Further, a longer periodicity of $\sim 1.2 \mathrm{~s}$ is seen in the autocorrelation functions (Fig. 4.15 
inset). This periodicity is likely to arise from the repeated 'bend and release' motions of the cell body which can clearly be seen in the trajectories shown in the middle panel of Fig. 4.13
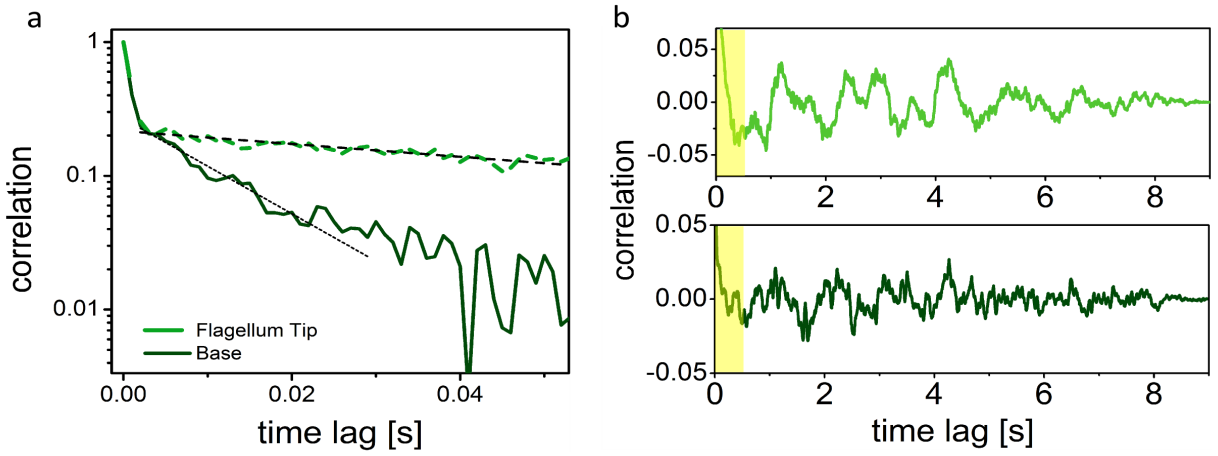

Figure 4.15: a. Typical velocity autocorrelation of cell ends. Both persistent and random walkers exhibit a faster decay in velocity autocorrelation for the posterior end than the anterior end (exponential fit - dashed black line). b. linear plot of correlation, shows a periodicity in velocity at $\sim 1.2 \mathrm{~s}$ (yellow shaded area is part a.)

\subsection{Modeling trypanosome swimming}

We outline here two models: a Pearson random walk model, and one with Langevin equations used to decipher the swimming trajectories studied above. The former distinguishes among the motility modes through the persistence time. The latter utilizes two decoupled Langevin equations for the average swimming direction and the rapid fluctuations in velocity consistent with experimental observations.

\subsubsection{Pearson random walk model ${ }^{1}$}

We describe a simulation model with which we are able to reconstruct the motion of a random walker and a persistent walker. A Pearson random walk model [24; 59; 37] is used where step size and turn angle are determined by two independent distributions, $P(\delta)$ and $P(\theta)$ respectively. The turn angle is determined by the previous displacement direction and a randomly drawn turn angle. Step sizes are drawn from an exponential distribution $P(\delta)=\lambda \exp (-\lambda \delta)$ with a characteristic displacement [59] $\langle\delta\rangle=1 / \lambda$ (equivalent for each motility mode). The model captures the motion of the trypanosomes, in particular their differences in persistence of translation motion see Fig. $4.4 \mathrm{~d}$ and Table 4.1 . However, it does not capture the smaller scale 'jaggedness' seen in the trajectories. This jaggedness arises from the rapid bending and twisting of the trypanosome cell body during swimming and is further discussed in the next section.

\footnotetext{
${ }^{1}$ in collaboration with Jan Nagler
} 
We consider a Pearson random walk [24; 59] with randomly drawn exponential distributed displacements $\delta$ and turn angles $\theta$. Let

$$
P(\delta)=\lambda e^{-\lambda \delta}
$$

the probability distribution function (pdf) for the displacements and

$$
P(\theta)=c_{N} e^{-\gamma|\theta|}
$$

the pdf for the turning angles where $-\pi \leq \theta \leq \pi$; $c_{N}$ being the normalization constant $c_{N}=\gamma\left(1-e^{-\pi \gamma}\right)$. Each time step $t=1,2, \ldots$, the displacement $r_{t}$ and the turn angle $\theta_{t}$ are chosen. Thereafter the $2 \mathrm{D}$ displacement vector is added to the actual position vector,

$$
\vec{r}_{t+1}=\vec{r}_{t}+\vec{r}_{t}
$$

where $\vec{r}_{t}=r_{t}\left[\cos \left(\theta_{t-1}+\theta_{t}\right), \sin \left(\theta_{t-1}+\theta_{t}\right)\right]$. It is interesting to note that first, any symmetric peaked shape of the turn angle pdf with well defined variance, can serve as a generating pdf for a Pearson random walk.

Second, for any $\gamma<\infty$ the Pearson walk becomes a normal random walk in the limiting case $t \rightarrow \infty$. Thus the mean-square displacement (MSD) is asymptotical linear in time, $\left\langle\vec{r}_{t}^{2}\right\rangle \sim t$. However, for intermediate time scales $t \approx 1 / \gamma$, the Pearson walker exhibits directional, or so-called persistent, motion, being an intermediate regime between normal diffusion $\left\langle\vec{r}_{t}^{2}\right\rangle \sim t(\gamma=0)$ and ballistic motion $\left\langle\vec{r}_{t}^{2}\right\rangle \sim t^{2}(\gamma=\infty)$.

The 3D direction correlation function, also called cosine correlation function, for symmetric displacement PDFs with finite variance, is given by

$$
C(t)=\langle\cos (\theta)\rangle
$$

where the turn angles $\theta$ are taken between successive displacements to a time scale $t$. For a $3 \mathrm{D}$ motion the correlation function can be derived as the mean cosine of the turning angles $c$ to the power of $t$ [59],

$$
C(t)=c^{t}
$$

We calculate the mean cosine of turning angles for their pdf, Eq.4.5, as

$$
c=\frac{\gamma^{2}}{1+\gamma^{2}} \operatorname{coth}(\pi \gamma / 2)
$$

where $\operatorname{coth}(x) \equiv\left(e^{x}+e^{-x}\right) /\left(e^{x}-e^{-x}\right)$. Finally, we readily obtain from Eq. 4.8 and Eq. 4.9 the $2 \mathrm{D}$ direction correlation function, given Eq. 4.5 , as

$$
C(t)=c^{t / 2}=e^{-t / t_{p}},
$$




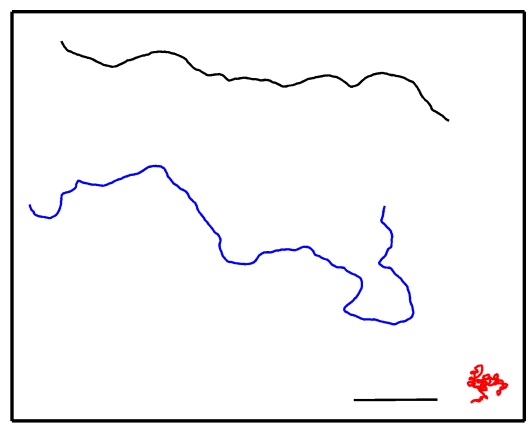

Figure 4.16: Trajectories generated by the Pearson random walk, compare to experimental trajectories in Fig. 4.4a. Scale bar $50 \mu \mathrm{m}$.

where

$$
t_{p}=-2 / \log (c)
$$

is the persistence time. Note that Eq. 4.10 is independent of the spatial scale $1 / \lambda$. Notably, for $\gamma=\infty$ the turn angle pdf becomes a delta function, and $C(t)=c=1$ whereas the normal random walk case $\gamma=0$ is represented in an uniform turn angle pdf implying a delta shaped correlation function $C(t)=\delta(t)$.

The 44 trypanosome trajectories display an exponential displacement distribution with mean value $\langle\delta\rangle=1.26 \mu \mathrm{m}$. For the model we therefore assume the overall displacement distribution $P(\delta)=\lambda \exp (-\lambda \delta)$ with $\lambda=1 / \delta$. We plugged the fitted values for the persistence times $t_{p}^{\mathrm{IW}}$, for intermediate walkers, and $t_{p}^{\mathrm{PW}}$, for persistent walkers (Table 4.1 in the results section), into Eq. 4.11. As explained above, the persistence time for the random walker class $t_{p}^{\mathrm{RW}}$ is heavily determined by the fast rotation motion. Here we use, however, the fitted value $t_{p}^{\mathrm{RW}} \approx 0.60 s$ for illustration. Finally, for the three motility modes, solving Eq. 4.11 for $\gamma$ yields $\gamma_{\mathrm{RW}}=1.21, \gamma_{\mathrm{IW}}=6.55$, and $\gamma_{\mathrm{PW}}=8.19$, respectively. Complementary to Fig. 4.4 and Table 4.1 in the main text, we exhibit in Fig. 4.16 trajectories produced from this above described model. We see that these trajectories bear close resemblance to those shown in Fig. 4.4 for all three motility modes.

\subsubsection{Langevin equations ${ }^{2}$}

While the Pearson model could fully describe the three motility modes, it does not account for the zig zag pattern (arising from rapid body fluctuations) of the trajectories seen in Fig. 4.4. Langevin equations have been employed in the past to describe motility patterns of microorganisms [36; 60].

We start our analysis with the time series of the trypanosomes' center of mass velocity

${ }^{2}$ in collaboration with Vasily Zaburdaev 
defined for each time step $t_{i}$ as

$$
\mathbf{v}\left(t_{i}\right)=\left[\mathbf{r}\left(t_{i}+\delta t\right)-\mathbf{r}\left(t_{i}\right)\right] / \delta t
$$

Clearly the time series depend on the choice of $\delta t$. For now we set $\delta t=2 \mathrm{~s}$ and comment on this choice later. As seen in Fig. 4.8, the direction of motion exhibits two characteristic relaxation times that differ by an order of magnitude indicating that the speed and direction of motion of trypanosomes can be decoupled on time scales exceeding $1 \mathrm{~s}$ [61].

The distribution of speed values for all trajectories exhibits a broad non-Gaussian profile (see Fig. 4.7). Non-Gaussian velocity distributions have been reported and explained for other types of microorganisms [36; 60; 62].

In our case the reason for the broad distribution can be attributed to the fact that the cells are not identical (as discussed above) and each trypanosome trajectory can be characterized by its mean square velocity $\left\langle\mathbf{v}^{2}\right\rangle$, where $\langle\ldots\rangle$ signifies average over one trajectory.

The differences between each individual, are characterized by the fluctuations around the mean squared velocity. For each trajectory we calculate the standard deviation of velocity squared, $\delta \mathbf{v}^{2}=\left\langle\left(\mathbf{v}^{2}-\left\langle\mathbf{v}^{2}\right\rangle\right)^{2}\right\rangle^{1 / 2}$, and the average directional "persistence" as in Eq. $4.1(\delta t=2 \mathrm{~s})$, and then check how they depend on the trajectory's mean square velocity. Faster trypanosomes display larger velocity fluctuations $\delta \mathbf{v}^{2}$ as the approximately quadratic dependence on $\left\langle\mathbf{v}^{2}\right\rangle^{1 / 2}$ shows, but their trajectories are more straight, since consecutive velocity directions are more aligned (see Fig. 4.17). These two facts indicate that the amplitude of fluctuations linearly depends on the speed and also that the increasing speed makes the cells more persistent.

We now formulate a model which accounts for all of the aforementioned observations using Langevin equations. The body deformations of the trypanosome on a time scale of less than $1 \mathrm{~s}$ are the primary source of fluctuations and correlations of the average swimming direction decay more slowly. To capture this behaviour, we split the velocity of the trypanosome into two parts: $\mathbf{v}=\mathbf{w}+\mathbf{u}$.

The velocity fluctuations are described by $\mathbf{w}$ while the component $\mathbf{u}=u_{0}(\cos \varphi, \sin \varphi)$ with the constant swimming speed $u_{0}$ and slow changes in direction through the polar angle $\varphi$. We describe the time evolution of the two dynamic variables, $\mathbf{w}$ and $\varphi$, with the help of Langevin equations.

The polar angle $\varphi$ diffuses on the unit circle and, therefore, obeys $\dot{\varphi}=g_{\varphi} \eta(t)$. Here $\eta(t)$ is standard delta-correlated additive white noise with zero mean and variance $\left\langle\eta(t) \eta\left(t^{\prime}\right)\right\rangle=$ $\delta\left(t-t^{\prime}\right)$. The noise amplitude $g_{\varphi}$ determines the longest correlation time $\tau_{\varphi}=2 / g_{\varphi}^{2} \sim 10 \mathrm{~s}$ visible in Fig. 4.8. Note that this value clearly indicates that the rotational diffusion of the average swimming direction is a result of active rather than thermal motion of the trypanosome. This becomes clear when we estimate the corresponding thermal relaxation time $\tau_{\text {th }}$ at room temperature in water by assuming that the trypanosome is a hard rod of length $L=20 \mu \mathrm{m}$ and diameter $d=2 \mu \mathrm{m}$, we arrive at $\tau_{\text {th }} \simeq 1000 \mathrm{~s}$, much larger than $\tau_{\varphi}$. 
a

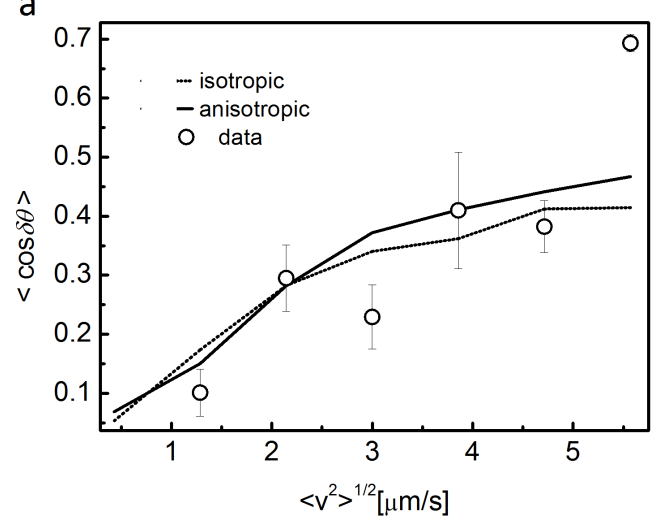

$b$

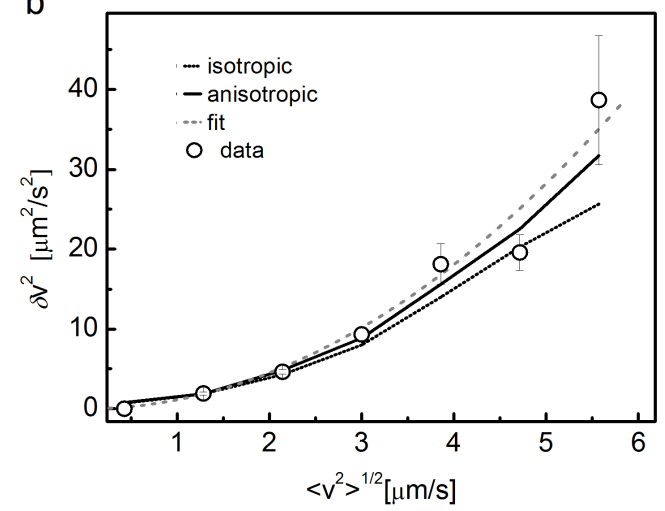

Figure 4.17: Experimental data is compared to simulated data from a model with isotropic (dashed line) and anisotropic noise (solid line). a. Directional "persistence" of a trajectory grows with the mean velocity of a single cell. b. Velocity fluctuations $\delta \mathbf{v}^{2}$ as a function of the root mean square velocity $\left\langle\mathbf{v}^{2}\right\rangle^{1 / 2}$ of an individual cell.

The fast velocity fluctuations $\mathbf{w}$ are described by a standard Langevin equation [63]:

$$
\dot{\mathbf{w}}=-\mathbf{w} / \tau_{w}+g_{w} \boldsymbol{\xi}(t) / \sqrt{\tau_{w}},
$$

where $\boldsymbol{\xi}(t)=\left(\xi_{1}, \xi_{2}\right)$ is a two-dimensional vector with independent white noise components, $\left\langle\xi_{i}(t) \xi_{j}\left(t^{\prime}\right)\right\rangle=\delta_{i j} \delta\left(t-t^{\prime}\right)$, and $g_{w}$ defines the strength of noise. Our experimental data also shows that faster cells exhibit larger velocity fluctuations. Therefore, we assume that the strength of the noise is a linear function in the cell's average speed $u_{0}$ : $g_{w}\left(u_{0}\right)=g_{0}+\alpha u_{0}$. With recorded swimming speeds $\lesssim 15 \mu \mathrm{m} / \mathrm{s}$, the trypanosome is a low Reynolds number swimmer $\left(\operatorname{Re}<10^{-4}\right)$ where inertia is completely negligible [15]. However, Langevin Eq. 4.13 conventionally describes the thermal diffusion of a Brownian particle with inertia. In our case, the inertial term and also the athermal noise are due to the active cell motion (we elaborate on the swimming mechanism below). As such, the present Langevin equation provides the short relaxation time $\tau_{w}$ in the trypanosome's velocity correlations and is standard in the stochastic analysis of moving microorganisms (see the discussion in [36]).

The speed $u_{0}$ characterizes a single trypanosome. For each trajectory $\left\langle\mathbf{v}^{2}\right\rangle=u_{0}^{2}+\left\langle\mathbf{w}^{2}\right\rangle$, where $\left\langle\mathbf{w}^{2}\right\rangle=\left(g_{0}+\alpha u_{0}\right)^{2}$, which allows us to solve for $u_{0}$ for each value of $\left\langle\mathbf{v}^{2}\right\rangle$. However, the time step $\delta t$ used in Eq. 4.12 for calculating velocity from experimental data, is larger than the short relaxation time $\tau_{w}$ in Eq. 4.13 . Consequently, the experimental value $\left\langle\mathbf{w}^{2}\right\rangle_{\exp }$ is smaller than the ideal or instantaneous value $\left\langle\mathbf{w}^{2}\right\rangle=\left(g_{0}+\alpha u_{0}\right)^{2}$ predicted by theory. We are able to link both values in the following relation [64]:

$$
\left\langle\mathbf{v}^{2}\right\rangle_{\exp }=u_{0}^{2}+\left\langle\mathbf{w}^{2}\right\rangle \frac{2 \tau_{w}}{\delta t}\left(1-\frac{\tau_{w}}{\delta t}\left[1-e^{-\delta t / \tau_{w}}\right]\right) \text {. }
$$


This recovers the ideal relation in the limit $\delta t \ll \tau_{w}$. We use Eq. 4.14 to determine the distribution of $u_{0}$ from the experimental $R\left(\left\langle\mathbf{v}^{2}\right\rangle^{1 / 2}\right)$.

Finally, we found that the average speed of cells slowly decreases in time for cell trajectories recorded for up to $30 \mathrm{~min}$, likely due to the illumination from the microscope lamp. The characteristic time of the slow evolution $(\sim 20 \mathrm{~min})$ is an order of magnitude larger than the window of interest. Nevertheless, to complete our model, we take into account the aging of the cells due to illumination by introducing exponential decay laws $u_{0}(t):=u_{0} e^{-t / T}$ and $g_{w}(t):=g_{w} e^{-t / T}$ with $T=1100 \mathrm{~s}$.

Theoretical modeling vs. experiment: Based on our model, we use computer simulations [65] to generate numerical data for the velocity time series. By finding the best match between the numerical and experimental results for the autocorrelation functions, velocity distribution, and the amplitude of velocity fluctuations, we determine the four unknown parameters of our model: the noise amplitudes $g_{\varphi}$ and $g_{w}=g_{0}+\alpha u_{0}$, and the fast relaxation time $\tau_{w}$. A successful model should not depend on the choice of the time step $\delta t$ used in the definition of velocity (Eq. 4.12). Indeed, simulated and experimental data could be matched with the same parameters for various time steps $\delta t=1,2$, and $3 \mathrm{~s}$.

The agreement between simulations and experiments is excellent, as documented in Fig. 4.17. The dashed lines in Fig. 4.17 of our current model indicate that with increasing $\left\langle\mathbf{v}^{2}\right\rangle$ the faster trajectories become straighter and fluctuations $\delta \mathbf{v}^{2}$ grow.

To further advance the model, we introduce anisotropic noise in Langevin equation (Eq. 4.13) by distinguishing between the respective noise components parallel and perpendicular to the average swimming direction $\mathbf{u}, g_{\|}=g_{0}+\alpha u_{0}$ and $g_{\perp}=g_{0}+\alpha \gamma u_{0}$. The coefficient $\gamma$ characterizes the anisotropy of noise. As we have seen above, the elongation of the cell body is related to its stiffness and that stiffer cells are faster and more persistent. Therefore, we expect fluctuations orthogonal to the body to be weaker, meaning that $\gamma \in[0,1]$. Indeed, both fits in Fig. 4.17 are improved by the anistropic noise.

The fit parameters of our model assume the following values: $\tau_{w}=0.3 \mathrm{~s}$ and $\tau_{\varphi}=$ $2 / g_{\varphi}^{2}=11.3 \mathrm{~s}$; for the isotropic noise, $g_{0}=1.5 \mu \mathrm{m} / \mathrm{s}$ and $\alpha=1.8$, while for the anisotropic noise, $g_{0}=1.8 \mu \mathrm{m} / \mathrm{s}, \alpha=1.9$, and $\gamma=0.45$ indicates a pronounced anisotropy. Note that our model is not only consistent with experimental data, but also provides a prediction for the short relaxation time $\tau_{w}=0.3 \mathrm{~s}$, beyond the experimental resolution of $1 \mathrm{~s}$. Note that 22 trajectories with $1 \mathrm{kHz}$ resolution showed shortest correlation time to be $0.12 \mathrm{~s}$ (Fig. 4.18).

Combining our findings with previous work we arrive at the following physical picture of swimming trypanosomes. There is a distortional wave [31] that runs from the thinner to the thicker end of the elongated cell body. It creates the non-reciprocal body deformation necessary for locomotion at low Reynolds numbers [15]. The wave produces large amplitude oscillations of the thin end of the cell, similar to a recently constructed microswimmer [4], which results in the observed zig-zag motion with angular amplitude $\delta \varphi$ around the average swimming direction $\mathbf{u}$. However, the oscillations are irregular and 


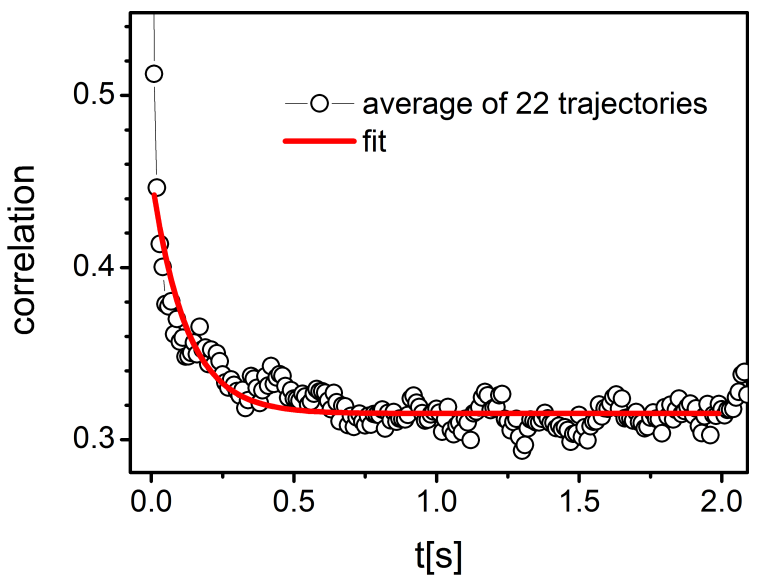

Figure 4.18: Average speed autocorrelation given by Eq. 4.3 for the center of mass of 10 trajectories. Relaxation time is extracted from the exponential fit shown in red.

correlations in the rapid cell-body motion decay within $\tau_{w}=0.3 \mathrm{~s}$. This time corresponds to a few oscillations and is consistent with the reported flagellum beating period of $50 \mathrm{~ms}$ [31]. During $\tau_{w}$, u performs an angular random walk step $\delta \varphi$. Then, temporal correlations in $\mathbf{u}$ vanish after time $t_{\varphi}$ meaning $\pi^{2} \simeq \delta \varphi^{2} t_{\varphi} / \tau_{w}$. This gives a reasonable estimate $\delta \varphi \simeq \pi / 6$ and links $t_{\varphi}$ to characteristics of the rapid cell motion.

We modeled the trypanosome's swimming path using two decoupled Langevin equations for the average swimming direction and the rapid fluctuations in velocity consistent with experimental observations. The stochastic forces involved are athermal and a result of the actively moving trypanosome. Properties of individual cells vary strongly, with faster cells having larger velocity fluctuations and straighter trajectories. The second observation motivated an anisotropic extension of our model with stronger velocity fluctuations along the swimming direction that improved the modeling.

The theoretical model explains the intriguing features of trypanosome motility and quantitatively describes the large-scale motion of the trypanosome but also takes into account its distinct body distortions.

\subsection{Summary}

Although trypanosome run and tumble motion has been reported previously [66], different motility modes in T. brucei brucei had never been characterized. Directionality may confer the ability to invade into tissue - the last stage of sleeping sickness is characterized by parasite invasion of the blood brain barrier [67], or it may be an effective nutrient search strategy[60]. On the other hand, cell swimming has been shown to be essential for host antibody removal and one of these motility modes may increase local hydrodynamic drag on surface proteins [5]. We have been able to show that the macroscopic motility modes are a result of varying cell stiffness. Our analysis of cell end to end dis- 
tance provides a rapid screen for identification of differences in microscopic properties of cells. Finally our analysis points to remarkably fast cell motility dynamics influencing both the intrinsic rotational and translational cellular motion. The Pearson walk model accounts for the differences in motility modes, primarily through differences in directionality. Further, a model based on Langevin equations could account for the the fast body undulations for the movements of the cell body. 



\section{Motility in tunable environments}

Blood stream form trypanosomes are confronted with a wide range of environmental conditions. Besides blood vessels, they also penetrate the extracellular matrix (ECM) of tissue spaces. A specific occurrence of this is when they penetrate the blood brain barrier (BBB) during the second stage of Sleeping Sickness and enter the central nervous system (CNS). The BBB is comprised of a layer endothelial cells bound by tight junctions, and lined with blood capillaries. In the so-called 'immuno-privileged' organs of the CNS, the parasite is safe not only from the host immune system, but also from most medical drugs. How then do trypanosomes tunnel their way through such barriers without the use of any known chemical agents to break them down? A fundamental knowledge of how these parasites swim within such an environments is needed to answer these questions. Though the exact nature and composition of the ECM depends on the type and location of the tissue in the body, it is primarily composed of collagen, various polysaccharides, proteoglycans, and other adhesive proteins that bind to specific cell surface receptors. Together these components form a dense network that serves to provide strength, adhesion, and filtration (Fig. 5.1).

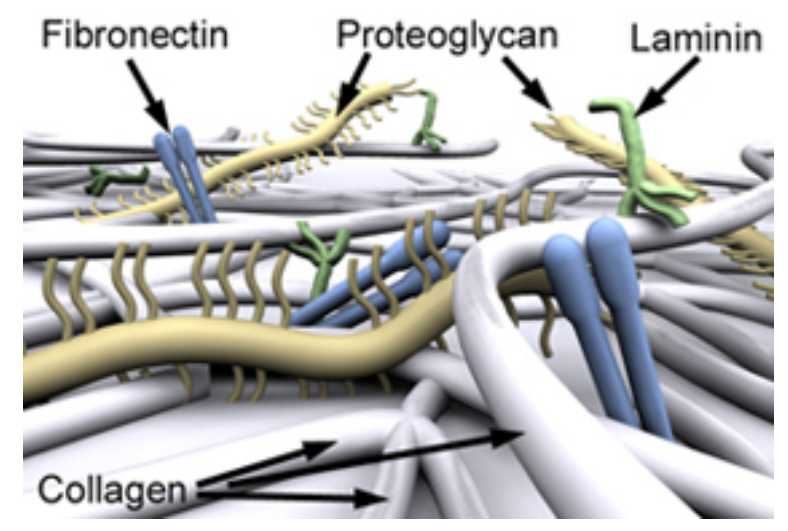

Figure 5.1: The extracellular matrix is a complex network of fibers and other structural proteins, from [68].

Collagen matrices formed in vitro are cross linked and used as a model system to study cell behaviour in a tissue like environment due to the close resemblance in mechan- 
ical features [69: 70]. Having studied trypanosome motility in a minimal environment in the previous chapter, here we examine the behaviour of trypanosomes in in vitro collagen networks as well purely physical networks of tunable mesh size using F-actin. Finally viscosity is manipulated using dextran. We further explore the use of trypanosomes as active probes to extract network properties.

\subsection{Experiment}

As in Chapter 4, single cell trajectories are observed between glass slides, however the surrounding medium in which the trypanosome cells are suspended is varied. Data were collected at $7 \mathrm{~Hz}$ to ensure uniformity with the trajectories of Chapter 4 collected in culture medium.

\subsubsection{Dextran preparation}

The viscosity of the solution can be manipulated by changing the concentration of dextran in culture medium. Dextran is a complex branched polysaccharide composed of many glucose molecules. Dextran was purchased from Sigma Aldrich (Germany) and used in $5 \%, 8 \%$ and $25 \% \mathrm{w} / \mathrm{v}$ concentrations in HMI9 medium as the suspending solution. The measured viscosities were $3.5 \mathrm{cP}, 5.45 \mathrm{cP}$, (as measured by a Ubbelohde viscometer) and $\sim 30 \mathrm{cP}$ [71] respectively. Dextran solutions up to $30 \% \mathrm{w} / \mathrm{v}$ in concentration are Newtonian liquids [72] meaning that viscosity does not change as flow is induced.

\subsubsection{Actin preparation}

Actin is a highly conserved cytoskeletal protein found in almost all eukaryotic cells. It has many roles within a cell including mechanical support, motility, force generation, cargo transfer mediation, cytokinesis, and cell junctions. Globular actin monomers (Gactin), with a molecular weight of $42 \mathrm{kDa}$ are comprised of an ATP binding cleft that is stabilized against denaturation by the binding of ATP. A two-stranded helical filament (F-actin) with a diameter of about $8 \mathrm{~nm}$ and a helical pitch of $37 \mathrm{~nm}$ is assembled from Gactin. In vitro polymerization can be initiated by the addition of salts. The process of actin polymerization in vitro can be divided into three steps (see Fig. 5.2). A stable aggregation of three to four actin monomers leads to nucleation which in turn allows for a growth phase in which ATP-G-actin is successively added to the existing filament. The ATP can be slowly hydrolyzed to ADP (adenosine diphosphate) and stable ADP-F-actin leading to disassembly.

Lyophilized rhodamine labeled and unlabeled G-actin from rabbit muscle (purchased from Cytoskeleton, Denver, USA; purity $>99 \%$ ) is stored at $-70^{\circ} \mathrm{C}$, and thawed on ice. The powder is dissolved in ultrapure water (produced by a Milli-Q facility, Millipore, Germany) to $0.2 \mathrm{mg} / \mathrm{mL}$ and $1.2 \mathrm{mg} / \mathrm{mL}$ respectively. Labeled and unlabeled actin were 


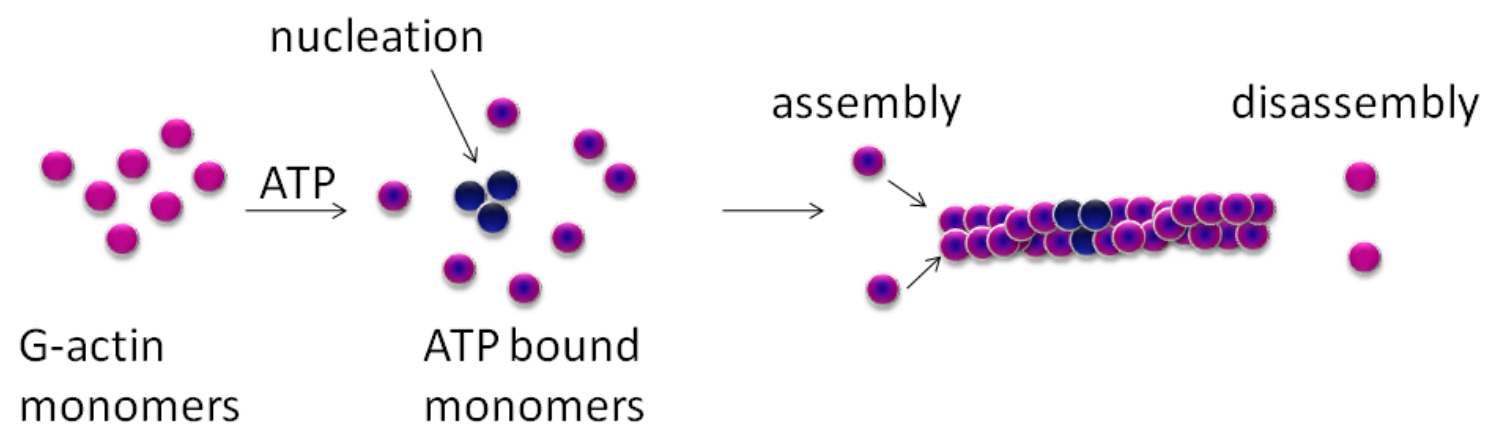

Figure 5.2: Actin polymerization is a three step process including nucleation, growth phase, and steady-state. Stable nuclei are elongated by the addition of ATP-G-actin. Stable ADP-F-actin results from ATP hydrolysis. The system is in steady-state when the rate of ATP-G-actin addition equals the rate of ADP-G-actin loss.

mixed in a 1:1 ratio. The G-actin solution is diluted in MonoMix (lyophilized G-actin ATP-buffer) to the desired concentration and kept on ice for two hours to allow for depolymerization of existing oligomers. This step reduces the number of polymerization nuclei and thus leads to fewer but longer filaments. PolyMix (lyophilized F-actin ATPbuffer, $\mathrm{pH}$ 7.4) is added and the solution is incubated at room temperature for two hours (volume ratio A-buffer : polymerization buffer $=9: 1$ ). The solution is thoroughly mixed and kept at $4^{\circ} \mathrm{C}$ until it is used for the experiments. Polymerized actin can be kept at $4^{\circ} \mathrm{C}$ for several weeks without observable degradation of the filaments. Rhodamine is excited at a wavelength of $\lambda_{e x}=552 \mathrm{~nm}$ and its emission wavelength is $\lambda_{e m}=575 \mathrm{~nm}$.

The polymerized actin is well mixed and combined with a cell suspension to the desired actin concentration. Actin provides a fairly homogeneous physical network with minimal chemical cross-links and is therefore a model for the study of trypanosome motility in polymer networks similar to the extracellular matrix. We tested a range of actin concentrations from $0.01 \mathrm{mg} / \mathrm{mL}$ to $0.5 \mathrm{mg} / \mathrm{mL}$ dissolved in culture medium. At even higher concentrations, the trypanosomes seemed to exhibit impaired motility likely due to reduced nutrient availability.

\subsubsection{Collagen preparation}

Collagen is the major component of fibrous proteins in the ECM. More than 20 different types of collagen, representing about $25 \% \mathrm{w} / \mathrm{v}$ of the total protein mass in animals, have been identified and classified primarily according to their physiological structure. Network-forming collagens assemble into a feltlike sheet or meshwork. Here, we use collagen I, which represents $90 \%$ of the total body collagen and can easily be isolated from tendon-rich tissue such as rat tails. 


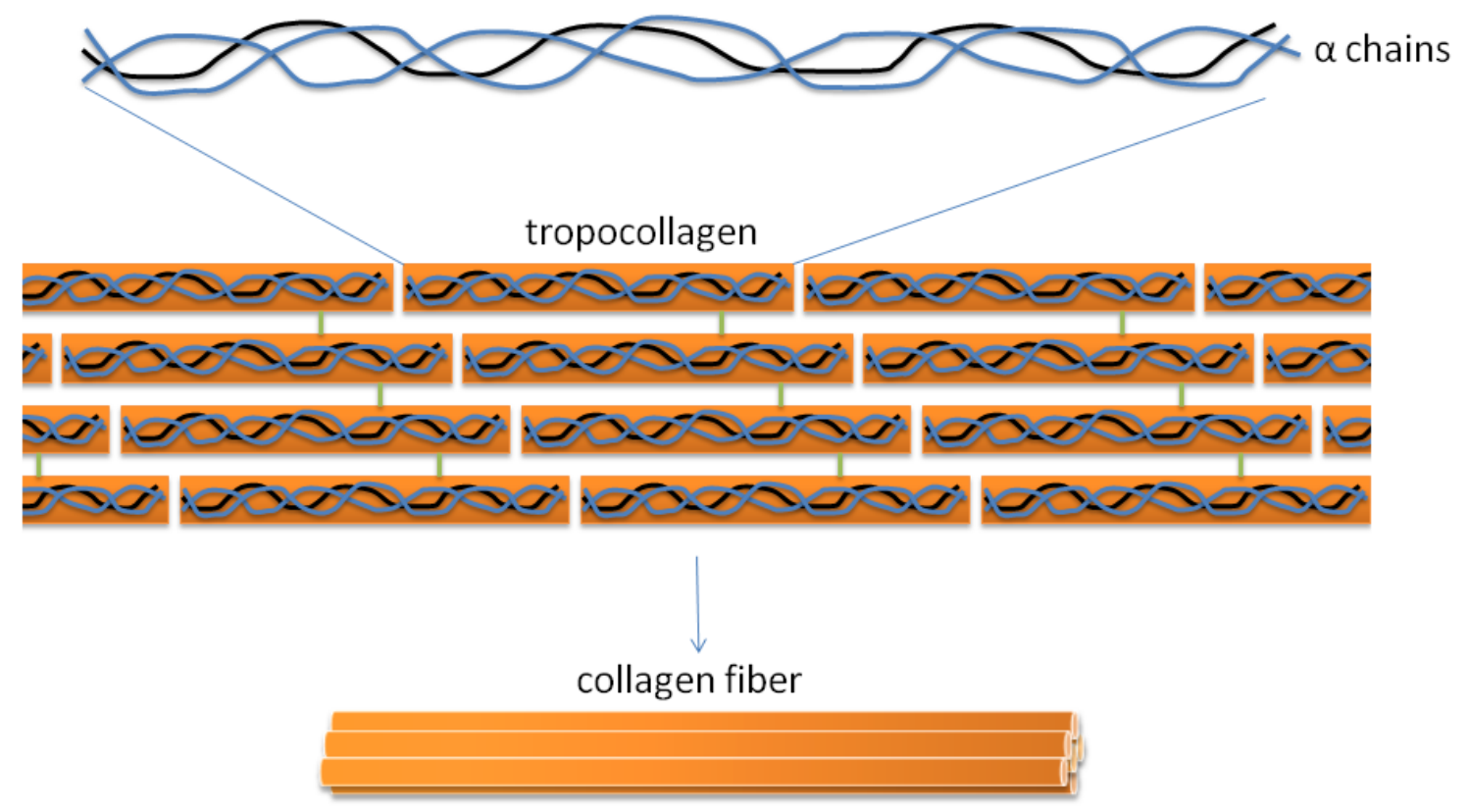

Figure 5.3: Collagen fiber assembly.

The fundamental unit of collagen is a $300 \mathrm{~nm}$ long, $1.5 \mathrm{~nm}$ thin wide protein comprised of two $\alpha 1$ chains and one $\alpha 2$ chain. These three subunits are wound around each other as shown in Fig. 5.3. Many such triplet coils join side by side in a staggered manner through covalent bonds to form fibrils that are 50-200 nm in diameter. In the extracellular space, the collagen molecules hierarchically self-assemble at the nano-, micro-, and macroscales into many different structures which are adapted to their physiological function.

Collagen structure is of prime importance in development and disease. Collagen extraction was initially hindered due to its insolubility. However, it was found later that tropocollagen from young animals was cross linked to a lesser extent and thus allowed for in vitro studies. We now study trypanosome motility in in vitro of collagen networks. In vitro, collagen gels are easily formed through controlled changes of $\mathrm{pH}$ and temperature. Here we use rat tail collagen type I (BD Biosciences, Germany) by bringing the $\mathrm{pH}$ to alkalinity using $1 \mathrm{~N} \mathrm{NaOH}$ as per the manufacturer's instructions. Fig. 5.4 exemplifies the gels that can be formed at various concentrations of collagen with trypanosomes.

Clearly collagen fibers are thicker than actin fibers allowing for direct observation under bright field transmission microscopy. Despite thorough mixing before placement on a microscope slide, the collagen network is not as homogeneous as the actin network. 

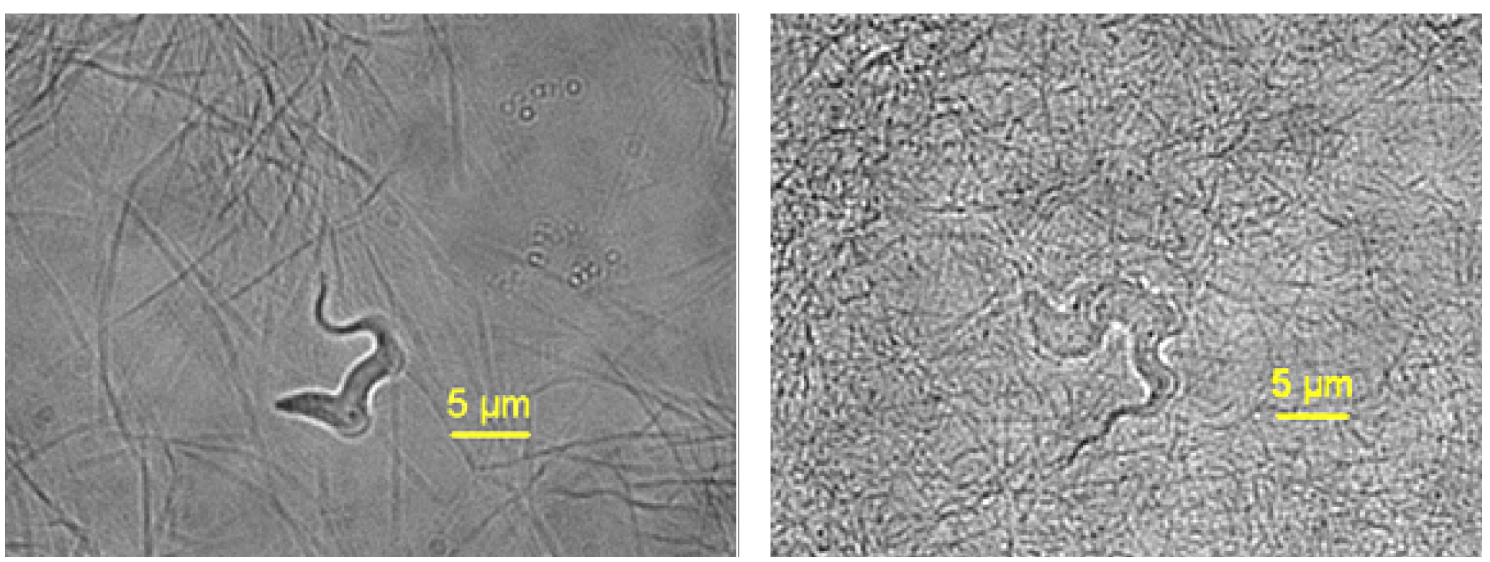

Figure 5.4: Collagen networks at various concentrations with trypanosomes embedded.

\subsection{Results}

We observe individual isolated cells in between two glass slides in a quasi 2D environment as in Chapter 4 in the tunable environments just described. Care was taken to ensure that the distance between the glass slides $(\sim 10 \mu \mathrm{m})$ allowed for sufficient movement of the trypanosomes through the network and not above or below it directly adjacent to the glass slides.

\subsubsection{Actin}

Between 10 and 30 cells in a range of actin concentrations between 0 and $0.5 \mathrm{mg} / \mathrm{mL}$ networks were observed and their trajectories were recorded and extracted as reported in Chapter 4. In Fig. 5.5, the distance traveled by individual cells over time, $d(t)=$ $\sqrt{\left(x_{n+1}-x_{n}\right)^{2}+\left(y_{n+1}-y_{n}\right)^{2}}$, is shown in the inset. We observe immediately that speed remains constant for all individuals due to the linear slope of the data over the entire observation time. The speed given by the mean slope taken from each individual is calculated for all actin concentrations and shown in Fig. 5.5a. Note that we include the whole population here and do not distinguish between motility modes to obtain population wide statistics. We observe that in the absence of actin $(0 \mathrm{mg} / \mathrm{mL})$ average speed is $(9.1 \pm 0.3)$ $\mu \mathrm{m} / \mathrm{s}$. Addition of the actin network to the system results in an immediate drop in mean speed to $\sim 5 \mu \mathrm{m} / \mathrm{s}$ and then decreases only slightly with increasing concentrations.

Mechanical properties of reconstituted actin networks have been studied extensively [73; 74]. In vitro F-actin filaments have a persistence length of $\sim 12 \mu \mathrm{m}$ [52], with a typical length of $20 \mu \mathrm{m}$. F-actin filaments thus form an entangled solution of semi-flexible polymers with average mesh sizes ranging from $\sim 100 \mathrm{~nm}$ to $2 \mu \mathrm{m}$. The mesh size of the actin network, $\xi$ has a quadratic dependence on the actin concentration. Based on previous calculations [75; 76], the mesh size, $\xi$, for randomly arranged fibers, is given by: 

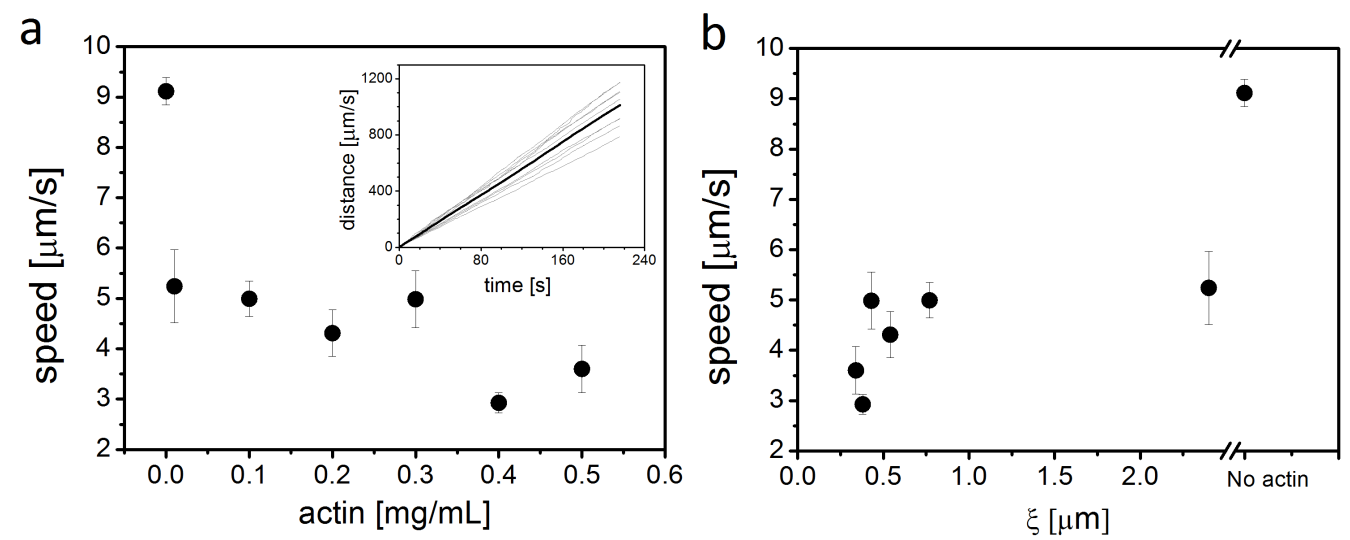

Figure 5.5: a. Average speed of trypanosomes vs. actin concentration. $0 \mathrm{mg} / \mathrm{mL}$ is purely HMI9 complete culture medium (no actin). Inset shows distance vs. time for $0.1 \mathrm{mg} / \mathrm{mL}$ actin environment. b. Average speed vs. mesh size $\xi$ of entangled actin.

\begin{tabular}{|c|c|c|c|c|c|c|}
\hline Actin Concentration $(\mathrm{mg} / \mathrm{mL})$ & 0.01 & 0.1 & 0.2 & 0.3 & 0.4 & 0.5 \\
\hline Mesh size $\xi(\mu \mathrm{m})$ & 2.4 & 0.77 & 0.54 & 0.43 & 0.38 & 0.34 \\
\hline
\end{tabular}

Table 5.1: Mesh size corresponding to actin concentration

$$
\xi=d_{f}\left[\left(\frac{\ln (2)}{c_{a} V_{s}}\right)^{2}-1\right]
$$

Where $d_{f}$ is the diameter of the fibers taken at $8 \mathrm{~nm}$, and the volume fraction is the product of the specific volume of protein $V_{s}$ taken as $0.74 \mathrm{~cm}^{3} / \mathrm{g}$ [69] and the actin concentration $c_{a}$. Actin concentrations and corresponding mesh sizes $\xi$ are given in Table 5.1 .

Plotted against mesh size in Fig. 5.5p, the speed of the trypanosomes seems to increase and reach saturation, it does not however reach the same value as in pure culture medum, despite a mesh size that is about the same as the average cell diameter, suggesting that the cells are still confined at even the lowest concentrations.

Some experimental and theoretical work has shown that flagellated bacteria actually swim faster as the viscosity of the surrounding medium increases due to polymer networks [77; 78; 79]. However this effect is species-specific and depends on the configuration of the cell, in terms of size, flagellar attachment, helical wavelengths etc. For instance, V. alginolyticus, marine bacteria, have two types of flagella: polar (seen emerging at the cell pole), and lateral (additional flagella). V. alginolyticus do swim faster in higher viscosities but only when they express their lateral flagella; swimming exclusively with the polar flagellum results in a steady decrease in swimming speed [80]. This appears to be the case with trypanosomes (Fig. 5.5), who only possess a lateral flagellum.

Remarkably a trypanosome is able make its way through such a dense network, with mean mesh sizes up to eight times smaller than its own body diameter. Indeed previous 


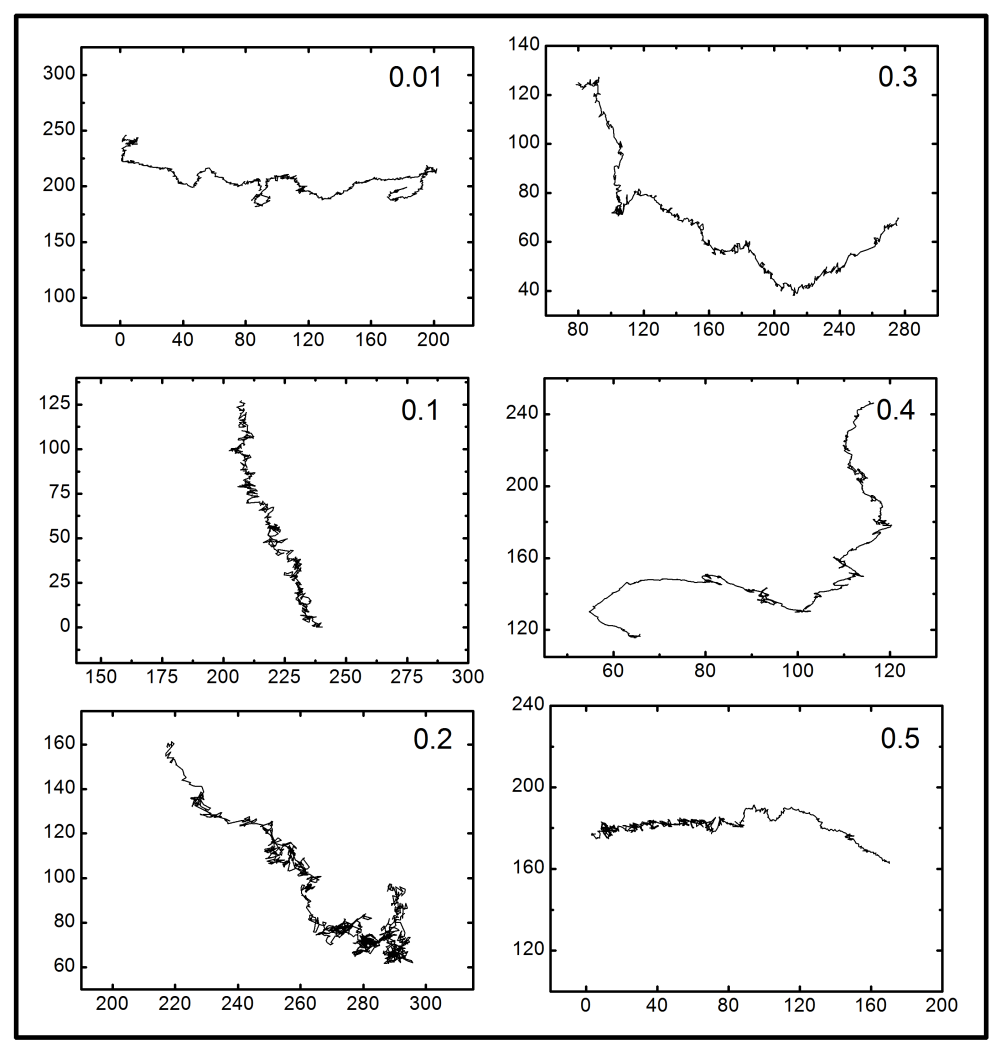

Figure 5.6: Typical trajectories of individual cells at a range of actin concentrations (0.01$0.5 \mathrm{mg} / \mathrm{mL}$ ). Note that at $0.5 \mathrm{mg} / \mathrm{mL}$ the trypanosome appears to have slowed down (from right to left) as indicated by the smaller denser movements in the trajectory. Units are $\mu \mathrm{m}$.

experiments have shown that trypanosomes are able to swim in microfluidic channels that are $1 \mu \mathrm{m}$ in width and $4.5 \mu \mathrm{m}$ in height [81]. Thus the cell may have the ability to tunnel through gaps that are narrower than its own body diameter.

A closer look at the trajectories of the fastest trypanosomes (Fig. 5.6) shows that cell movement does not appear to be significantly altered upon addition of actin to the environment. Without actin, it is clear that the trypanosome has more degrees of freedom, and thus can move faster and in many directions. Overall, increasing concentrations of actin, with narrowing mesh sizes causes cells to be 'trapped' and may enhance persistence of the trajectories by forming a loose tunnel through which the trypanosomes swims (Fig. 5.7). However, the cell body may be undergoing more undulations at smaller time scales in the networks to achieve a net motion. As we will see below, cell locomotion of trapped cells is strongly dependent on the elasticity of the network.

We explore the the degree of persistence further by returning to the analysis from Chapter 4 and examining the motility mode of the trypanosomes with respect to the concentration of actin. The cells are classified as either random walkers (RW), intermediate walkers (IW) or persistent walkers (PW) using the same criteria and find an overall de- 


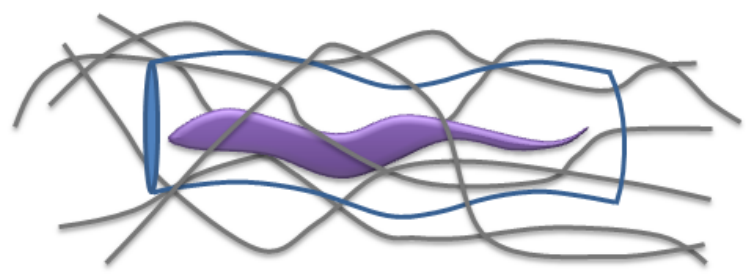

Figure 5.7: Actin network forms a loose pipe around the body through which the cell can move.

crease in the proportion of persistent walkers and a corresponding increase in the number of random walkers upon addition of actin filaments to the environment (Fig. 5.8). Upon addition of actin to the environment, some cells lose persistence by becoming trapped within the network and are therefore classified as random walkers.

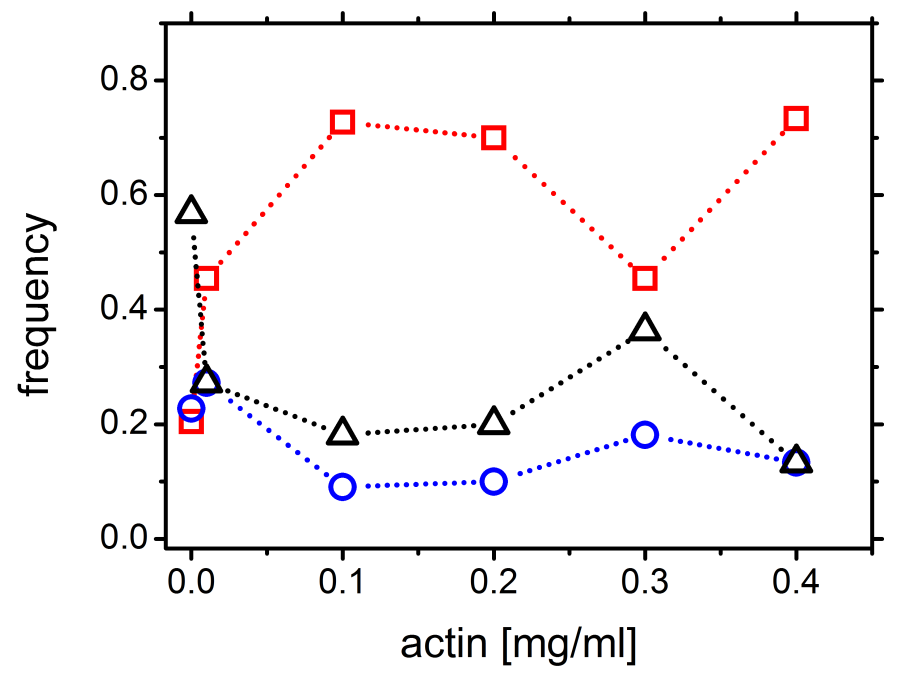

Figure 5.8: Distribution of cells in each motility mode as a function of actin concentration. RW in red, PW in black, IW in blue.

The correlation of cell swimming in these entangled solutions is examined by the mean squared displacement (MSD) versus time shown in Fig. 5.9 for trypanosomes swimming in a range of concentrations. The MSD is given by $\left\langle x(\tau)^{2}\right\rangle=\left\langle(r(t)-r(t-\tau))^{2}\right\rangle$, where $\left\langle x^{2}\right\rangle$ is the MSD, $r$ is position, and $\tau$ is the 'profile' time.

The mean scaling exponent, $\alpha$, (where $\left.\left\langle x(\tau)^{2}\right\rangle=4 D t^{\alpha}\right)$ is shown in Fig. 5.10 as a function of concentration, and as a function of mesh size in the inset. We observed a small increase in the average scaling exponent with increasing mesh size after which scaling is recovered nearly to the 'no-network' scaling (i.e at $0 \mathrm{mg} / \mathrm{mL}$ ).

In the MSD curves of Fig. 5.9, the average RW and PW curves are also shown in red and black respectively. At the small time scales, all cells have about the same scaling, while at longer time scales, the scaling exponent diverges for the motility modes. In- 

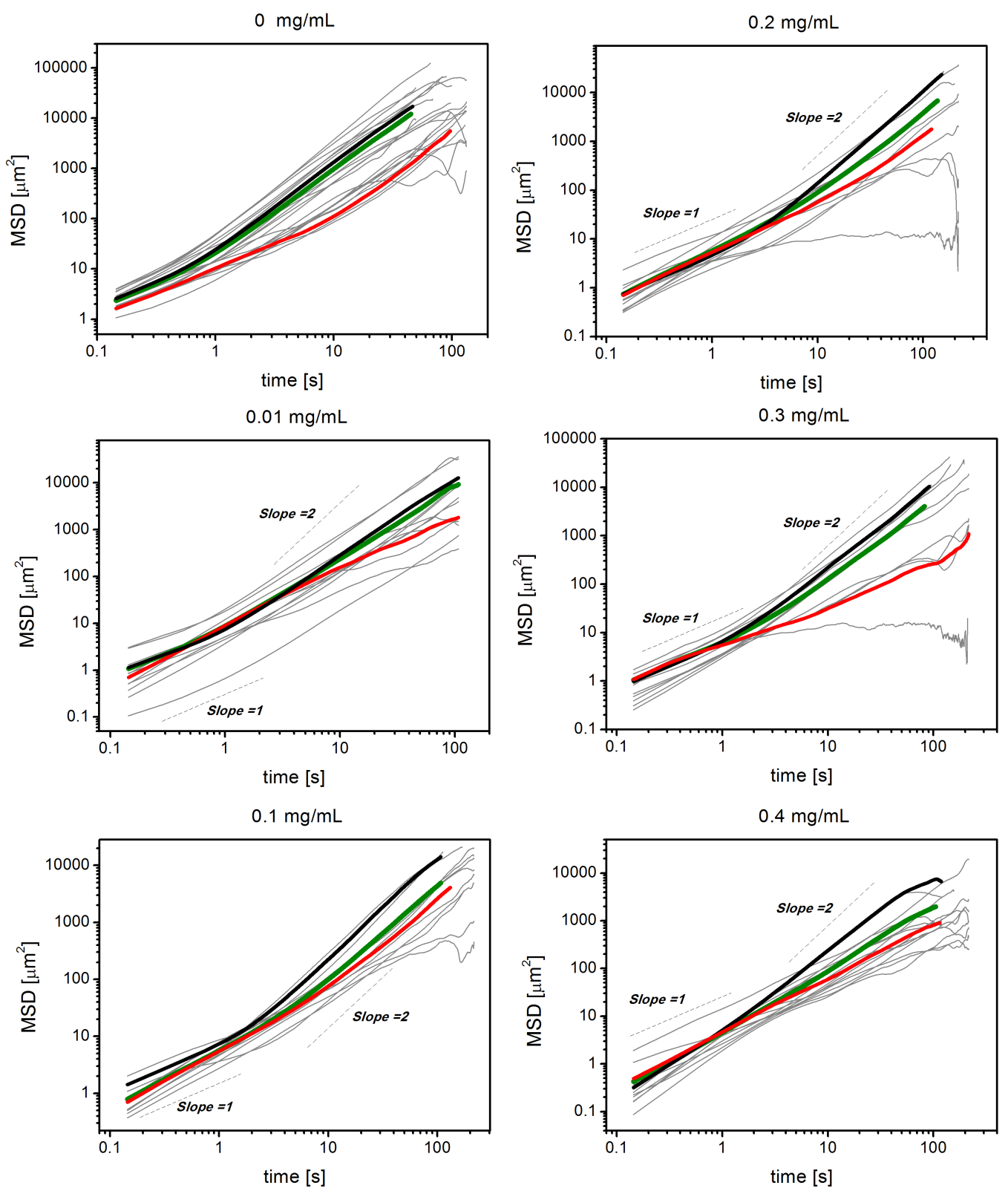

Figure 5.9: Mean squared displacement vs. time for trypanosomes swimming through networks of increasing actin concentration. Mean MSD shown in green. Random walker and persistent walker averages shown in red and black respectively. Dashed lines show slopes, note that a crossover in slopes is seen mainly for the persistent walkers.

terestingly, as actin concentration is increased, while persistent cells maintain the same correlation, random walkers show a gradual reduction in slope. We comment further on the scaling below. 


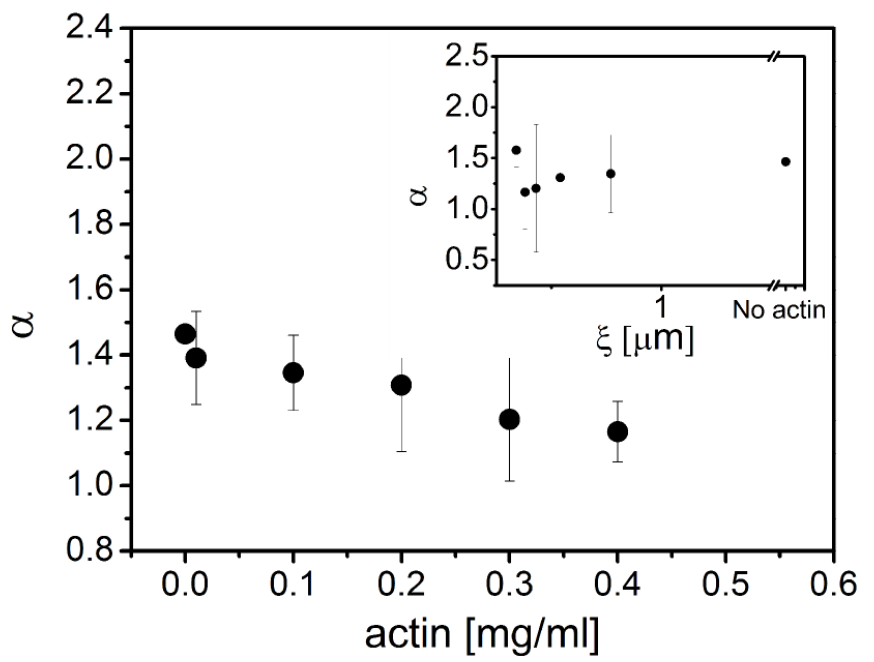

Figure 5.10: Scaling exponent as a function of actin concentration and mesh size (inset).

\subsubsection{Collagen}

Fig. 5.11 shows that collagen fibers do not form homogeneous networks; some areas appear to be much more sparsely populated with collagen fibers than others.

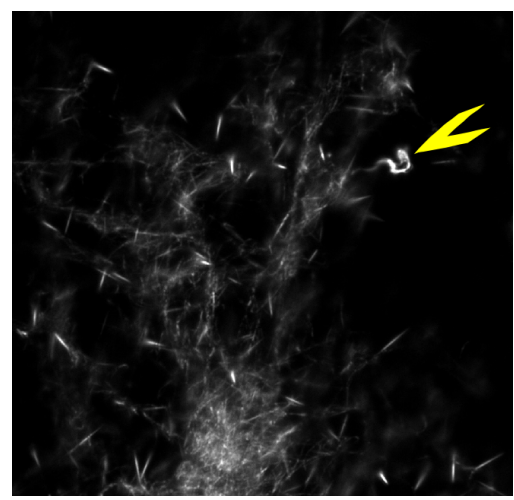

Figure 5.11: Darkfield microscopy image taken of a trypanosome swimming in a collagen network of $0.1 \mathrm{mg} / \mathrm{ml}$. Inhomogeneities in the network are visible. Yellow arrow points to a trypanosome

Addition of collagen to the environment also results in an immediate drop in cell speed. However, overall network inhomogeneities are reflected in Fig. 5.12, which does not show an apparent decrease after the addition of collagen. In addition the spread of the data (reflected in the error bars) is larger than that of the actin data; indicative of network inhomogeneities.

From Eq. 5.1, assuming an average fiber diameter of $200 \mathrm{~nm}$ [69], the mesh sizes for the collagen concentrations that were studied are given in Table 5.2 .

The collagen network mesh sizes are up to 40 fold larger than the actin mesh sizes 
a

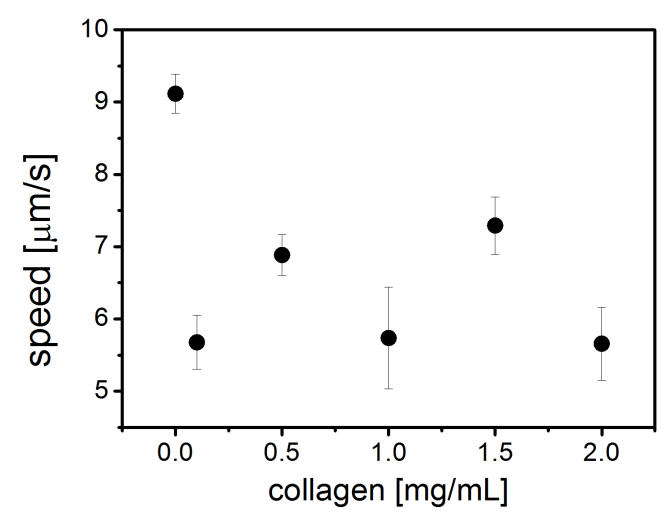

b

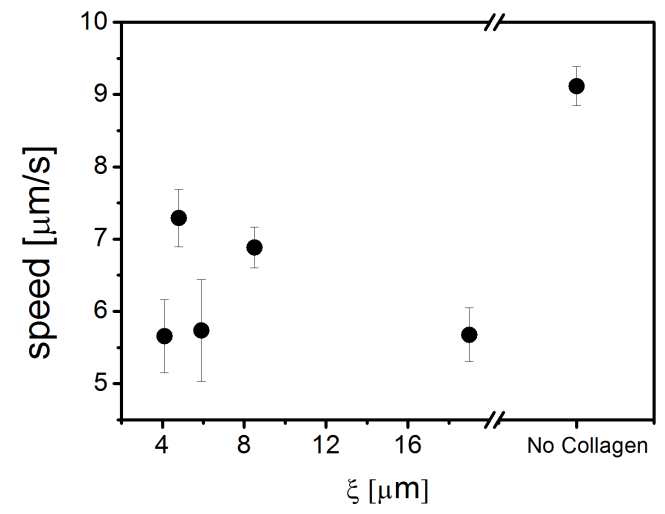

Figure 5.12: Average speed of trypansomes vs. collagen concentration and mesh size. 0 $\mathrm{mg} / \mathrm{ml}$ is purely HMI9 complete culture medium.

\begin{tabular}{|c|c|c|c|c|c|}
\hline Collagen Concentration $(\mathrm{mg} / \mathrm{mL})$ & 0.1 & 0.5 & 1.0 & 1.5 & 2.0 \\
\hline Mesh size $\xi(\mu \mathrm{m})$ & 19 & 8.5 & 5.9 & 4.8 & 4.1 \\
\hline
\end{tabular}

Table 5.2: Mesh size corresponding to collagen concentration

explored and this is reflected in the slightly greater mean cell speed in collagen than in actin $(\sim 6.5 \mu \mathrm{m} / \mathrm{s}$ vs. $\sim 5 \mu \mathrm{m} / \mathrm{s})$. The cells are again sorted into their respective motility modes and their distributions are shown as a function of collagen concentration in Fig. 5.13. We see that upon addition of collagen, the number of PWs drops dramatically (and sometimes to zero).

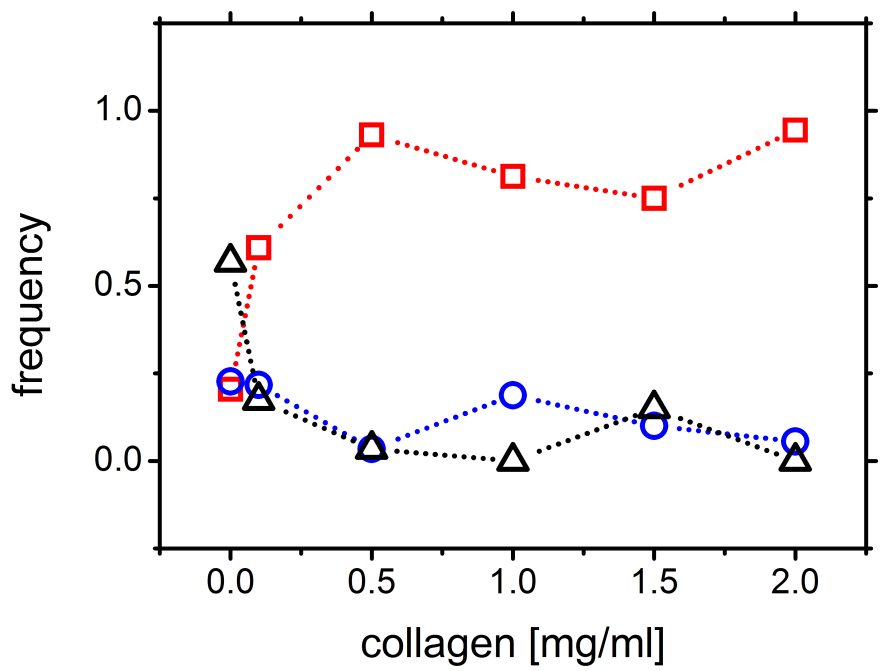

Figure 5.13: Distribution of cells in each motility mode as a function of collagen concentration. RW in red, PW in black, IW in blue.

MSDs are shown in Fig. 5.14 for trypanosomes swimming in collagen networks of 
increasing collagen concentrations. The average MSD, PW average and RW average are highlighted in thicker green, black and red lines. Overall, we find that as the concentration is increased, the mean MSD converges with the slope of the random walkers.
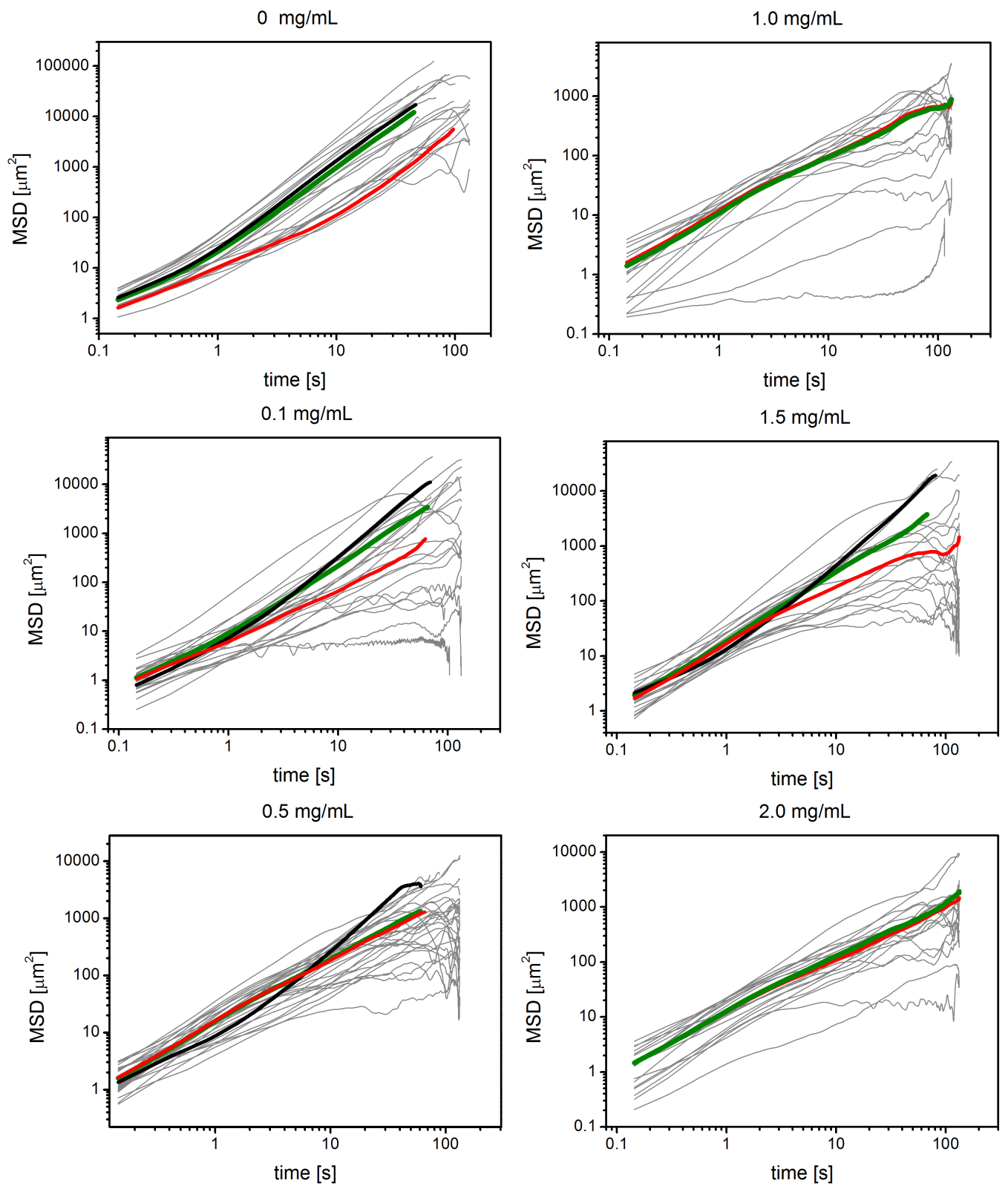

Figure 5.14: Mean squared displacement vs. time for trypanosomes swimming through networks of increasing collagen concentration. Mean MSD shown in green. Random walker and persistent walker averages shown in red and black respectively. Note that a crossover in slopes is seen mainly for the persistent walkers.

The scaling exponent $\alpha$, as defined above derived from the MSD, is shown in Fig.5.15 as a function of collagen concentration and mesh size (inset). One observes a steady drop in the scaling as the concentration is increased - in sharp contrast to the small linear de- 
cay observed in the actin networks (Fig. 5.10). Indeed, we see that soon after addition of collagen to the environment, at collagen concentrations above $0.5 \mathrm{mg} / \mathrm{mL}$ the average scaling exponent falls below one, indicative of subdiffusive behaviour.

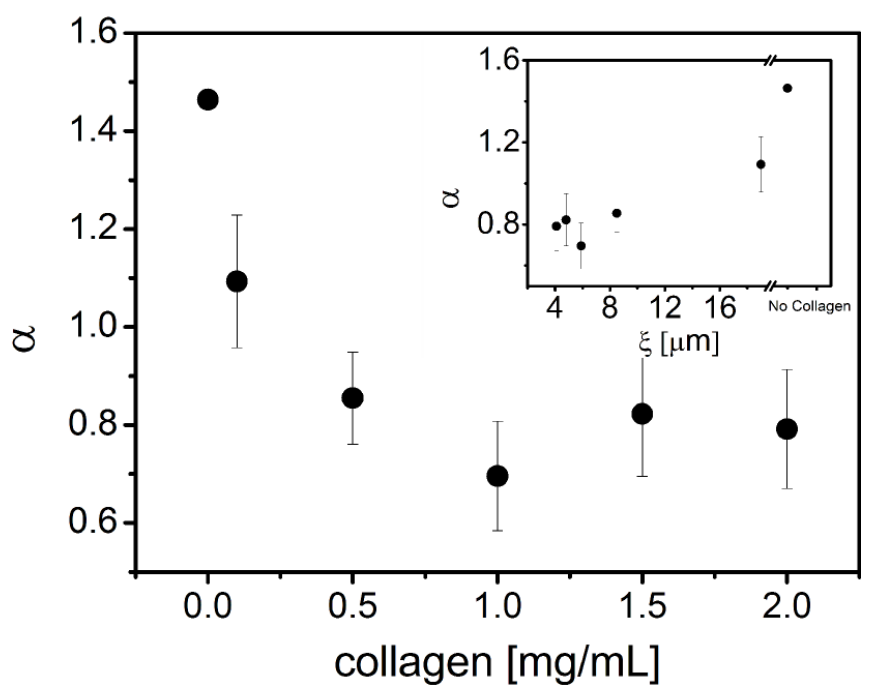

Figure 5.15: Mean scaling exponent $\alpha$ as a function of collagen concentrations and corresponding mesh sizes (inset).

\subsubsection{Dextran}

We look now at the effect of viscosity on cell motility. While viscosity of gels resulting from physical networks is difficult to measure and has resulted in varied reports through rheological studies, it can be well controlled and measured with the addition of 'thickening' agents such as methylcellulose and dextran.

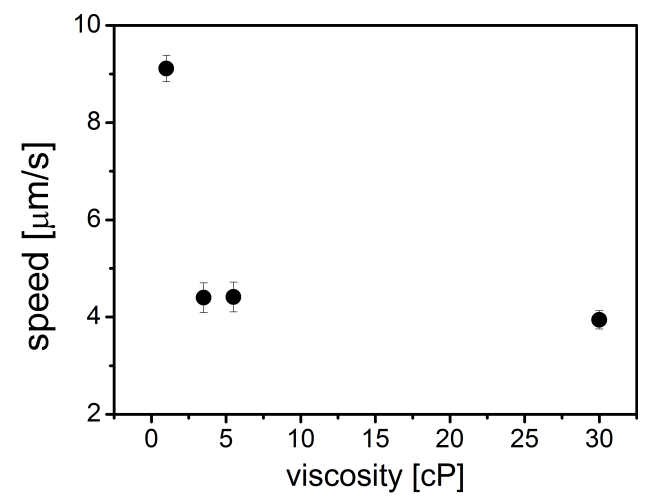

Figure 5.16: Average speed of trypanosomes vs. viscosity (controlled by dextran concentration, corresponding to 0, 5, 8, and 25\% w/v Dextran in HMI9, see Experiment section). The viscosity of $1 \mathrm{cP}$ corresponds to HMI9 complete culture medium without Dextran.

As in Fig. 5.5 and Fig. 5.12 we see that the speed of the cells decreases in the presence 
of an external viscosity tuning agent such as dextran, shown in Fig.5.16. Previous work has shown that trypanosomes do exhibit higher speeds when the viscosity is increased in gels of methylcellulose (without physical networks) [82] and that trypanosomes reach a maximum speed at blood-matched viscosities [30]. While overall speed may be reduced, trypanosome mobility is preserved in viscosities shown to immobilize other microorganisms [30; 77], as shown in Fig. 5.17. Indeed even at a viscosity of $30 \mathrm{cP}$ we observe some directional motion.
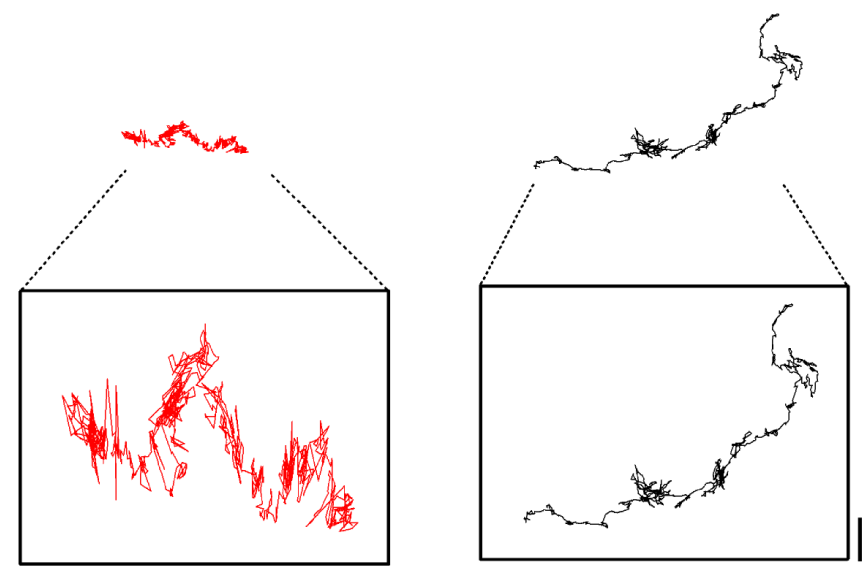

Figure 5.17: Trypanosome trajectories in $8 \% \mathrm{w} / \mathrm{v}$ (black) and $25 \% \mathrm{w} / \mathrm{v}$ (red) dextran plotted at the same scale, scale bar is $25 \mu \mathrm{m}$. Insets: trajectories are zoomed in.

As observed in actin and collagen networks, the number of persistent walkers is reduced dramatically upon addition of dextran to the suspending solution (Fig. 5.18).

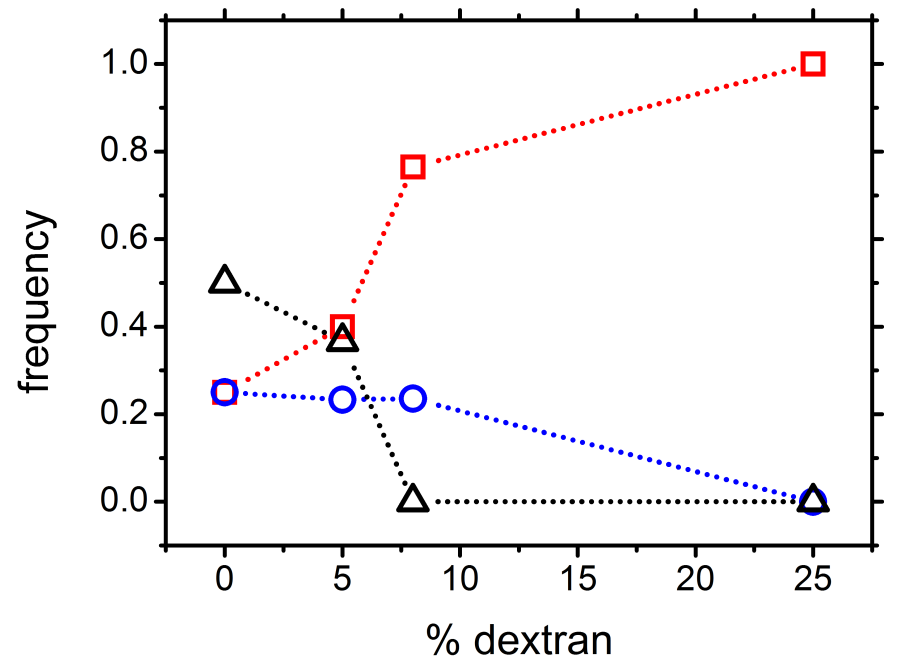

Figure 5.18: Distribution of cells in each motility mode as a function of $\%$ dextran. RW in red, PW in black, IW in blue. 

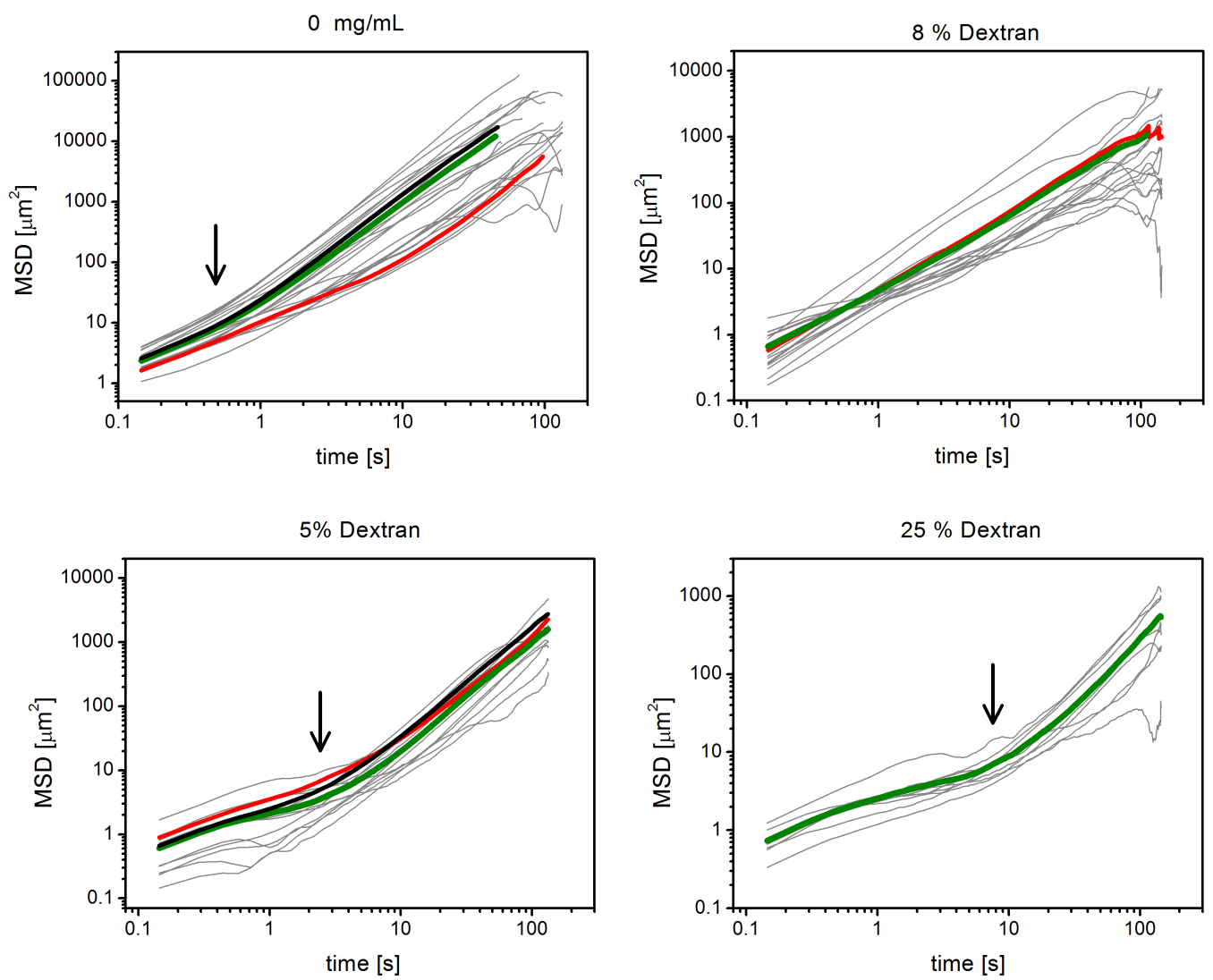

Figure 5.19: Mean squared displacement (MSD) vs. time for trypanosomes swimming through a range of viscosities tuned by varying dextran concentrations. Mean MSD shown in green. Random walker and persistent walker averages shown in red and black respectively. Note that a crossover in slopes is seen (indicated by black arrows).

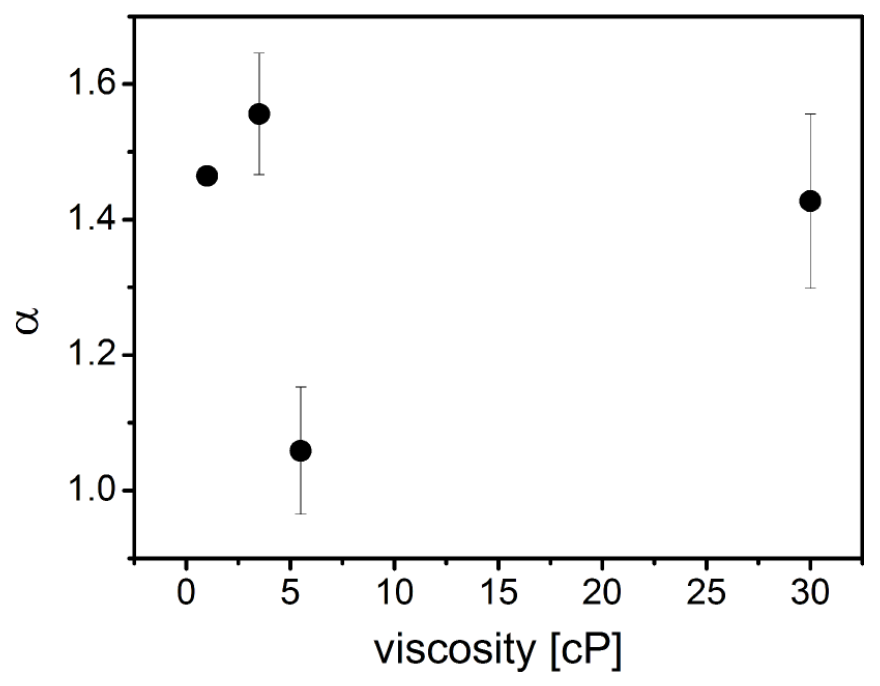

Figure 5.20: Mean scaling exponent $\alpha$ as a function of dextran concentrations. 
Fig. 5.19 shows MSD curves for populations of trypanosomes in a range of dextran concentrations. While we observe an overall decrease in the scaling with increasing dextran (Fig. 5.20), the MSDs (Fig. 5.19) reveal a strong crossover in slope, not seen in the networks and also shows that the crossover is shifted as the viscosity is increased.

\subsubsection{Comparison of environments}

Specific time scales conferred to trypanosome motility due to the environment is investigated in Fig. 5.21. The slope of the MSDs are shown and therefore illustrate how the scaling exponent $\alpha$ evolves over time.
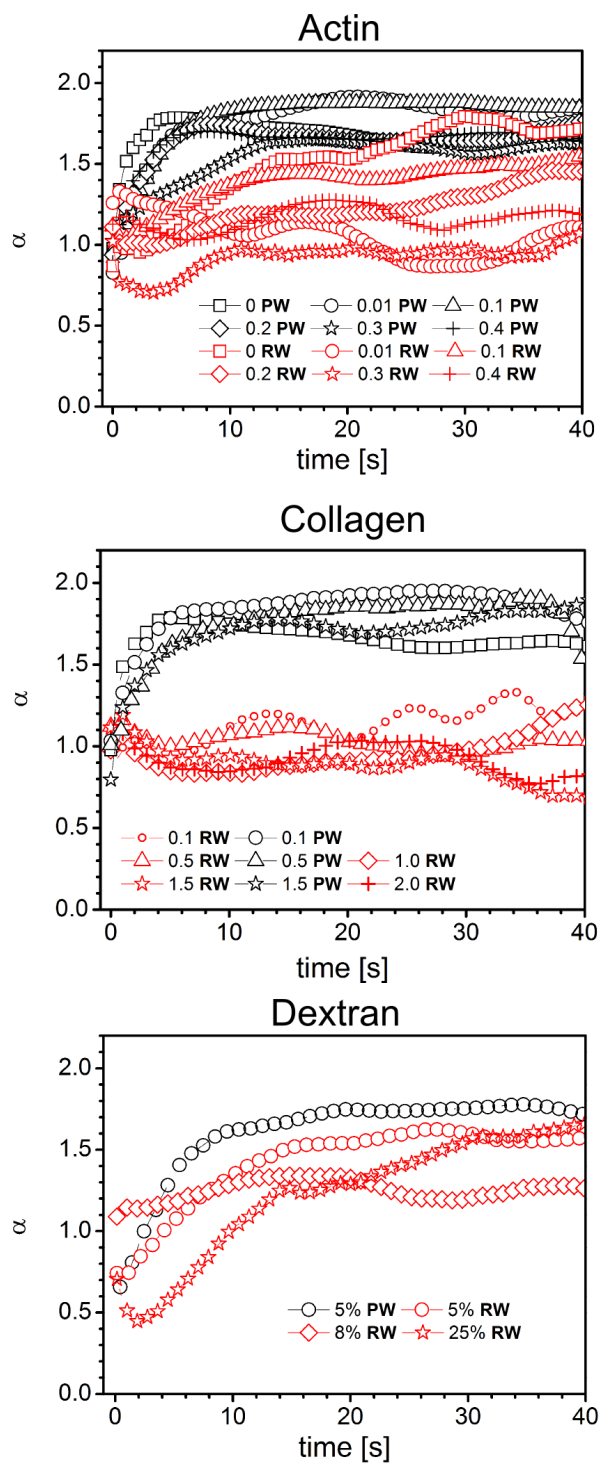

Figure 5.21: Time evolution of scaling of average PW (black)and RW (red) curves (20point smoothing of derivative of MSD). 
In actin, we observe that at small times scaling is diffusive to subdiffusive with $0.7 \leq$ $\alpha \leq 1$ - due to the fast body undulations described in Chapter 4. Scaling rapidly increases for the PWs across in all concentrations. Contrastingly, RWs maintain a constant slope, but this overall slope is reduced as the concentration of actin is increased.

In collagen networks, we see again that at small time scales where $t \leq 4 \mathrm{~s}, \alpha$ is close to unity and then makes a jump and remains at the same level in subsequent time intervals. In collagen, PWs and RWs maintain a much more distinct scaling as compared to the cells in actin. We do not observe the same gradual reduction in RW slopes as seen in actin. This may in part be explained by the larger range of mesh sizes used in the collagen network. However, the mean scaling exponent at a collagen concentration of $2.0 \mathrm{mg} / \mathrm{mL}(\xi=4.1$ $\mu \mathrm{m})$ is 1 - significantly lower than a comparably spaced actin network of $0.01 \mathrm{mg} / \mathrm{mL}(\xi=$ $3 \mu \mathrm{m})$. Therefore collagen fibers seem to have a direct and immediate effect on directional persistence compared to actin - reflecting the elasticity of the surrounding networks.

The mean slope of the MSDs shows dramatically different behaviour in dextran than that observed in networks. It begins with anticorrelated motion at the very short time scale and then reaches a slope of about 1.4 and is more or less maintained. At $8 \% \mathrm{w} / \mathrm{v}$ dextran, we see instead a fairly constant slope maintained just above 1, indicating that the cell motion falls into a diffusive regime. As the viscosity increases, the slope is low again and crosses over to correlated motion only at longer times. This indicates that while the cells undergo highly uncorrelated motion at the smaller time scales, an overall direction is maintained. As shown in Fig. 5.21, in Dextran, RWs exhibit similar scaling to PWs at longer times. The crossover in slopes point to inherent time scales conferred to cell motility by the environment.
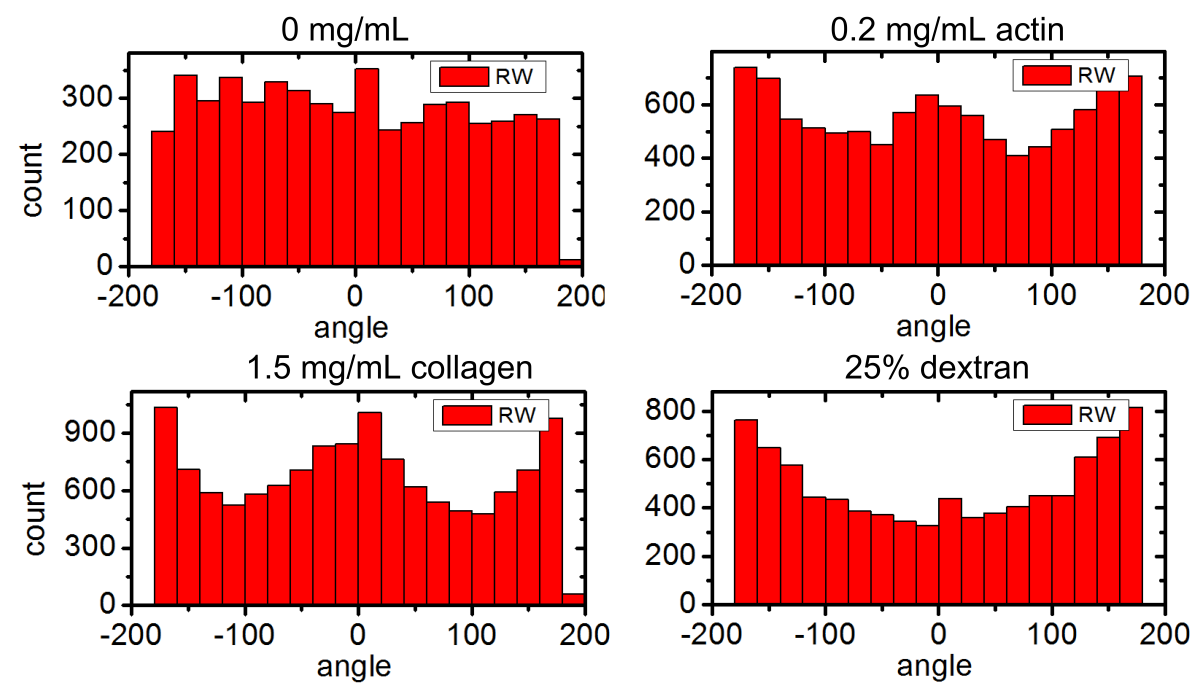

Figure 5.22: Population turn angle distributions for random walkers in culture medium, actin, collagen and dextran at specified concentrations. 
The inclusion of networks and thickening agents resulted in an increase in the proportion of RWs, we therefore look more closely at their turn angle distributions in Fig. 5.22 In HMI9 medium $(0 \mathrm{mg} / \mathrm{mL})$, a uniform turn angle distribution is seen, while in the presence of networks $(0.2 \mathrm{mg} / \mathrm{mL}$ actin, $1.5 \mathrm{mg} / \mathrm{mL}$ collagen) show more pronounced peaks near $0,-180$ and 180 . These angles show that the cell center of mass is moving forward or from side to side, and such movement is enhanced with increasing confinement as shown schematically in Fig. 5.23. This explains the somewhat anticorrelated behaviour represented by scaling exponent $\alpha \leq 1$ for RWs.
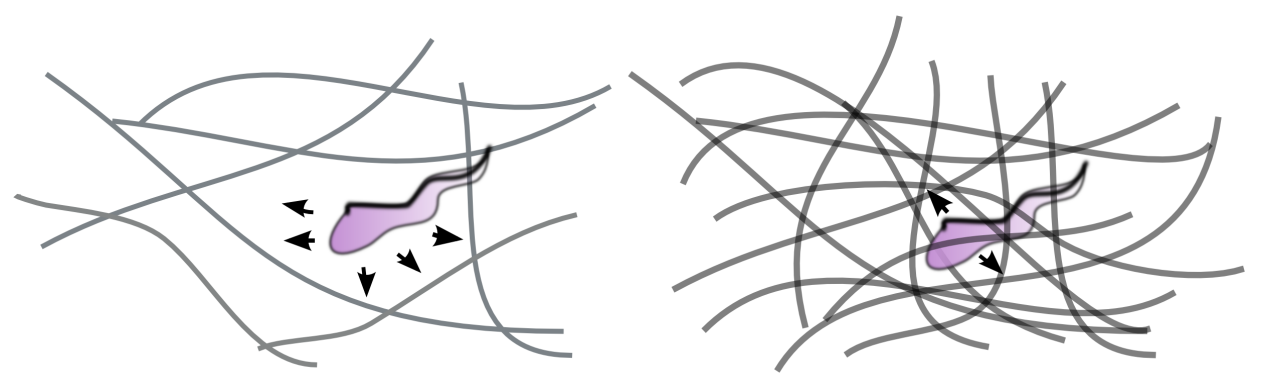

Figure 5.23: Trypanosomes in denser networks become more trapped and show a tendency to 'rock' back and forth. While there is no physical network in the case of dextran, the increased viscosity has a similar impact on cell motion.

With $25 \% \mathrm{w} / \mathrm{v}$ dextran, the highest viscosity, persistence is lost and we observe higher peaks only near -180 and 180 in random walkers. The peak at the center of the distribution has nearly disappeared - indicating that a back and forth rocking of the cell dominates over any forward movement.

\subsubsection{Trypanosomes as active probes for network properties}

The experiments presented in this chapter so far provide insight into the swimming behaviour of trypanosomes in environments of tunable viscosity and mesh size. We found firstly that while the addition of any viscosity changing agent resulted in an immediate drop in mean speed, further addition resulting in smaller decreases in speed.

Passive diffusive fluctuations of microsphere probes of radius $a$ are used in microrheology to extract the elastic modulus, $G^{\prime}$, of a polymer network [83; 84]:

$$
G^{\prime} \approx \frac{2 k_{b} T}{a\left\langle\Delta r^{2}\right\rangle}
$$

where $k_{b}$ is Boltzmann's constant, $T$ is the temperature and $a$ is the diameter of the probe. Tracking the probe motion due to thermal fluctuations requires probes of the same dimensions as the mesh size of the network, and fluctuation detection algorithms [85]. However, probes that travel through a network (when its dimensions are small enough to slip through the entangled filaments) can be used to estimated the mesh size 
of a network using the square root of the MSD, $\sqrt{\left\langle\Delta r^{2}\right\rangle}$ [83].

In addition to characterizing the behaviour of trypanosomes in tunable environments, results presented into this chapter suggest that trypanosomes could be used as probes to study the physical properties of the environments themselves. We find however that taking $\sqrt{\left\langle\Delta r^{2}\right\rangle}$ for trypanosome movements generally yields values from 2 to 30 fold greater than the geometrical mesh size calculated from Eq. 5.1 indicating that the cell is able to make its way through networks with gaps smaller than its own body size.

This discrepancy arises because two additional factors come into play in the case of active probes (such as self propelled microorganisms). First, the motion is not purely thermal, and second the force exerted by the active probe may deform network fibers.

We calculate instead the radius of gyration, $R_{g}$, which gives us a measure of how far a trypanosome can travel. Often used in polymer physics, $R_{g}$ is commonly used to measure the radius of the sphere that a polymer would occupy. $R_{g}$ is given by [42]:

$$
R_{g}=\sqrt{\frac{1}{n(t)} \sum_{i=1}^{n}\left(r_{i}-r_{c m}\right)^{2}}
$$

with $r_{c m}=\frac{1}{n(t)} \sum_{i=1}^{n} r_{i}$ is the center of mass trajectory and $r_{i}$ represents the $i=1, \ldots, n(t)$ position for an individual.

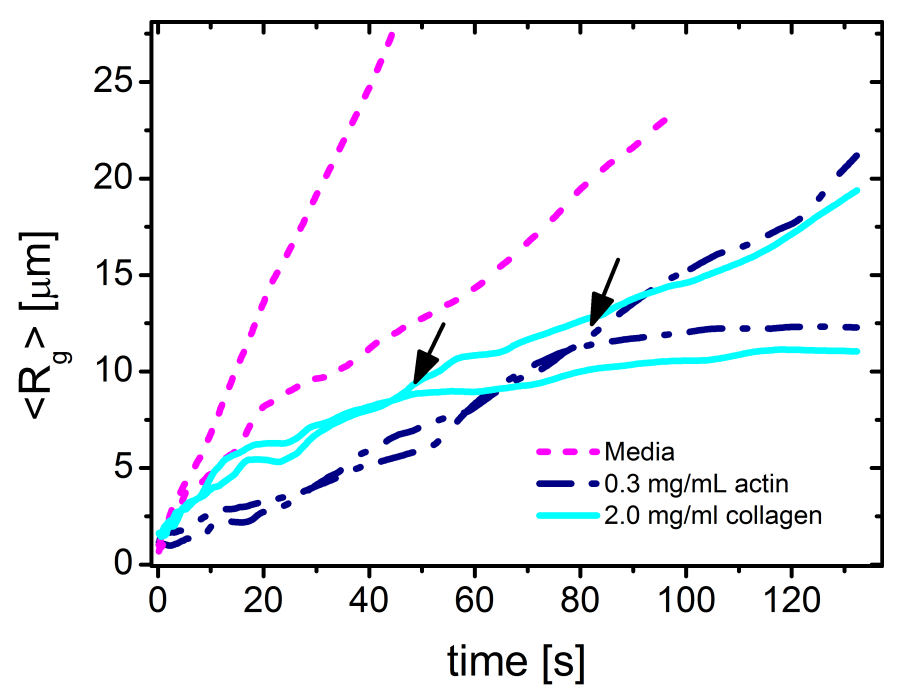

Figure 5.24: Typical $R_{g}$ curves for trypanosomes in collagen, actin and medum, as described in the text.

If, within the observation interval, the movement of the trypanosome is on average restricted to a certain radius, we observe that the radius of gyration saturates following an initial rise. Conversely if a cell after some initial movement finds a tunnel, it may drill its way through the network persistently. This description is illustrated in Fig. 5.24. In collagen and actin, we see that all $R_{g}$ curves begin with the same slope, but cells that are trapped display a saturation in $R_{g}$, while it is monotonically increasing for tunneling 
cells. In culture medium, a comparison of PWs and RWs shows that the slopes diverge at a much shorter time interval.

For each concentration the average $R_{g}$ curves for such 'restricted' trypanosomes (i.e saturated) are given in Fig. 5.25 $\mathrm{a}$ and Fig. 5.25 $\mathrm{p}$ in actin and collagen respectively. $R_{g}$ curves for tunneling trypanosomes in actin and collagen are shown in Fig. $5.25 \mathrm{k}$ and Fig. 5.25d respectively.
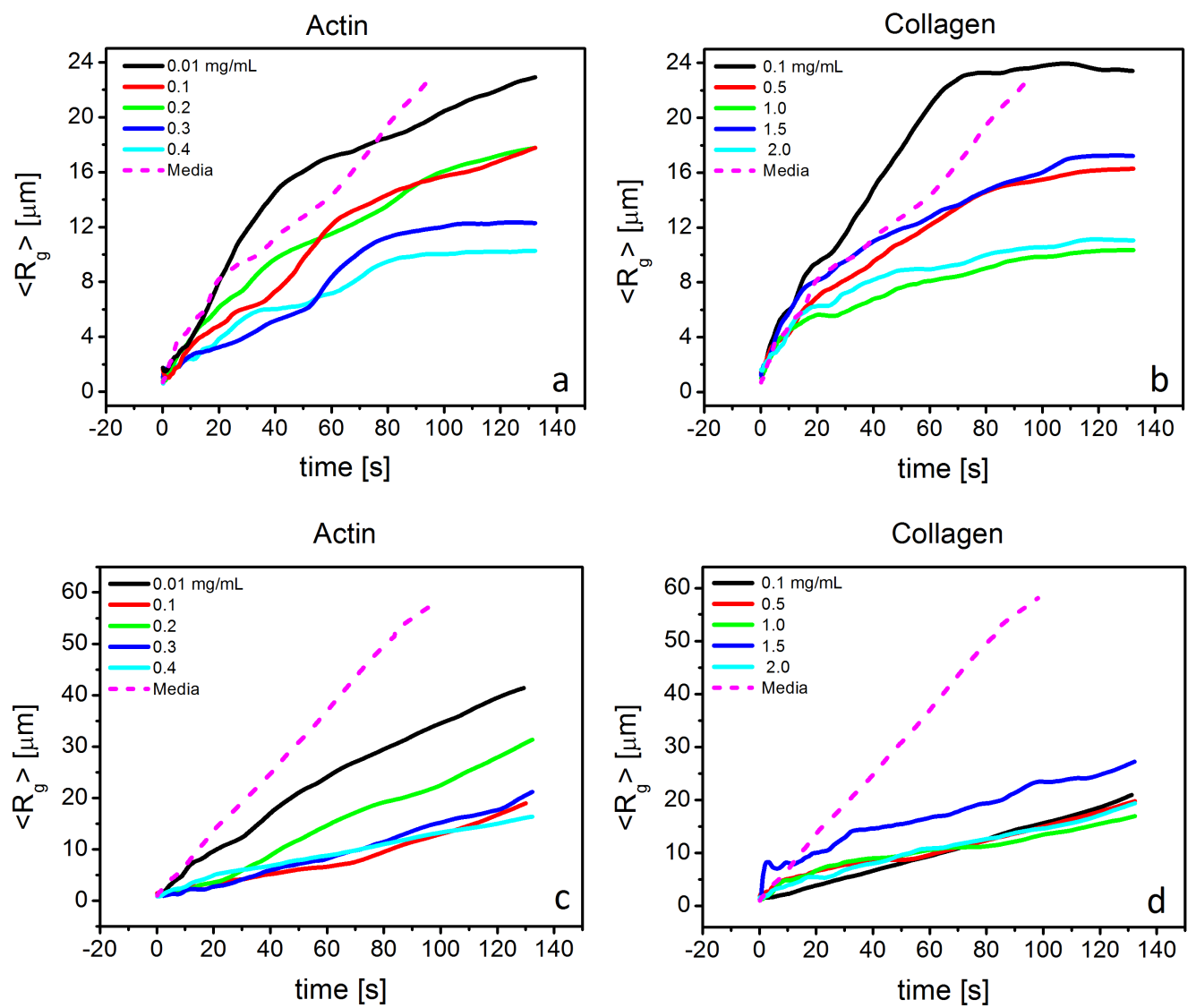

Figure 5.25: a \& b. Mean $R_{g}$ for trajectories that appear to saturate in actin and collagen at a range of concentrations. c \& d. Mean $R_{g}$ for cells that are persistent and thus 'tunnel' through the networks. Data for cell swimming in medum are also shown.

The saturation level of the radius of gyration, $R_{g_{s a t}}$ in Fig. 5.25 represents a physical limit that is imposed on cell movement and may be a measure of the mesh size of the network. Indeed we find that for collagen $\left\langle R_{g_{s a t}}\right\rangle / \xi \approx 2$ and for actin $\left\langle R_{g_{s a t}}\right\rangle / \xi \approx 24$. These ratios indicate that from $R_{g_{s a t}}$ we may estimate the mesh size of the collagen network. In the case of actin, the ratio indicates that the cell is not limited to the mesh formed by the entangled solution suggesting that network is less stiff than collagen networks.

Since $R_{g}$ yields an estimate of the area which the trypanosome cover while being confined within the network, we draw an analogy to a minimal bead spring model and additionally extract information about the mechanical properties of the network. The 


\begin{tabular}{|c|c|c|c|}
\hline $\begin{array}{c}\text { Actin concentration } \\
(\mathrm{mg} / \mathrm{mL})\end{array}$ & $\kappa(\mathrm{N} / \mathrm{m})$ & $\begin{array}{c}\text { Collagen } \\
\text { concentration } \\
(\mathrm{mg} / \mathrm{mL})\end{array}$ & $\kappa(\mathrm{N} / \mathrm{m})$ \\
\hline 0.01 & $3.1 \times 10^{-7}$ & 0.1 & $3.1 \times 10^{-7}$ \\
\hline 0.1 & $3.9 \times 10^{-7}$ & 0.5 & $4.3 \times 10^{-7}$ \\
\hline 0.2 & $4.0 \times 10^{-7}$ & 1.0 & $6.0 \times 10^{-7}$ \\
\hline 0.3 & $5.7 \times 10^{-7}$ & 1.5 & $4.1 \times 10^{-7}$ \\
\hline 0.4 & $6.9 \times 10^{-7}$ & 2.0 & $6.3 \times 10^{-7}$ \\
\hline
\end{tabular}

Table 5.3: Estimated spring constant $\kappa$ for different networks.

constant $\kappa$ characterizes the network stiffness with applied force $F=\kappa x . F$ is estimated as the force generated by the trypanosome, previously measured to be $\sim 5 \mathrm{pN}$ [81]. Using $\left\langle R_{g_{s a t}}\right\rangle$ as a 2D estimate for the distance the trypanosome travels through the network, we approximate the 1D displacement to $\sqrt{2}\left\langle R_{g_{\text {sat }}}\right\rangle$.

Table 5.2.5 shows the values obtained from this minimal model to estimate the spring constant (or stiffness) of the actin and collagen networks studied.

The elasticity of the network may be estimated as the ratio $\kappa / a$ where $a$ is the radius of the active trypanosome $(\sim 3 \mu \mathrm{m})$ probe. The value of $\kappa$ from Table 5.2.5yields a value of $\sim 0.13$ and $\sim 0.21 \mathrm{~N} / \mathrm{m}^{2}(\mathrm{~Pa})$ for networks of $0.2 \mathrm{mg} / \mathrm{mL}$ actin and $2.0 \mathrm{mg} / \mathrm{mL}$ collagen respectively. Previous microrheological experiments have shown that a concentration of $0.25 \mathrm{mg} / \mathrm{mL}$ actin corresponds to an elastic modulus of $2 \mathrm{~Pa}$ [73], and a collagen gel of $2.3 \mathrm{mg} / \mathrm{mL} 3 \mathrm{~Pa}$ [86]. The relative ratio between the actin and collagen networks is excellent as compared to previous reports. More specific force measurements exclusively for cells that exhibit a saturating radius of gyration may further improve these results. The method of 'active microrheology' discussed here using self propelled microorganisms is therefore in reasonable agreement with previously established results. Such a measurement is a rapid and efficient method to estimate the elasticity of the environment in which a microorganism is swimming.

\subsection{Summary}

The effects of environments of tunable mesh size and viscosity on trypanosomes motility were studied in this chapter. In general we observed a decrease in the overall speed of trypanosomes upon addition of these tuning agents.

We suggest that the movement of active microorganisms such as trypanosomes can be used a measure of collagen mesh size. Further, the concept of active microrheology, using self propelled probes (trypanosomes), was introduced to estimate the elastic modulus of surrounding polymer networks by distinguishing cells exhibiting different modes of motility.

Finally, the addition of dextran to the culture medium showed that increasing viscosity resulted in a back and forth rocking of cell movement resulting in a decrease in 
correlation at the shorter time scale while still maintaining a degree of directionality on the longer time scale. Notably, trypanosomes are able to swim through high viscosities previously shown to immobilize other microorganisms [77]. These studies may shed light on further aspects on the complex motility mechanisms of trypanosomes in viscoelastic environments. 


\section{Motility in flow}

In the mammalian host, trypanosomes proliferate within the bloodstream. Blood flow within capillaries is in the order of $\mathrm{mm} / \mathrm{s}$, and within the aorta up to $\mathrm{m} / \mathrm{s}$. Such high flow velocities are orders of magnitude higher than the propulsive speed of trypanosomes. A survey of blood stream form trypanosome motility thus requires us to examine trypanosome motility in flow. These studies are interesting both from a medical perspective and from a biophysical perspective to further our understanding of low Reynolds number swimmers in the presence of pressure driven flow. Both theoretical and experimental studies, have dealt with hydrodynamic interactions with boundaries [87] and between microorganisms leading to cooperative behaviour [ 88 ; 89], however little has been done to examine the effects of flow in detail. Upstream swimming of E. coli has been demonstrated [90] in microfluidic channels. Other physical studies with external flow fields on microorganisms have been limited to studying shear effects on adhesive cells.

In this chapter we discuss the motion of single trypanosomes suspended in culture medium in microfluidic channels with controlled flow conditions. We distinguish the role of self-propulsion from purely hydrodynamic effects by drawing a comparison between live and immobilized trypanosomes.

As described in Chapter 2 for the length scales of interest in this thesis, inertial forces are negligible and viscous forces dominate fluid dynamics. Thus, at low Reynolds numbers, flow is predictable and laminar in contrast to turbulent flow. Neighboring streamlines flow parallel to each other in the case of laminar flow, whereas streams are mixed chaotically in turbulent flow.

In flow, the influence of boundary conditions is significant affecting in particular the relation between pressure gradients and volumetric flow rates. The no-slip boundary condition (velocity, $U=0$, at channel walls), yields a parabolic Poiseuille flow profile with the fluid in the center of the channel having the largest velocity:

$$
U=U_{\max }\left[1-\frac{r^{2}}{\left(r_{c} / 2\right)^{2}}\right]
$$

where $U_{\max }$ is the maximum velocity reached, $r$ is the distance from the center and $r_{c}$ is the diameter of the pipe. Fig. 6.1 illustrates a finite element simulation (FEM) performed in COMSOL showing the velocity field in a cross section of a symmetric channel 
for some of the $U_{\max }$ values studied below.
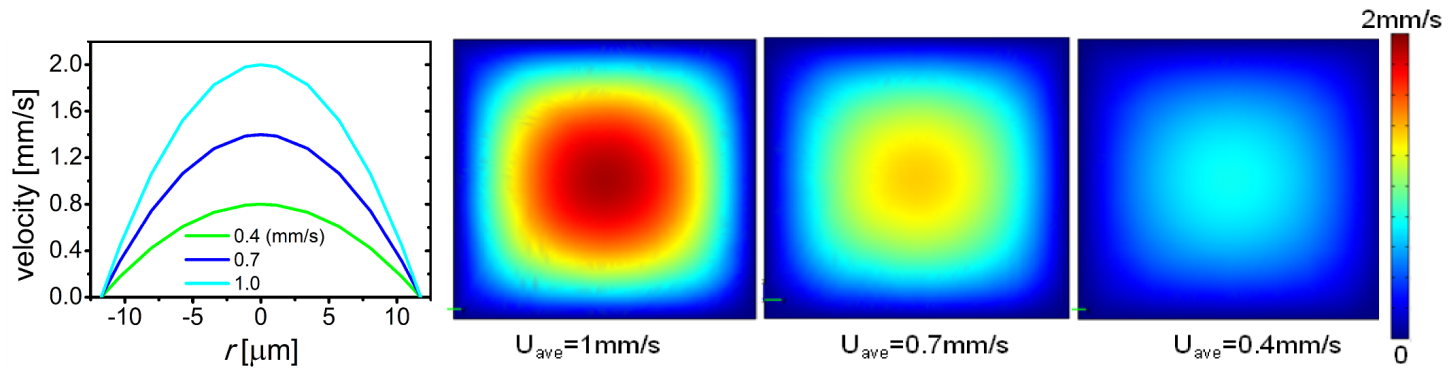

Figure 6.1: Left: flow velocity profile, Right: flow field in a cross section of a symmetric channel from FEM simulation.

The boundary condition also results in maximum shear rate $\dot{\gamma}$ at the walls, which decreases linearly toward the center of the channel:

$$
\dot{\gamma}(r)=\left[\frac{2 U_{\max }}{\left(r_{c} / 2\right)^{2}}\right]|r|
$$

We begin by outlining the experimental setup and analysis methods and then go on to present our findings on trypanosome motility in flow.

\subsection{Experiment}

\subsubsection{Microfluidics}

Microfluidics has emerged as an extremely useful scientific platform to mimic biological environments in order to study microorganisms. It allows for precise control of flow velocities in small volumes ( $\mu \mathrm{L}$ to $\mathrm{pL}$ ) thus presenting an ideal tool for a study of microorganisms in a flow field.

Microfluidic channels were prepared as previously reported [91] using photolithography followed by soft lithography (illustrated below in Fig. 6.2). Briefly:

1. Silicon wafers were coated with photoresist (SU8-50, Micro Resist Technology GmbH, Germany) using a spin coater with the following protocol: ramp in $10 \mathrm{~s}$ to $700 \mathrm{rpm}$, ramp in $30 \mathrm{~s}$ to to $450 \mathrm{rpm}$ and hold for $30 \mathrm{~s}$. This was followed by a soft-bake step at $65^{\circ} \mathrm{C}$ for 10 minutes and then $95^{\circ} \mathrm{C}$ for 45 minutes resulting in a film thickness of $\sim 23 \mu \mathrm{m}$.

2. The wafer was then covered with a photo mask (designed on AutoCAD, AutoDesk Inc., and printed by JD Photo-Tools, UK) and illuminated with UV light ( $\lambda=365 \mathrm{~nm}$, $15 \mathrm{~mW} / \mathrm{cm}^{2}$ ); crosslinking the exposed areas. The remaining areas were washed away with developer. 
3. A polydimethylsiloxane (PDMS), purchased from Dow Corning GmbH, Germany, replica was made by mixing the PDMS precursor with its crosslinker (10:1) and pouring this mixture onto the silicon master. The PDMS was cured by baking at $80^{\circ} \mathrm{C}$ for 4-6 hours.

4. The PDMS mould was peeled off and inlet holes were made. The PDMS was then plasma bonded to a glass slide and tubing was connected to the resulting structure.

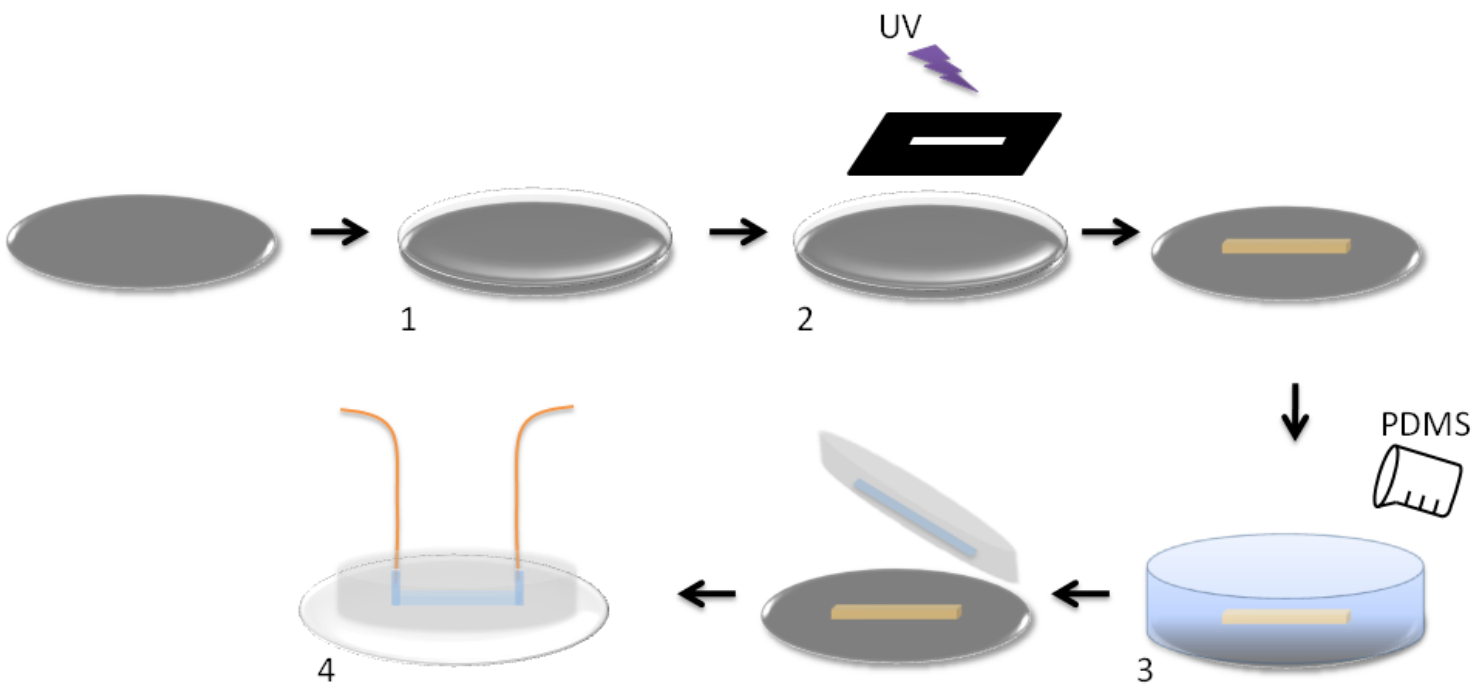

Figure 6.2: Steps followed for photolithography and soft lithography, numbers correspond to text.

PDMS is optically transparent, flexible, chemically inert, and surfaces can easily be functionalized. We found that trypanosomes injected into PDMS devices that had been cured at $80^{\circ} \mathrm{C}$ retained their vitality.

For flow velocities below $0.5 \mathrm{~mm} / \mathrm{s}$, hydrostatic pressure was used to control flow velocities. When higher flow velocities were required, fluid was injected and pumped through the channels using a custom made syringe pump with a step motor (Fig. 6.3). Previous calibrations have shown that fluctuations in flow velocity are minimal [81] and provide sufficient flow control for our studies.

Adhesion to the interior of the microfluidic channels can cause clogging and changes to flow field. To prevent such adhesion, before injection of the sample, the channel was flushed with 1\% Bovine Serum Albumin (BSA, purchased from Sigma Aldrich, Germany) for 30 minutes. Once BSA saturates the surfaces, unwanted adhesion can be minimized.

\subsubsection{Trypanosome preparation}

Trypanosomes were cultured in HMI9 complete medium [47; 46] supplemented with $10 \%$ fetal calf serum and collected at concentration of $2 \times 10^{5}$ cells $/ \mathrm{mL}$ as described in 


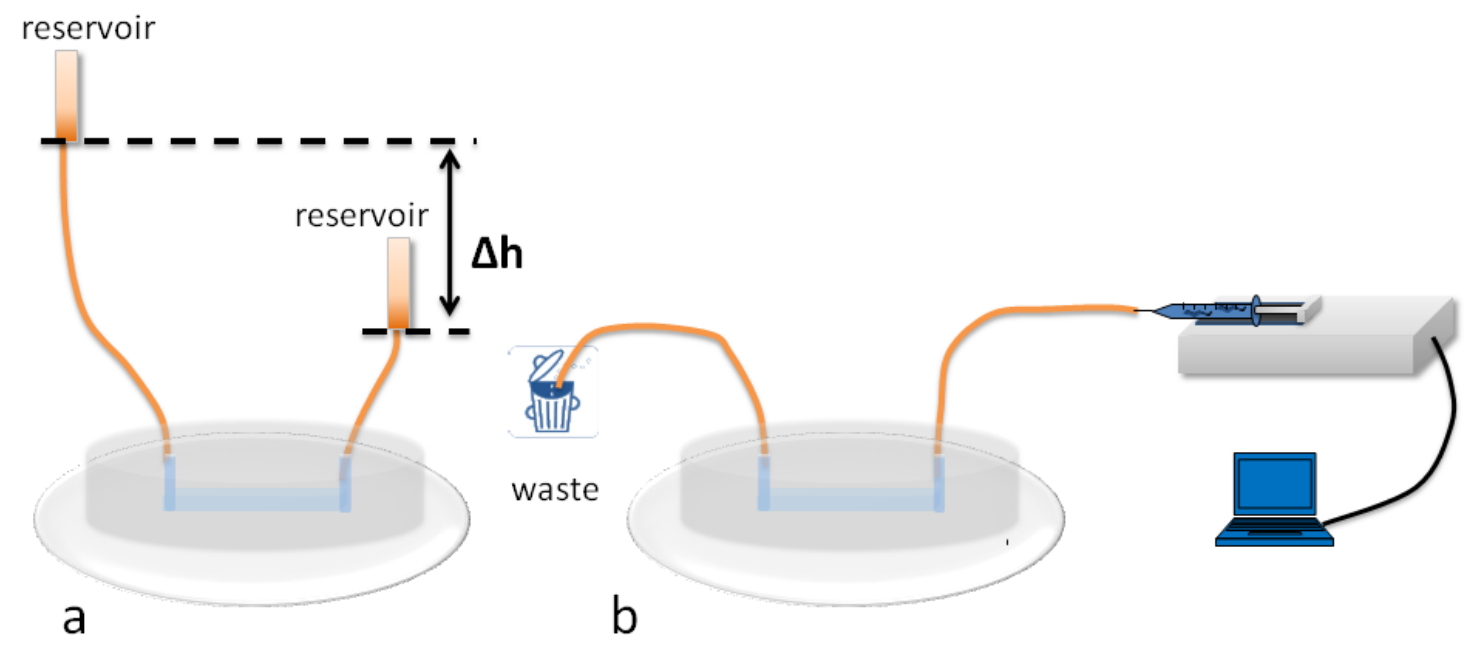

Figure 6.3: Methods for controlling flow velocities in microfluidic channels. a. using hydrostatic pressure and $b$. pumping system for higher flow velocities.

previous chapters. Cells were concentrated (but still kept sufficiently dilute to observe single cell dynamics in the microfluidic channel) and resuspended in fresh medium. For cell immobilization, trypanosomes were washed twice in trypanosome dilution buffer (TDB) (TDB; $5 \mathrm{mM} \mathrm{KCl}, 80 \mathrm{mM} \mathrm{NaCl}, 1 \mathrm{mM} \mathrm{MgSO}$, $20 \mathrm{mM} \mathrm{Na}_{2} \mathrm{HPO}_{4}, 2 \mathrm{mM} \mathrm{NaH} \mathrm{PO}_{4}$ ， $20 \mathrm{mM}$ glucose [pH 7.4])) and resuspended in TDB-2DG (5 mM KCl, $80 \mathrm{mM} \mathrm{NaCl}, 1$ $\mathrm{mM} \mathrm{MgSO}_{4}, 20 \mathrm{mM} \mathrm{Na} 2 \mathrm{HPO}_{4}, 2 \mathrm{mM} \mathrm{NaH}{ }_{2} \mathrm{PO}_{4}, 20 \mathrm{mM}$ 2-deoxy-D-glucose [pH 7.4])), incubated at $37^{\circ}$ for 10 minutes. This resulted in the cells being reversibly immobilized for up to 45 minutes. Both HMI9 complete medium and TDB have a viscosity similar to water, as measured by a Ubbelohde viscosimeter (VWR, Germany).

\subsubsection{Microscopy and image processing}

All experiments with microfluidic devices were conducted using a Zeiss Axiovert inverted microscope equipped with 100x air objective. Oil objectives were avoided to prevent any effects on flow velocity while shifting focus. Cells in flow were recorded with a PCO Sensicam and each movie frame was processed using a combination of Matlab (Mathworks), and Image Pro Plus (Media Cybernetics).

Movie frames were passed through a median filter, and converted to a black and white image (Fig. 6.5). The image processing toolbox of Matlab was used to detect trypansomes based on the size and aspect ratio of the object and the centroid was recorded. Subsequently, results were manually verified to avoid spurious detection.

A dilute concentration of polystyrene beads (Polysciences, GmBH Germany), $1 \mu \mathrm{m}$ in diameter was mixed with the sample before injection into channel. The motion of the beads was used to monitor the average flow velocities either by measuring the streak produced using long exposure times on the camera or by taking an average of the distances 


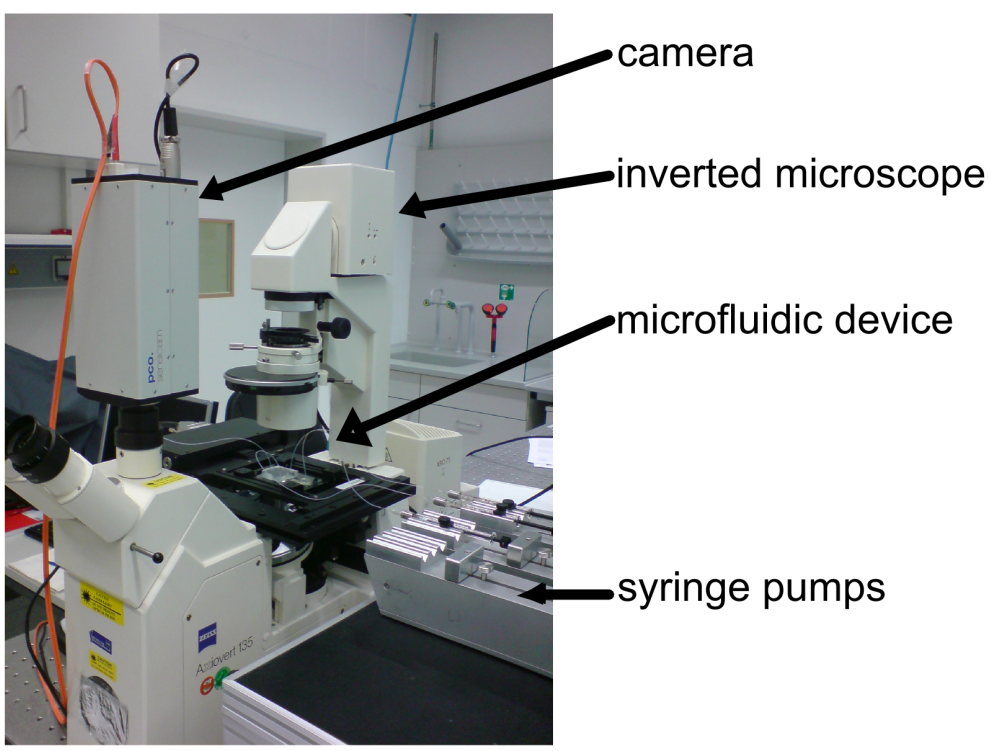

Figure 6.4: Experimental setup; microfluidic device attached to syringe pumps on a Zeiss Axiovert microscope.

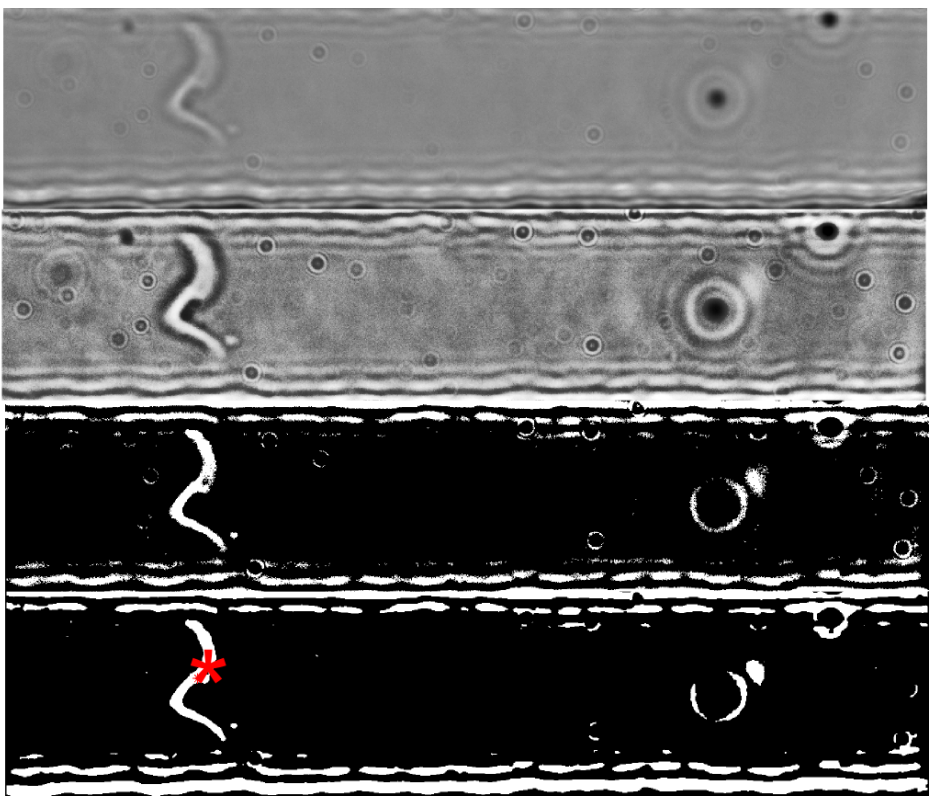

Figure 6.5: Image processing used to detect trypanosomes in microfluidic channel.

traveled by several beads from frame to frame in the channel.

Center of mass distributions were mirrored at channel center to improve statistics. Each distribution was constructed with a minimum of $\sim 150$ points.

'Sine wave' trajectories (discussed below), recorded over long distances were obtained using a 10x objective and tracking was performed manually. For higher flow ve- 


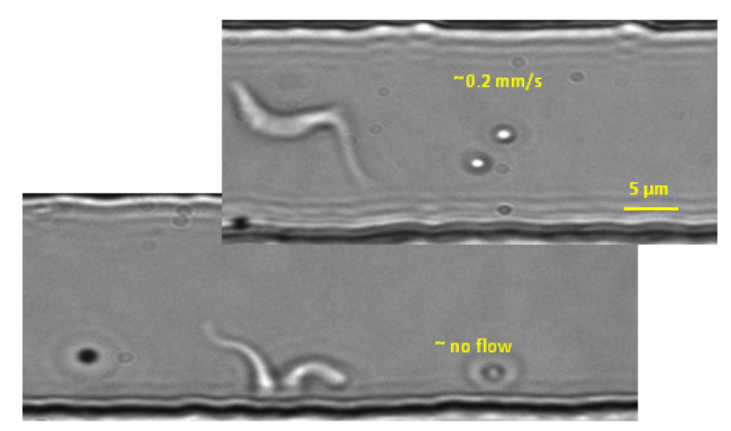

Figure 6.6: Typical images taken to record position of the cell within the channel

locities, observation times could be controlled by following the flow with a motorized stage at a matched velocity. Thus we could track the movement of the trypanosome in the $y$-axis, while it remained more or less constant in the $x$-axis. Sine wave fitting for oscillating trajectories was done by first smoothing the trajectory with 5-point adjacent averaging in Origin (OriginLab).

\subsection{Results}

In Fig. 6.7 a comparison of a live and dead cell swimming in such a microfluidic channel is shown at a flow velocity of $1.6 \mathrm{~mm} / \mathrm{s}$. This stroboscopic image, taken with fluorescently labeled cells, illustrates that a live cell is able to swim laterally across the width of the channel, while the dead cell is carried in the same streamline of the flow throughout the length of the channel. Fig. 6.7 suggests that despite flow velocities much higher than its own, the trypanosome continues to be an autonomous swimmer and it does not simply follow the flow profile. In contrast deformable vesicles, including red blood cells (RBC), have been shown to migrate toward the channel center due to the non-linear velocity gradient in Poiseuille flow [92: 93].

To investigate the behaviour of trypanosomes in flow, we begin with a symmetrical channel of equal height and width of $23 \mu \mathrm{m} \times 23 \mu \mathrm{m}$ (of the same length scale as the trypanosome body). As illustrated in Fig. 6.8, all observations were recorded only for trypanosomes in the middle plane $(\mathrm{z}=0)$ ensuring that they are subject to the same flow profile in the $z$ direction as seen in Fig.6.1. A suspension of trypanosomes in fresh culture medium was allowed to enter the channel. A very dilute concentration of polystyrene beads, $1 \mu \mathrm{m}$ in diameter, was included in the cell suspension to as tracers for flow. The symmetry of the height and width of the channel results in a parabolic velocity profile in all planes (see Fig. 6.1 for flow profile simulation). 


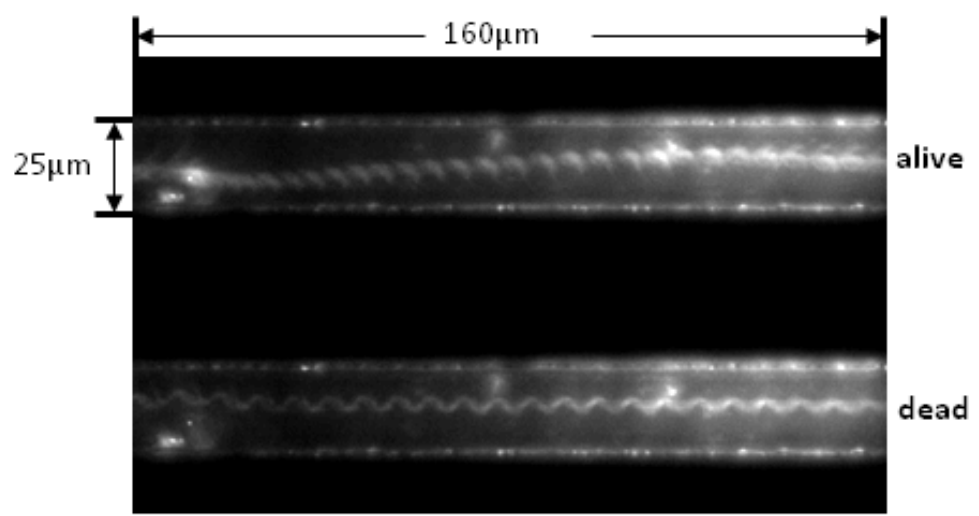

Flow velocity $1.6 \mathrm{~mm} / \mathrm{s} @ 300 \mathrm{~Hz}$

Figure 6.7: Despite flow velocities much higher than their own, live trypanosomes (top) are able to swim the width of the channel while a dead trypanosome stays in the same lateral position and get carried by the flow. Figure courtesy Eric Stellamanns [81].

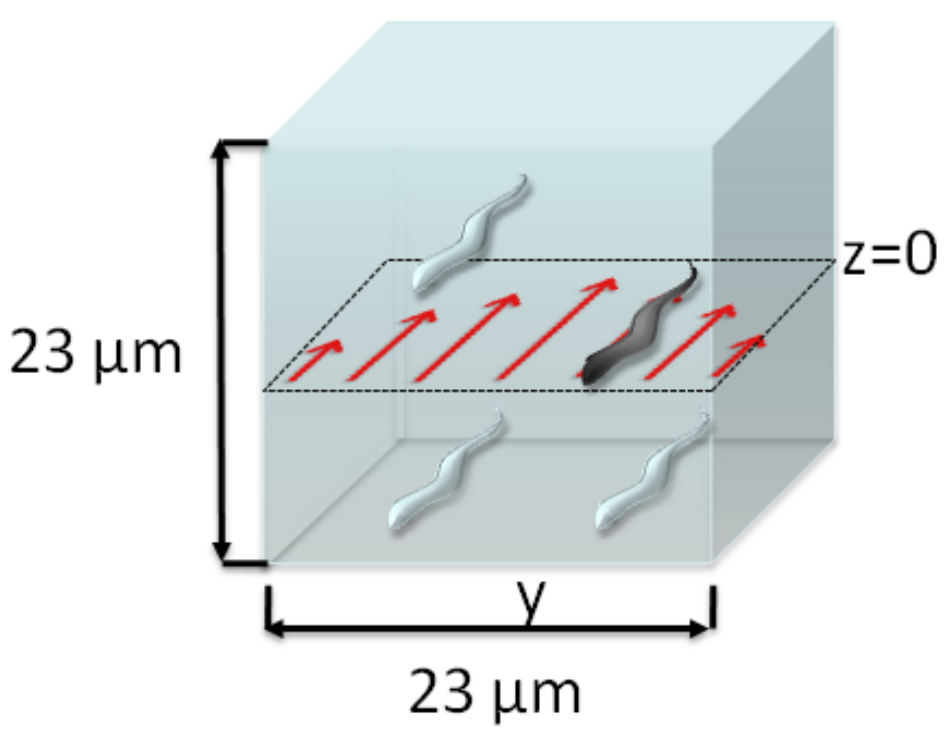

Figure 6.8: Only trypanosomes in the middle plane are monitored in a $23 \mu \mathrm{m} \times 23 \mu \mathrm{m}$ symmetric channel

\subsubsection{Sinusoidal trajectories in flow}

Intriguingly, when observed at lower magnification, allowing for longer observation times, we found that most trypanosomes swim upstream (with the flagellum-end leading) from one wall to another in a 'sine wave trajectory' when $U_{\text {ave }}<0.4 \mathrm{~mm} / \mathrm{s}$, as in Fig. 6.9. As Fig.6.10illustrates, the observed trajectories are well fitted by sine waves.

Note that the trajectories in Fig. 6.10 appear jagged due to the fast distortions of 


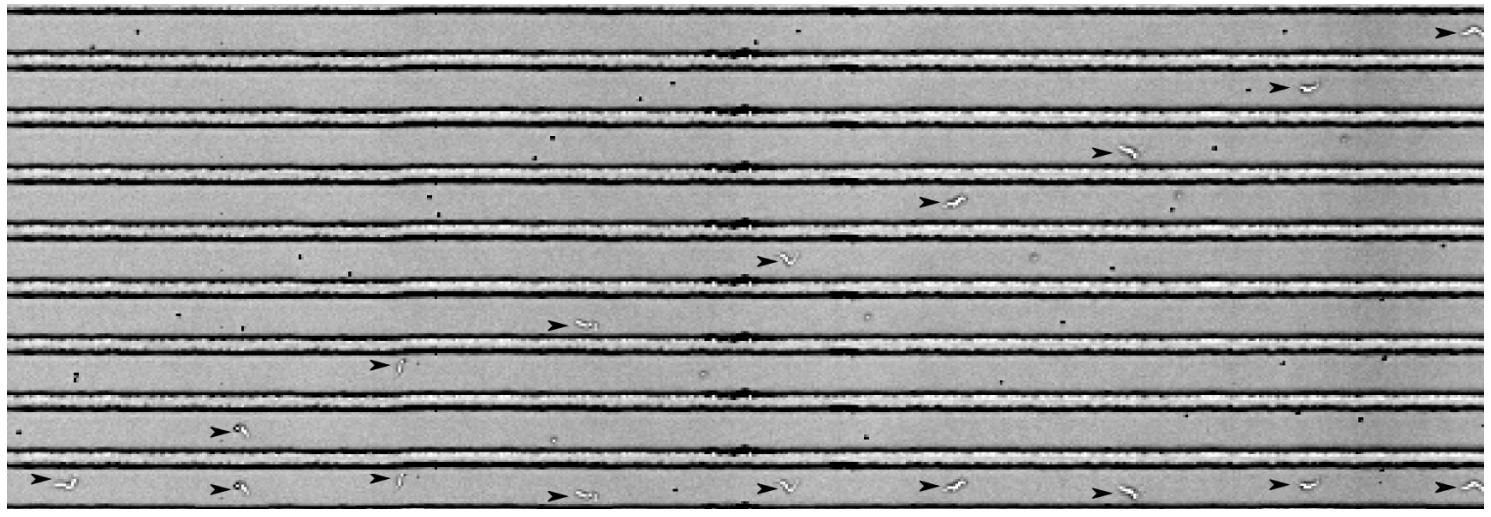

Figure 6.9: Montage of a cell moving in pressure driven flow through a microfluidic channel. The cell (black arrow) moves in a sinusoidal trajectory within an upstream orientation. Channel width is $23 \mu \mathrm{m}$. Lowest panel shows the overlay of all.

a

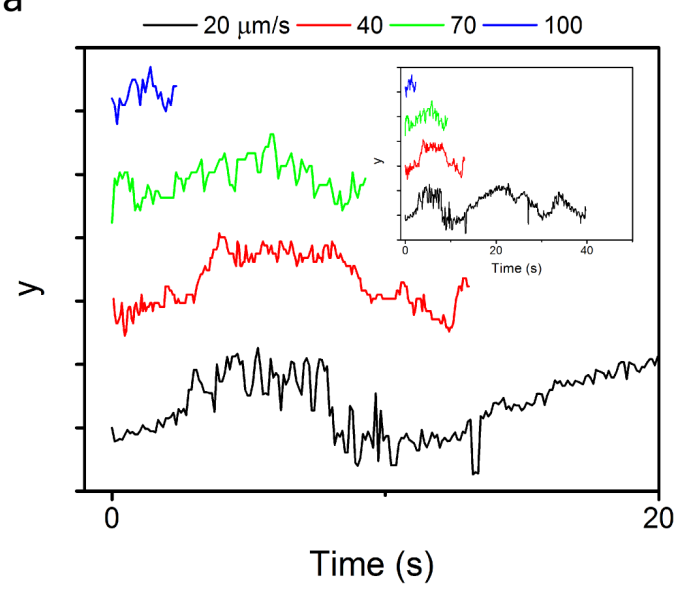

b

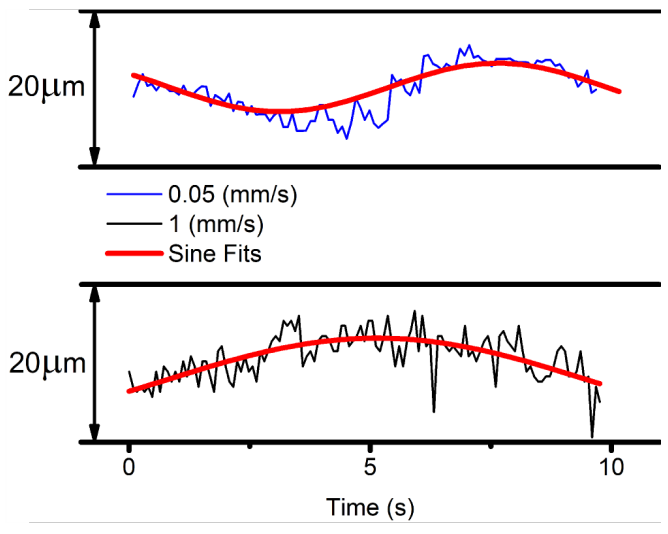

Figure 6.10: a. Illustrative trajectories (y-coordinate) of trypanosomes at different flow velocities (each tick on the y-axis is $10 \mu \mathrm{m}$ ). Higher flow velocities reduce the time in which the cells can be tracked (shown in the inset). $\mathrm{b}$. The trajectories are well described by sine waves.

the trypanosome body during swimming as discussed in see Chapter 4. Trypanosomes swimming with a downstream orientation do not exhibit such oscillatory paths. At higher flow velocities, trypanosomes quickly swam out of the field of view of the camera. Trajectories at a range of flow velocities were fitted to sine waves and the frequency and amplitude of oscillations are shown in Fig. 6.11 as a function of flow velocities. Increasing velocities resulted in increasing frequency and decreasing amplitude of the trajectories.

The oscillations in Fig. 6.10 could be attributed to the shear force pushing the trypanosome away from the wall; at a critical point, the trypanosome reorients toward the channel center. As it approaches the opposing wall, the shear generated at this wall may then act to push the trypanosome away again and so on, resulting in the observed si- 

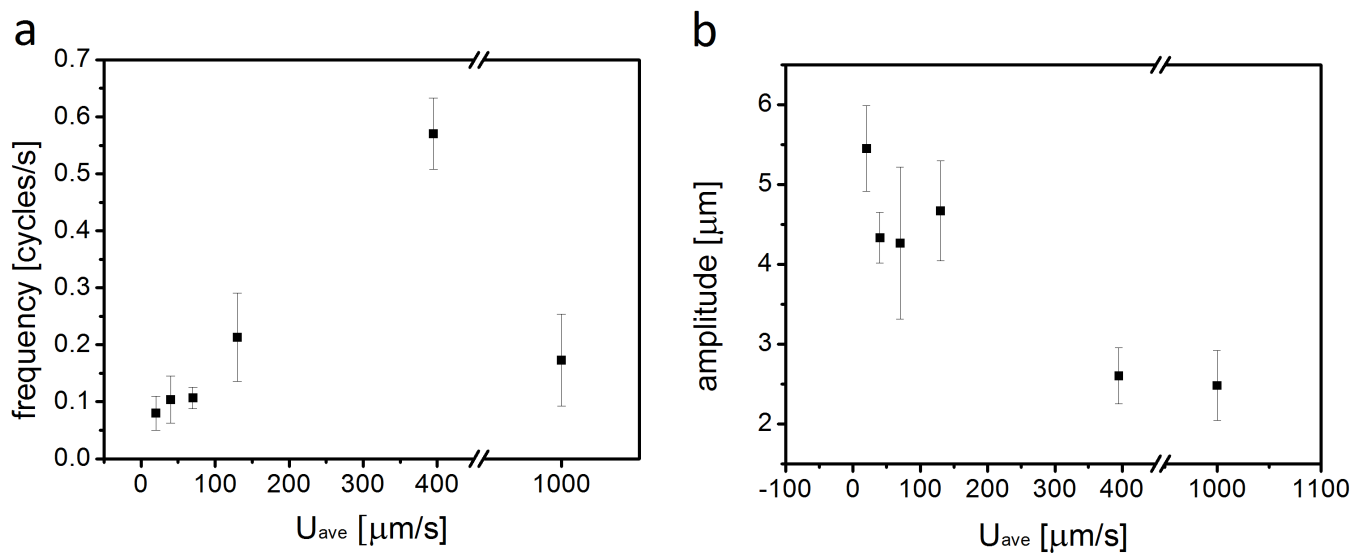

Figure 6.11: The frequency of the oscillations (a) increases with flow velocities while the amplitude (b) decreases. At least three trajectories were measured at each velocity

nusoidal trajectories. An increase in the shear rates due to higher flow velocities would force the trypanosome away from the wall sooner thus narrowing the trajectory (decrease in amplitude), and increasing the frequency of the oscillations.

Note that the trends observed here are seen for flow velocities that are up to five times the velocity of the trypanosome itself. As the flow velocity was increased further, sine wave trajectories were less frequent and tumbling motion of the cell dominated (see also below the discussion cell orientation). To capture trajectories at higher flow velocities, a motorized stage was used to follow the channel at the corresponding velocity; thus the trypanosome position in the lateral (y-coordinate) axis relative to the channel wall was recorded (Fig. 6.10p, $1.0 \mathrm{~mm} / \mathrm{s}$ ). At $1.0 \mathrm{~mm} / \mathrm{s}$, we found that some cells still exhibit the oscillatory motion, though cell orientation was not distinctly upstream. At such a high relative flow velocity other factors such as a lift force and enhanced cell tumbling may come into play.

\subsubsection{Center of mass distribution}

We pursue these observations by exploring the effects of flow velocity on lateral cell positioning within the same experimental setup. The center of mass of cells (at $z=0$ ) was semi automatically detected as described in the image processing section above and used to construct the center of mass distribution shown in Fig. 6.12a along the channel (ydirection). For consistency the center of mass was recorded within a $2 \mu \mathrm{m}$ interval for all distributions shown below. In the absence of flow, cells are, for the most part, at the channel walls. This result is in agreement with studies of sperm cells [94], and E.coli swimming between two parallel plates [90: 87]. Indeed, theoretical work suggests that hydrodynamic flow fields which are generated by the flagellar beat of the sperm 'capture' the sperm near the walls of the container [94: 95]. At a low flow velocity of $0.1 \mathrm{~mm} / \mathrm{s}$ try- 
panosomes swim mostly in the center of the channel. As we will see below at this range of flow velocity, self propulsion may dominate the dynamics. As the velocity is increased to $0.4 \mathrm{~mm} / \mathrm{s}$, the distribution of cells in the center tends to flatten slightly, and as the velocity is further increased to $1.0 \mathrm{~mm} / \mathrm{s}$ the distribution is further flattened and broadened thus more cells are found closer to the channel walls. Given the no-slip boundary condition, the fluid velocity at the channel wall is zero possibly allowing the trypanosome to avoid the sweeping effects of velocity fields and thus more autonomy to explore their boundaries once they are at the walls of the channel (or blood vessel). The expected depletion layer of at least $2.5 \mu \mathrm{m}$ (due to the average diameter of a trypanosome) is actually at least twice as much for all the investigated flow velocities suggesting the presence of an additional influence causing the cell to move away from the wall. Both the self propelling nature of the trypanosome and a shear induced lift may contribute to the depletion layer. Nevertheless, changes in velocity do appear to have an effect on the trypanosomes' ability to position itself in the channel.

Studies of semi-flexible and rigid polymers in flow have shown a migration away from the boundaries and toward the center of the channel [96; 97; 98; 99; 100]. Hydrodynamic interaction at the boundaries due to increased shear lead to the observed depletion. The migration is not significantly influenced by the flexibility of the polymers [96], however the effect is enhanced with increasing flow due to a corresponding increase in shear stress at the walls [99].

Shear induced migration could explain results the peak in the distributions shown in Fig. 6.12. However, in contrast to the studies of polymers in flow, increased flow velocities do not enhance the depletion. Unlike the polymer studies in [96; 97; 98], the width of the channel we study is of the same order of magnitude as the object in flow. Thus the shear gradient becomes steeper with increases in flow velocity and uneven forces acting on the length of the cell body (see Fig. 6.18) may cause a cell to tilt more frequently resulting in a broader distribution. At low flow velocities the cells are able to align preferably with the streamlines (i.e parallel to the channel walls) resulting in a narrower center of mass distribution. Indeed in [91] semi-flexible polymers in microchannels of the same length scales were found to be highly influenced by the shear gradient.

To verify whether the self propelling nature of the trypanosome is the key to the observed trend, or if the shape, size and density of the cell itself are sufficient we used immobilized trypanosomes. Dead cells (killed either by heating, or ethanol treatment) very quickly start to lyse and are therefore unsuitable. Standard cell fixation techniques such as methanol or paraformaldehyde treatment do not lyse the cell, but cross link the cell membrane and cytoskeleton thus increasing cell stiffness which in turn may influence behaviour in flow. To address these issues, we immobilize trypanosomes by replacing the glucose in TDB by 2-deoxy-D-glucose thus depleting the source of ATP in the environment. In such a suspension, cells were reversibly immobilized for up to 45 minutes, and gave us access to the inanimate entity we were seeking. 2-deoxy-D-glucose (2DG) is a 

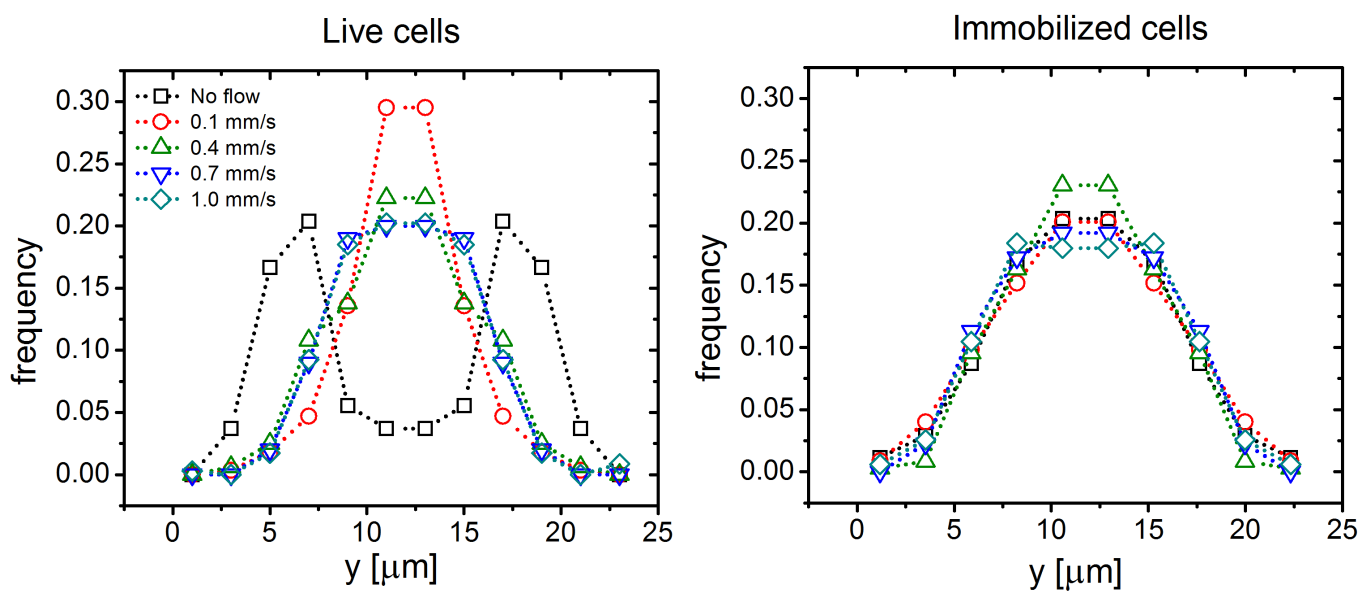

Figure 6.12: Center of mass distribution of cells in a $23 \times 23 \mu \mathrm{m}$ channel. a. Live cells. $b$. Immobilized cells. Results are mirrored at the center of the channel to increase statistics. Distributions are normalized by the total number of cells recorded.

stable glucose analogue that is actively taken up by glucose transporters but cannot be fully metabolized and thus blocks the glycolytic pathway [101; 102].

We collect again the center of mass distribution with the immobilized trypanosomes along the width of the channel at increasing velocities as seen in Fig. 6.12. The most striking difference between active and immobilized trypanosomes is seen in the absence of flow. While live cells tended to swim close to the channel walls, immobilized trypanosomes were more likely to be found away from the walls. Near the walls the immobilized cells experience a reduced diffusivity, hence the tendency migrate away from the wall. We found a similar trend in the center of mass distribution at all flow velocities for live and immobilized cells, ranging from $0.1 \mathrm{~mm} / \mathrm{s}$ to $1 \mathrm{~mm} / \mathrm{s}$; a depletion layer of $\sim 5$ $\mu \mathrm{m}$, followed by a steady increase in the number of cells toward the channel center and a small plateau at the center. Since the cells are immobilized they are not able to align themselves to the streamlines rendering them more susceptible to velocity gradients even at low flow velocities. This is illustrated in the measurements of the end to end distance of the cells below.

\subsubsection{Cell elongations}

The effect of flow on the body of trypanosome is also characterized by the overall elongation of the body. In Fig. 6.13 histograms of the end to end distance (distance from the flagellum tip to the base of the cell body - as in Chapter 3) of the cell for both live and immobilized cells show us two important aspects of cell motility in flow. Increasing flow velocities, leading to increased shear stress, results in a more elongated cell shape for immobilized cells in agreement with simulation for semi-flexible polymers [96]. In the absence of flow, live cells have the same end to end distribution as immobilized cells. 

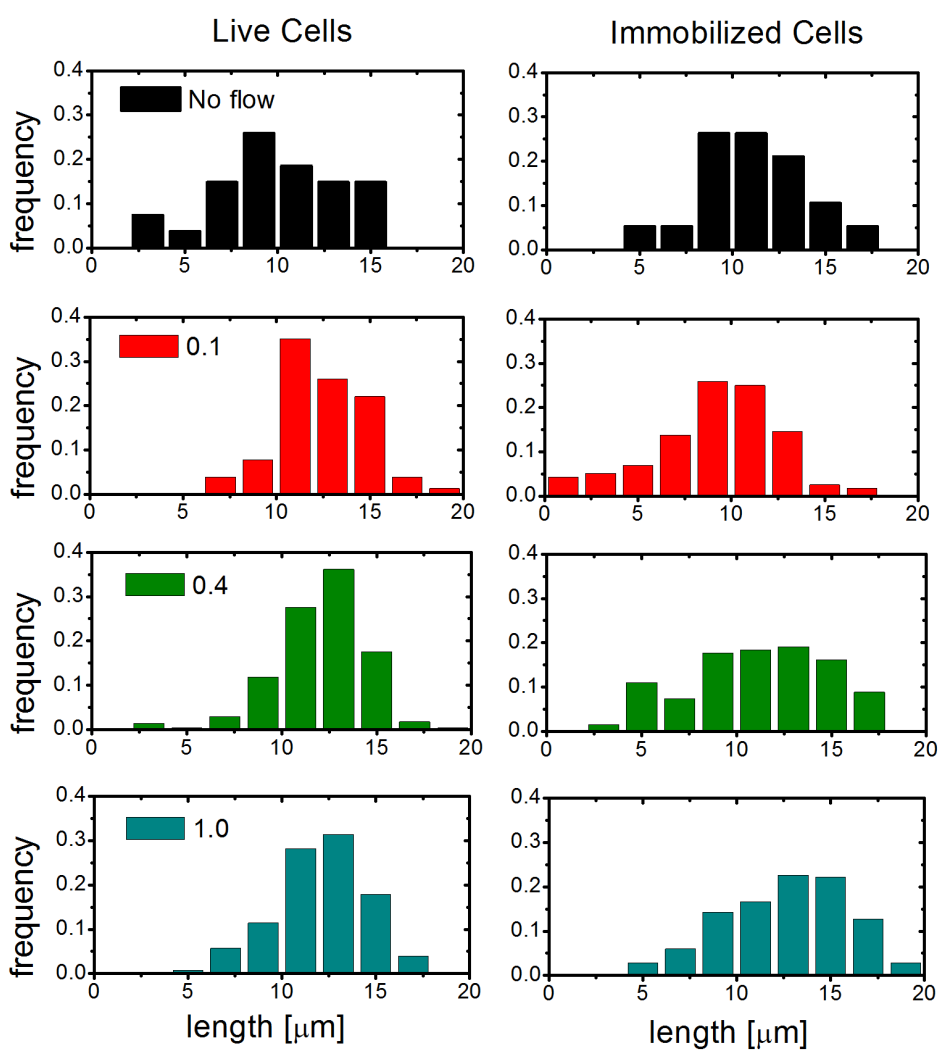

Figure 6.13: Without flow, both live cells and immobile cells exhibit the same average end to end distance. While live cells show the same average elongation at all measured flow velocities, immobilized cells appear to stretch in response to increased shear.

Upon inducing flow, the end to end distance of live cells appears to have little dependence on flow velocity for live cells showing that even at $1.0 \mathrm{~mm} / \mathrm{s}$ trypanosomes are able to produce body distortions as shown in Fig. 6.14. We observed that within the range of flow velocities that we examined that while live cells are not straightened, immobilized cells begin to flatten with a linear dependence of flow velocity. Differences in cell length are assumed to be equivalent in all populations and are therefore averaged out for data sets collected at each velocity.

We can therefore liken the cell body to a spring dumbbell illustrated in Fig. 6.15 following Hooke's law, with the drag force acting on the cell body due to fluid flow acting as the stretching force.

The drag force causing cell stretching is a function of the velocities $U_{1}$ and $U_{2}$ at each end of the dumbbell and is given by:

$$
F_{d}=\gamma\left|\mathbf{U}_{2}-\mathbf{U}_{1}\right|
$$

We take a simplified approach with $U_{2}-U_{1} \sim U_{\text {ave }}$. Taking the radius of the beads of the dumbbells to be $3 \mu \mathrm{m}$, we calculate $\gamma$ from Eq. 3.7 as $5.7 \times 10^{-8} \mathrm{~kg} / \mathrm{s}$. Eq. 6.3 yields 

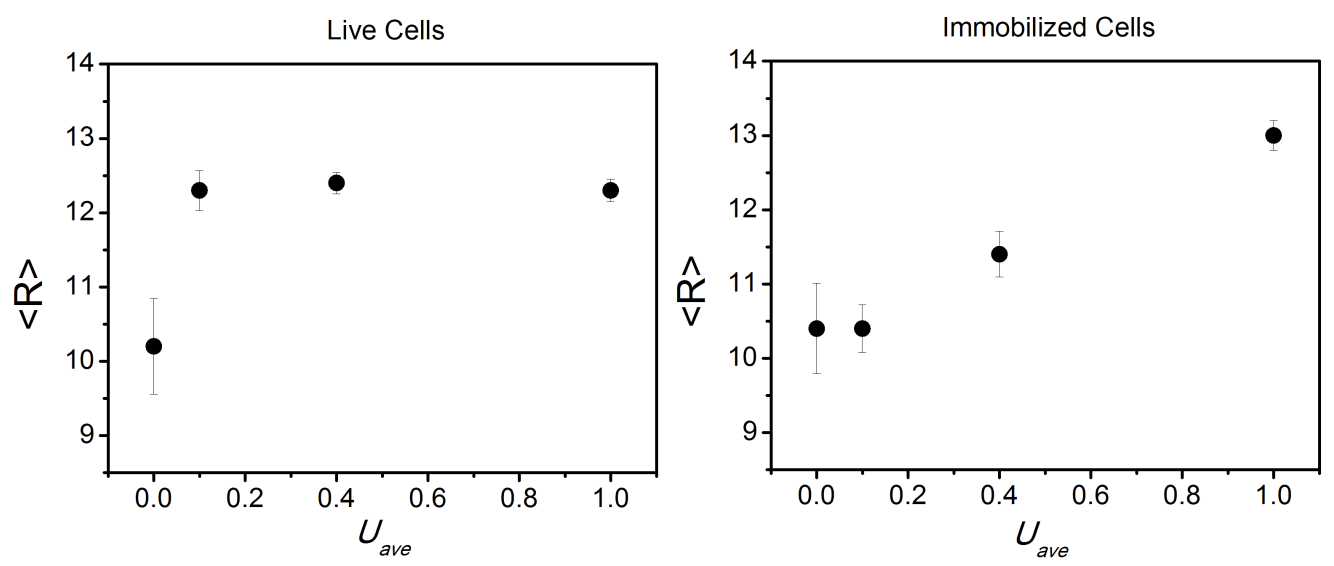

Figure 6.14: Mean end-to-end distance $[\mu \mathrm{m}]$ vs. average flow velocity $[\mathrm{mm} / \mathrm{s}] U_{\text {ave }}$ for live and immobilized cells.

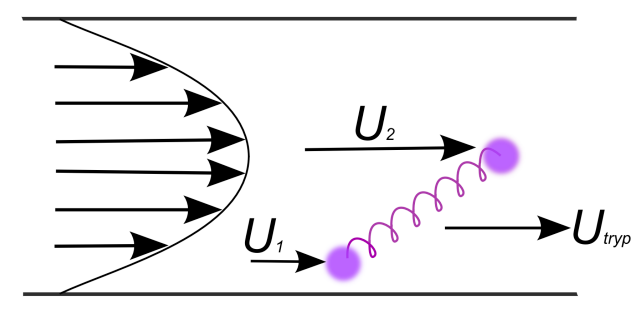

Figure 6.15: Trypanosome cell body as a dumbbell in a parabolic flow profile.

drag forces in the $\mathrm{pN}$ range and can be plotted versus the cell stretching, given by $\Delta x=$ $\langle R\rangle-\left\langle R_{e q}\right\rangle$ where $R_{e q}$ is the end to end distance in the absence of flow, also yields a linear relationship shown in Fig. 6.16 .

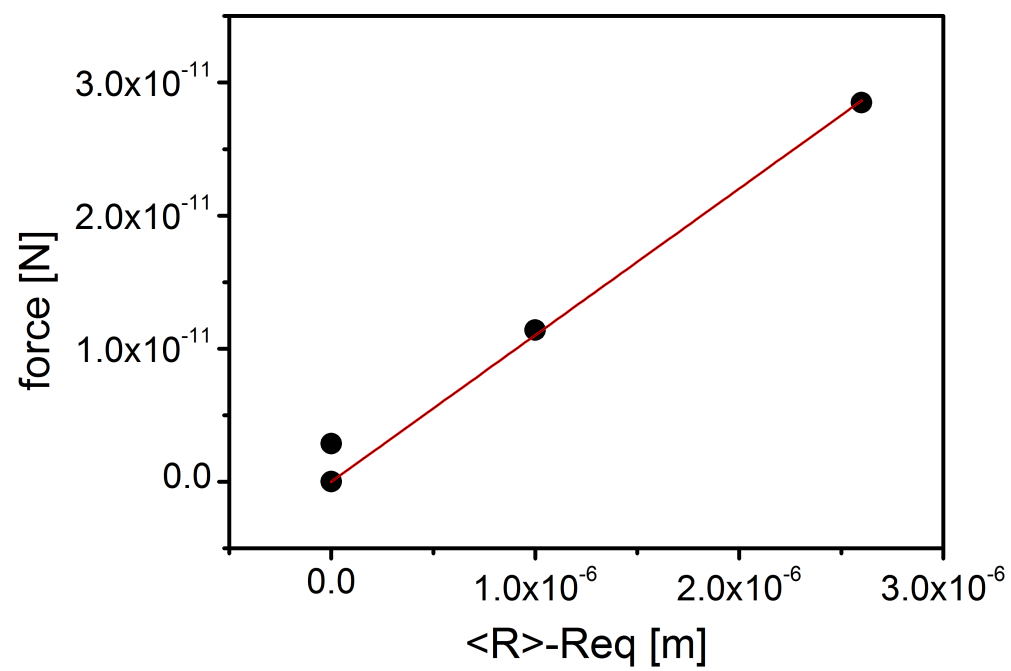

Figure 6.16: Drag force resulting from fluid flow plotted as a function of cell stretching 
Cell stretching, taken as a spring, may therefore be described by Hooke's law where $F=-k \Delta x$. The slope of the line in Fig. 6.16 gives us the value of $1.1 \times 10^{-5} \mathrm{~N} / \mathrm{m}$ for $k$. Recent experiments on Vorticella convallaria found that the cell body is extended in microfludic flows with drag forces on the order of $50 \mathrm{nN}$ [103] and validated the use of flow-induced load to measure cell stiffness. Elongated cells comprised of microtubule cytoskeletons, such as trypanosomes, have not been studied in this context. Cells that are 10-100 fold wider than trypanosomes have been reported to have stiffnesses on the order of $10^{-3}$ to $10^{-4} \mathrm{~N} / \mathrm{m}$ - reflecting the cross sectional area $A$ of the cell $[104 ; 103 ; 105 ; 106 ; 107]$ (the stiffness depends as $k=E A / L$, where $E$ is the elastic modulus and $L$ is the length of the cell). Furthermore, the obtained value is $\sim 2$ fold greater than that of reported values for red blood cell stiffness, $\sim 6 \times 10^{-6} \mathrm{~N} / \mathrm{m}$ [108; 109]. Thus, we find that the stiffness estimate for trypanosomes found here is reasonable.

\subsubsection{Cell orientation}

Since the velocity gradient is on the length scale of the cell itself, each segment of the body may experience a different drag force, therefore we investigated cell orientation as function of velocity and channel position.
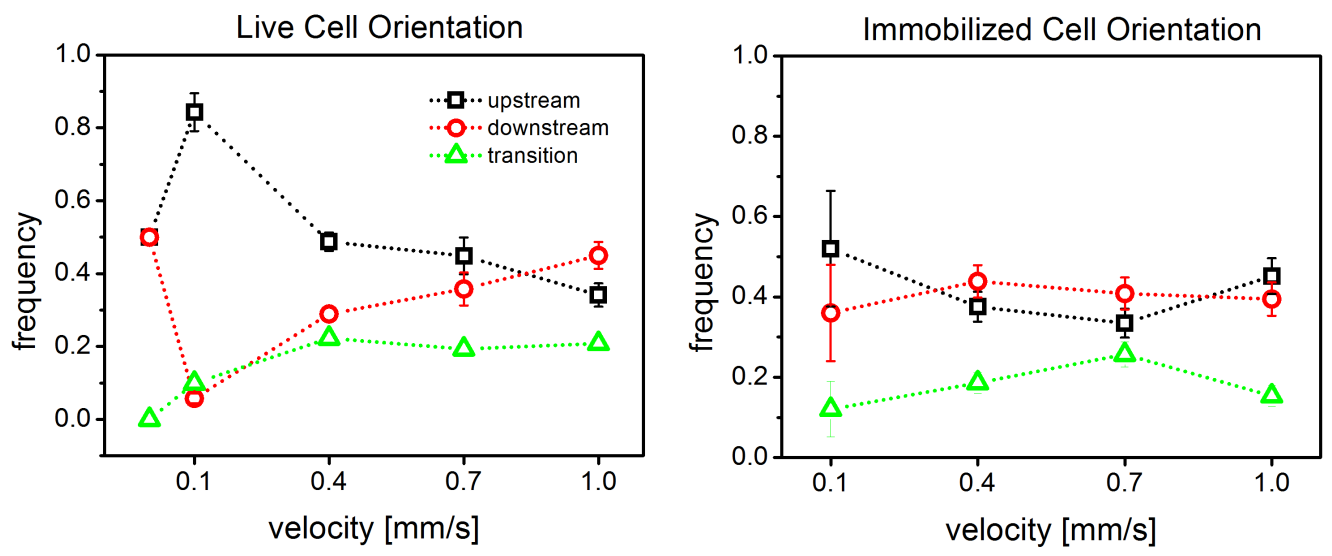

Figure 6.17: Cell orientation relative to flow for live and immobilized cells. For live cells in the absence of flow an arbitrary direction is chosen for the 'flow'. Note that immobilized cells tend to sediment therefore orientation is not shown.

The frequency of upstream or downstream swimming is summarized in relation to flow velocity in Fig. 6.17. We found that at low flow velocities $(0.1 \mathrm{~mm} / \mathrm{s})$ most cells swim upstream (with the flagellum end leading) suggesting that the trypanosomes swim toward the oncoming flow field (upstream). Depending on the flow velocity there may still however be a net cell body movement downstream. The orientation preference is lost once the velocities increase further and indeed begin to display a small tendency to swim downstream at $1 \mathrm{~mm} / \mathrm{s}$ flow velocity. Cells that were tumbling to recover one of the two orientations were recorded as having transition orientation (green in Fig. 6.17). 
Remarkably immobilized cells do not exhibit any significant orientation preference across the velocity spectrum (see Fig. 6.17p) suggesting that self propulsion plays an important role in orientation, especially in the lower range of flow velocities.

At low flow velocities the tapering shape results in an increased drag toward the base of the cell likely forcing the cell to orient upstream. The propulsive force of the cell dominates and allows it to swim upstream. As the shear gradient increases (red in Fig. 6.18) and the corresponding increase in drag dominates the motion of the trypanosome resulting in the observed tumbling motion of the cell seen at higher flow velocities.

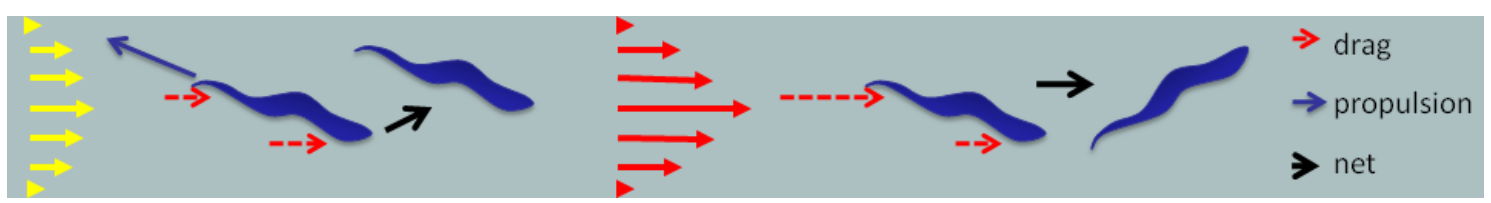

Figure 6.18: At low flow velocities (yellow), the tapering shape and self-propulsion dominate, as the velocity gradient increases (red) the corresponding increase in drag dominates the motion of the trypanosome and results in a tumbling motion.

A more detailed investigation of cell behaviour at the walls was achieved using an orientation order parameter given by:

$$
S=\left\langle\frac{3 \cos ^{2} \theta-1}{2}\right\rangle
$$

Here $\theta(-90<\theta<90)$ is the angle of the end-to-end vector relative to the flow direction (Fig. 6.19).
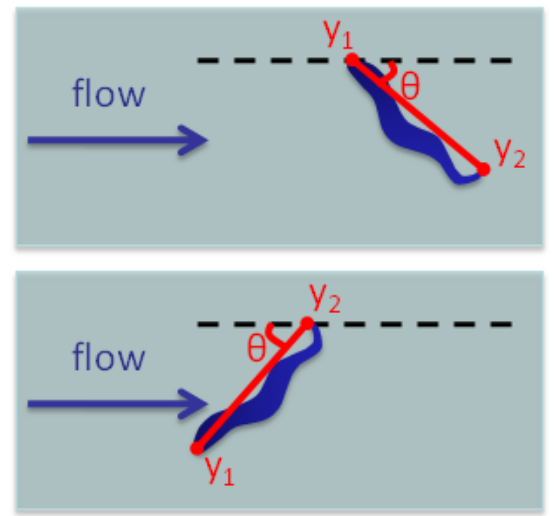

\begin{tabular}{l|l|l|l}
\hline & $\begin{array}{l}\text { Random } \\
\text { Orientation }\end{array}$ & $\begin{array}{l}\text { Parallel } \\
\text { to Wall }\end{array}$ & $\begin{array}{l}\text { Normal } \\
\text { to Wall }\end{array}$ \\
\hline$<\cos ^{2} \theta>$ & $1 / 3$ & 1 & 0 \\
\hline$S$ & 0 & 1 & $-1 / 2$ \\
\hline Schematic & & flow & \\
& &
\end{tabular}

Figure 6.19: Angle $\theta$ used to calculate the orientation order parameter $S$. The value of the $S$ gives an indication of how the cell is aligned with the walls.

When cells are aligned to the channel wall, $S=1$, while random orientation to all possible angles yields $S=0$ and an orientation perpendicular to the wall yields $S=-1 / 2$. Note that the angle $\theta$ is defined as positive if the endpoint corresponding to $\mathrm{y}_{1}$ (illustrated in Fig. 6.19) is closer to the wall than $y_{2}$. Therefore, while cell bending is quantified in 
Fig. 6.13 and direction (upstream or downstream) is represented by Fig. 6.17/respectively, the orientation parameter $S$ is a measure of cell tilting with respect to the wall.

In Fig. 6.20, the orientation parameter $S$ is presented for both live and immobilized cells (red and black) as a function of channel position for different flow velocities. In both live and immobilized cells, the orientation order parameter increases close to the walls and away from the channel center - yielding a small minimum at the center of the channel. This trend seems to be more prominent with live cells. This drop in orientation at the center may be attributed both to the minimum in shear and reduced steric hindrance, allowing cells to orient, on average, randomly. Immobilized cells appear to be less oriented to the flow which can be attributed to their tendency to tumble in flow.
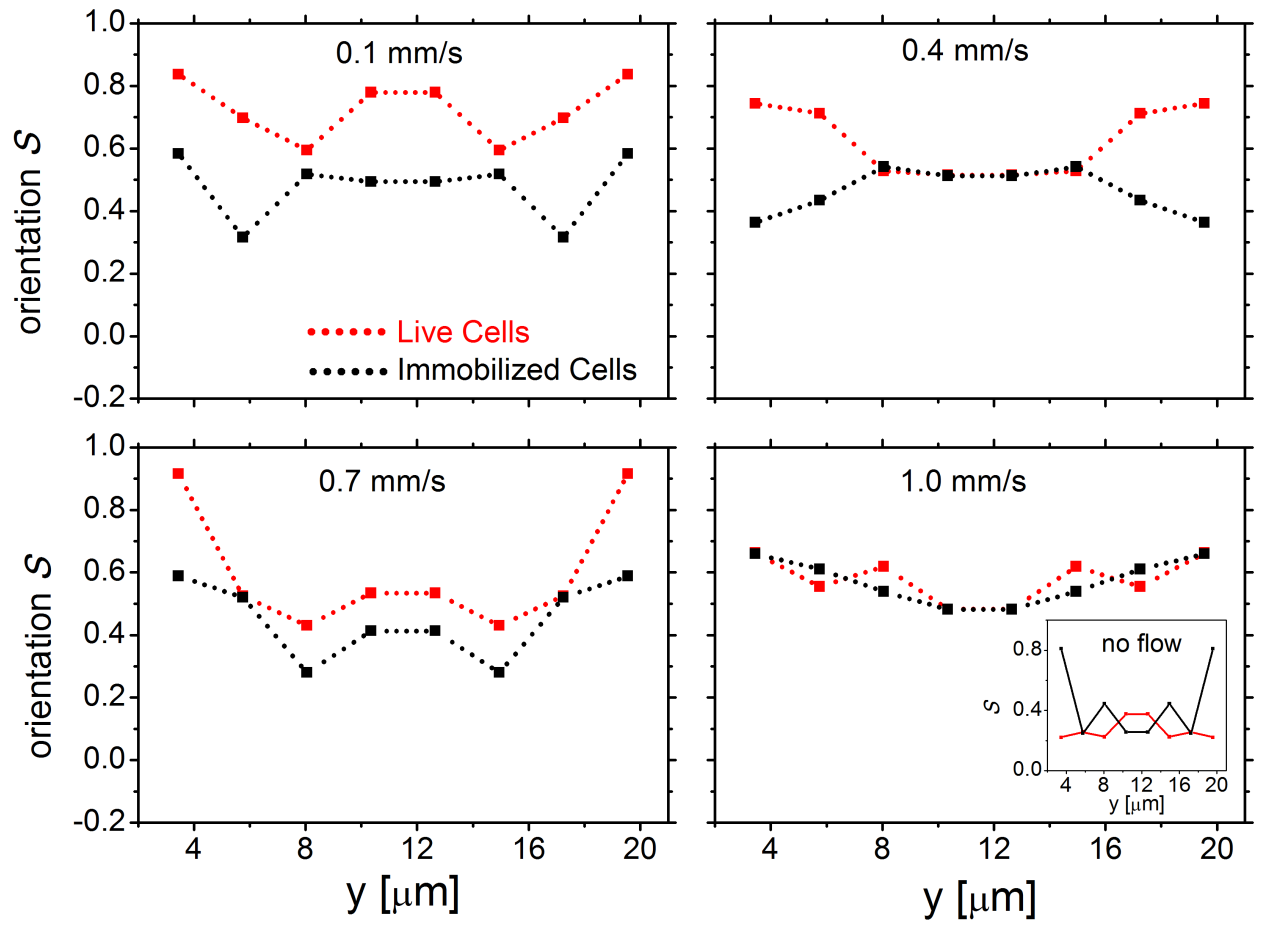

Figure 6.20: Orientation order parameter for live (red) and immobilized cells (black), shown for a range of velocities. Results are mirrored at the center for improved statistics. The inset shows the orientation for cells without flow; here the orientation is measured by simply choosing a 'direction for flow'. Note that immobilized cells tend to sediment in the absence of flow, but an orientation is nevertheless measured for comparison.

In the absence of flow, we note that as live cells appraoch the walls they become highly aligned to the walls. Immobilized are randomly oriented across the channel. The most dramatic difference in orientation parameter between live and immobilized cells in flow is seen at the lowest velocity. As the flow velocity is increased up to $1 \mathrm{~mm} / \mathrm{s}$ we see that the orientation between live and immobilized cells converges. Results presented earlier sections of this chapter also showed that at 0.1 and $0.4 \mathrm{~mm} / \mathrm{s}$ self propulsion dominates dynamics in flow, but at the higher velocities shear forces dominate. 


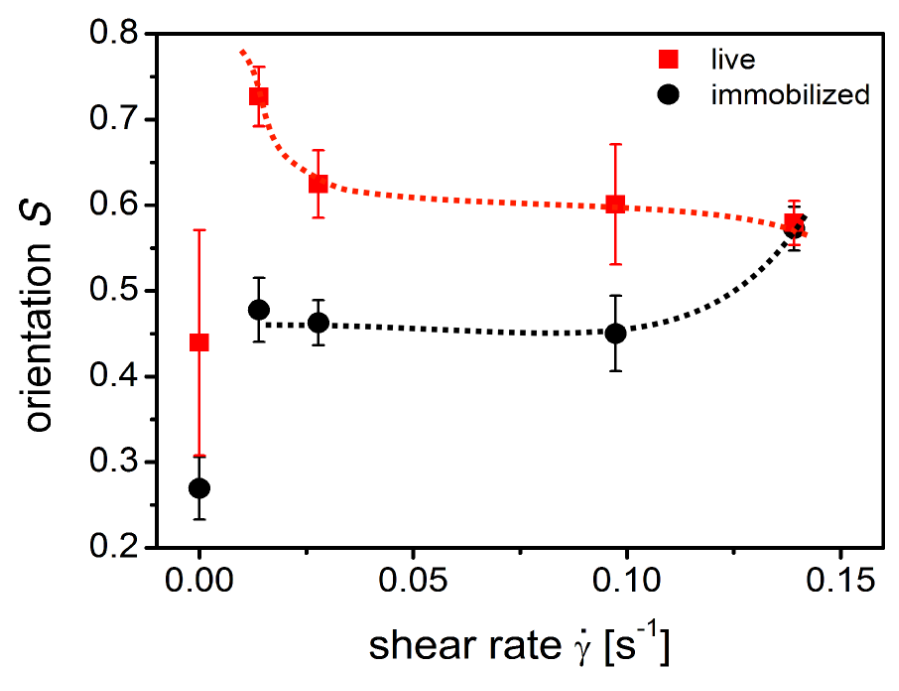

Figure 6.21: Mean orientation order parameter (for $y=2$ to $y=20$ ) for live and immobilized cells, shown as a function of mean shear rate. Dashed lines are a guide to the eye.

The effect of shear rate on orientation is shown in Fig. 6.21. In the absence of flow (and therefor shear), live cells are more aligned than immobilized cells. We observe a distinct effect at low flow velocities, showing clearly that live cells are well aligned to the boundaries $(S=0.73)$ at the lowest measured shear rate. Further increases in shear rate reduces the mean cell orientation.

Contrastingly, immobilized cells exhibit a much lower orientation $(S=0.48)$ at low shear which shows an increasing trend as shear rate is increased. At the highest shear rate (corresponding to $U_{\text {ave }}=1.0 \mathrm{~mm} / \mathrm{s}$ ), we observe clearly that the effect of self propulsion is negligible since live cell orientation and immobilized cell orientation are equivalent.

\subsection{Summary}

Trypanosome swimming was characterized in microfluidic channels with flow velocities ranging from 0.1 to $1.0 \mathrm{~mm} / \mathrm{s}$. In the lower flow velocity range trypanosome orient themselves upstream and oscillate from one channel wall to another following sinusoidal paths. Further increases in flow velocity resulted in more tumbling cells. In the absence of flow, most cells were found at channel walls, but once flow was induced cells were, for the most part, found at center of the channel. Within the flow velocity range, live cells retain their ability to bend and distort their bodies. Immobilized cells begin to stretch as a linear function of flow velocity and could be used to derive cell stiffness. Comparison with immobilized cells showed that self propulsion dominates cell dynamics at mean flow velocities up to $0.1 \mathrm{~mm} / \mathrm{s}$. 



\section{Motility in biomimetic flow}

In the blood stream of the mammalian host, trypanosomes are confronted with a crowded environment of strong shear stresses. Blood flow velocities range from $\mathrm{mm} / \mathrm{s}$ in capillaries to $\mathrm{m} / \mathrm{s}$ in the aorta - orders of magnitude higher than the average swimming speed of the trypanosome. Despite such high flow velocities, trypanosomes are able to invade and populate tissue spaces, including the blood brain barrier, from the blood vessels (Fig.7.1). Thus suggesting that trypanosomes are preferentially positioned near the wall of a blood vessel. In contrast, non-self propelling deformable vesicles, including red blood cells and lipid vesicles, are known to migrate away from vessel walls in a bounded flow.

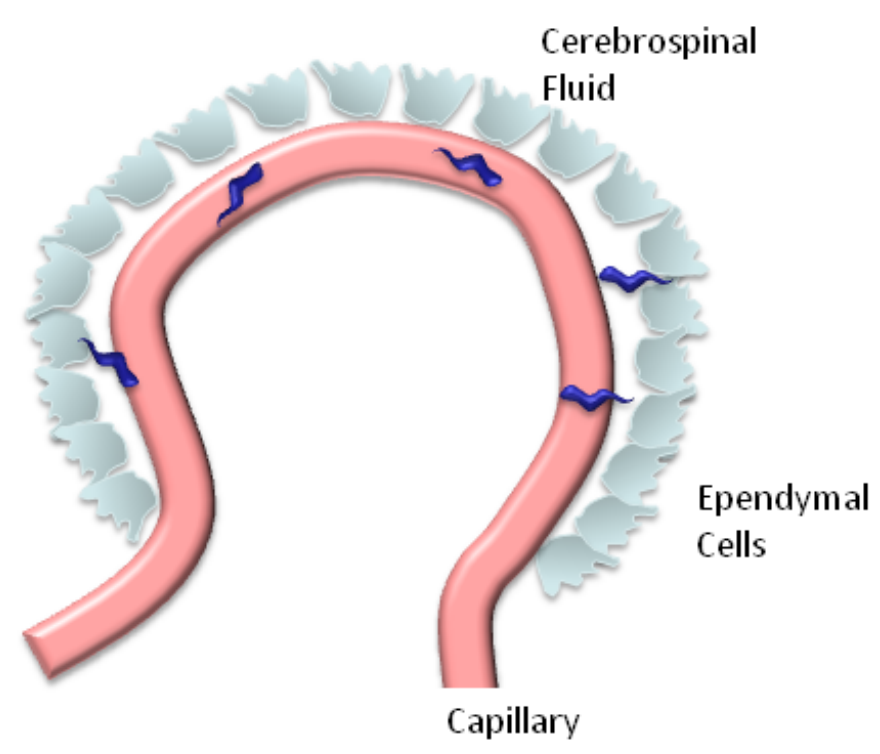

Figure 7.1: Trypanosomes swim in capillaries and enter the CNS through the blood brain barrier

In this chapter we begin by explaining basic principles of blood flow. We then we build on the work presented in Chapter 6 by studying trypanosome motility in blood 
flow and present our studies towards understanding how they are able to occupy, and eventually penetrate membrane barriers while having to navigate through high flow velocities in the blood stream.

\subsection{Blood flow}

Blood is comprised of erythrocytes (red blood cells, RBCs), leukocytes (white blood cells, WBCs), platelets and plasma - the suspending fluid (see Fig. 7.2). The main role of blood is to transport nutrients and waste, protect during inflammation/infection and regulate balance within the body.

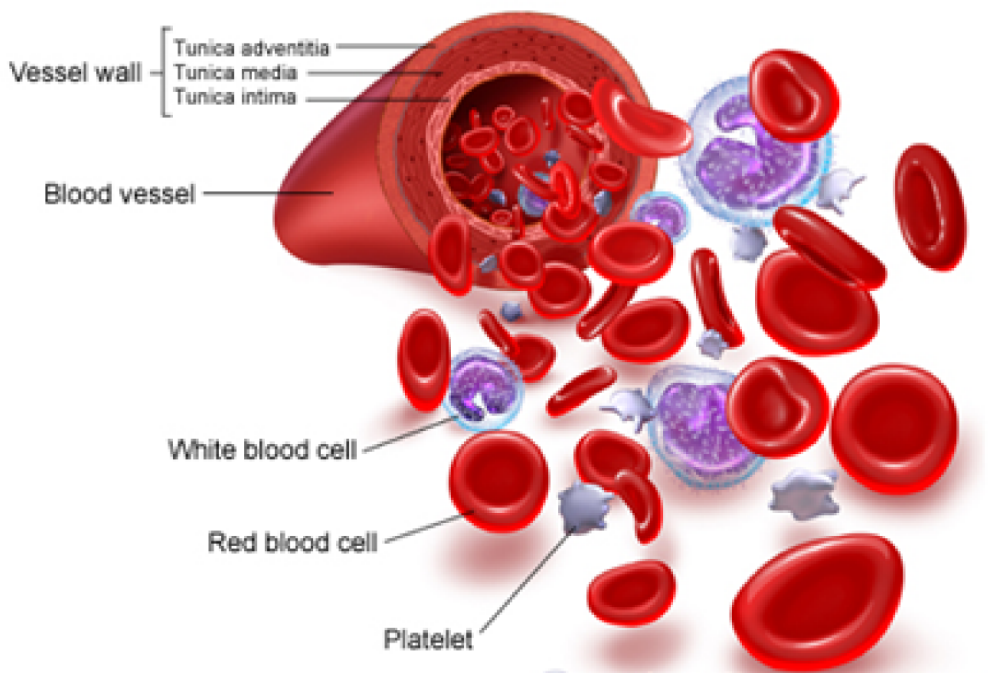

Figure 7.2: Schematic of a blood vessel and components of blood, from [110].

It is a non-Newtonian fluid whose flow properties play an essential role in many disease states including vascular disease, cancer and diabetes. Initial studies in the 1960s found that blood viscosity falls as shear rate increases [111]. While plasma by itself is only slightly more viscous than water, the remaining components of blood, RBCs deformability, aggregation, and relative volume of cells determine the actual flow properties of blood. Blood flows generally display a viscous as well as an elastic effect due to the flexible nature of its primary components. Therefore a viscosity for blood is defined only at shear rates above $100 \mathrm{~s}^{-1}$ where it is $\sim 5$ fold greater than water.

Blood plasma is a mixture of proteins, enzymes, nutrients, wastes, hormones and gases. WBCs are lined with microscopic projections called microvili, and are predominantly responsible for the immune response against foreign agents. Platelets are fragments of bone marrow cells that help prevent or stop bleeding, induce immune response when required, and cause blood clotting.

The relative volume of RBCs in blood, known as the hematocrit, is about $45 \%$ in healthy blood making RBCs the most important contributing factor to flow properties of 
blood. RBCs transport oxygen from the lungs to other tissues, and carry carbon dioxide from these tissues back to the lungs in the well studied mechanism of hemoglobin-oxygen binding. An RBC is an immotile flat discoid biconcave cell with no nucleus. The interior of the plasma membrane is lined with a 2-dimensional network of spectrin and actin conferring the cell its shape, resilience and flexibility. RBCs are able to deform when flowing through smaller blood vessels without increasing their surface area and then return to their original shape once a larger vessel is reached.

Many theoretical and experimental studies have been conducted to study the effects of mechanical properties of RBCs on blood flow [109; 108; 93; 111; 112]. Several disease states, including parasitic illness such as malaria, cause changes in erythrocyte shape and flexibility and can result in severe circulatory and vascular problems.

The Fähraeus-Lindquist effect refers to the formation of a reduced hematocrit plasma layer adjacent to the vessel wall. This effect is attributed to hydrodynamic lift of the deformable membrane vesicles (or cells) away from the high shear rate (and high shear stress) regions adjacent to the walls of the vessel resulting in a cell-free layer. An inertial drift force, perpendicular to flow lines, has been implicated in higher Reynolds number systems for spherical rigid particles [113; 114]. In Stokes flow, the same mechanism cannot account for this type of transverse (symmetry breaking) drift. In the case of deformable RBCs and membrane vesicles, tank treading motion in which the membrane rotates around the center of mass, allows the shape and orientation of the cell to remain constant with respect to the vessel wall, providing the necessary symmetry break for transverse motion with respect to flow lines.

In bounded shear flow, a drift away from walls of viscous origin has been reported for vesicles [115; 116; 117], and RBCs [92: 93]. Lift forces on RBCs have been estimated to range from 46 to $230 \mathrm{pN}$ [93].

Microfluidic devices have been instrumental in understanding the many complex aspects of blood flow including its dependence on shear rate, the importance of hematocrit on blood rheology, the origin of axial migration, and cell segregation.

\subsection{Experiment}

\subsubsection{Preparation of blood cells}

Blood was obtained from healthy cows and stored in EDTA to prevent coagulation and used within 2-3 days (generously provided by Melanie Scharfenstein from the Tierärztliches Institut, Göttingen). The RBCs were tested for their ability to maintain their shape and size in different suspension media (see Fig. 7.3). Before the experiment, the blood was washed twice with FCS and the whole blood hematocrit was preserved. We found this procedure not only allowed us to maintain the hematocrit but also that the RBCs maintained their biconcave shape (monitored visually) and that most (not all) adhesive molecules, including WBCs were removed and thus prevented clogging in the microflu- 
idic channels.
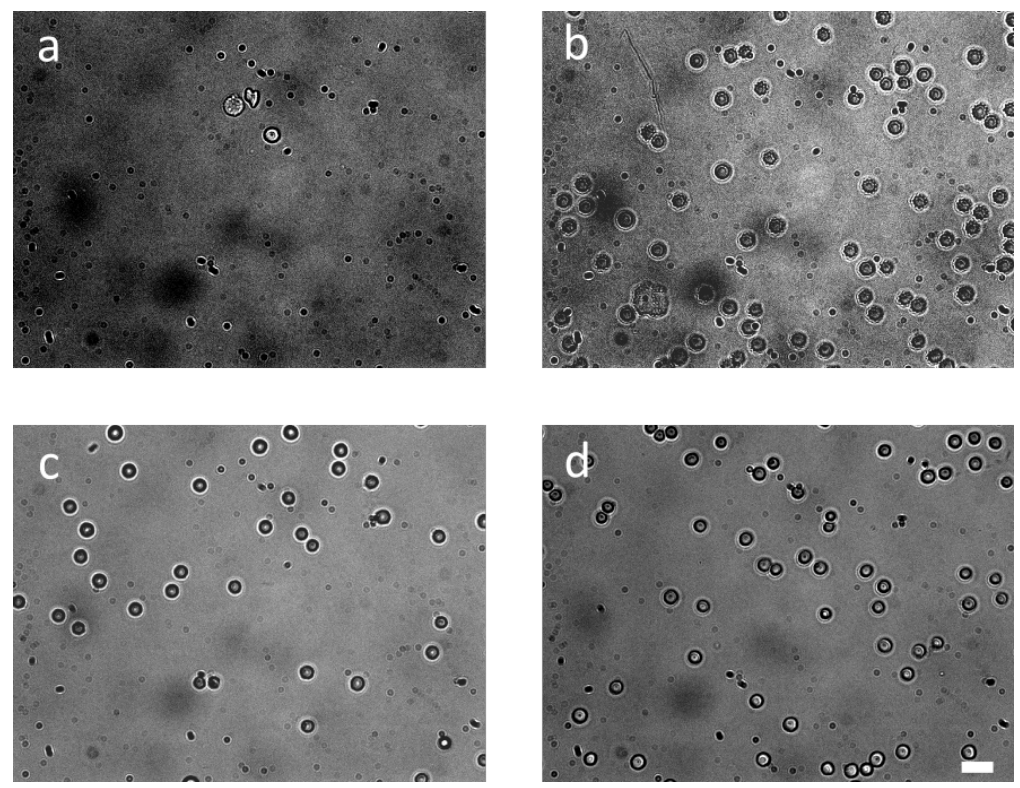

Figure 7.3: RBCs resuspended and diluted in (a) $0.9 \% \mathrm{NaCl}$ (15 min after resuspension), (b) whole blood (after 15 min at RT), (c) FCS (after 75 min at RT), and (d) TDB (cells appeared to burst open due to osmalarity after 15 minutes). Trypanosomes in FCS appeared healthiest for the longest period of time. Scale bar $15 \mu \mathrm{m}$

\subsubsection{Labeling and detection of trypanosomes}

During the experiment, the fresh blood preparation was mixed with trypanosomes. Due to the high concentration of RBCs in the microfluidic channel, detection of trypanosomes at the $\mathrm{z}=0$ focal plane was not possible using bright field transmission microscopy as we did in the previous experiments (see Chapter 6). Therefore, we sought to detect the trypanosome's location within the channel using fluorescent labeling.

Cells at $2 \times 10^{5}$ cells $/ \mathrm{ml}$ were washed twice and concentrated in TDB. They were then kept on ice with a 1:10 volume ratio of fluorescent dye to cell suspension for 10 minutes, followed by 3 more washing steps in TDB as reported previously [5]. Several dye concentrations were tested and optimized for signal brightness and cell viability. Both NHS Sulfo Rhodamine and NHS AMCA have been used to label the trypanosome cell surface. Since the flow velocities in our experiment were as high as $2 \mathrm{~mm} / \mathrm{s}$, exposure times on the recording camera (PCO) had to be kept as low as possible. A small exposure time in turn requires a relatively bright fluorescent signal for detection. High concentrations of the labeling dye were therefore necessary to detect the cell and to discern the cell shape. However, such high concentrations proved toxic to the cells as most cells appeared to be less motile even before injection into the channel. As mentioned in Chapter 1, the nucleus of the trypanosome is located approximately in the geometrical center of the cell. The 
nucleus is comprised primarily of DNA which can itself be labeled as shown in Fig. 7.4. Ethidium Bromide (excitation/emission), DAPI, and Hoechst 33342 were tested for cell toxicity, and fluorescent signal. Hoechst $33342\left(\lambda_{e x}=350 \mathrm{~nm}, \lambda_{e m}=461 \mathrm{~nm}\right)$ purchased from Thermo Scientifc (Germany) was selected as the optimal choice.

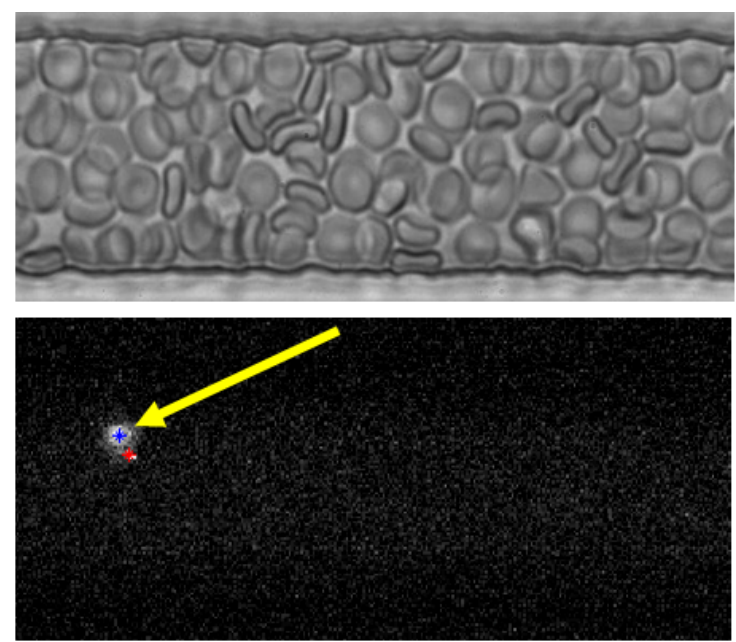

blue $=$ nucleus $\approx$ center of mass

red $=$ kinetoplast

Figure 7.4: Trypanosomes are not visible using bright field transmission illumination with such a high hematocrit. The nucleus and kinetoplast of Hoechst 33342 labeled cells (lower panel) allows for cell detection using image processing.

As seen in the Fig. 7.4 in addition to the nucleus, the kinetoplast, containing mitochondrial DNA is also labeled. The relative location of the kinetoplast to the nucleus which could also allow for a detection of an approximate cell orientation, given that the kinetoplast is always posterior to the nucleus. Labeled cells were mixed with the blood preparation and injected into the microfluidic channel. Note that while most the unbound dye is washed away, some remains in the cell suspension and when mixed with the blood preparation intercalates into the DNA of the remaining WBCs. RBCs do not contain DNA and therefore do not retain any of the dye. Using bright field illumination, the high concentration of RBCs complicate the measurement of flow velocities, therefore labeled WBCs prove useful in monitoring flow velocities under purely fluorescent illumination. It must be noted that cell labeling, no matter in what form, can be phototoxic upon exposure to light. However, in our setup, since the position of each cell is detected only once, phototoxic effects following illumination are not relevant.

The position of the trypanosome was automatically detected after the experiment using the image processing toolbox of Matlab. Briefly, each frame was converted to a black and white image using the im $2 \mathrm{bw}$ function of Matlab. The threshold value was adjusted to ensure that only the brightest spots would remain, thus ensuring that cells that were exclusively in the focal plane $(\mathrm{z}=0)$ were detected. Spurious pixels were eliminated us- 
ing the bwmorph(clean) filter. The position of the nucleus was determined based on the size of the bright spot, and then recorded relative to the channel wall. This automated routine allowed for the automatic detection of cell positions for as many movie frames as required and thus provide sufficient statistics to examine trypanosome position in blood flow.

Trypanosomes could not be immobilized in blood due to the presence of glucose in the suspension. Despite several washing steps, cells continued to be motile excluding the potential for comparison with immobilized cells.

\subsubsection{Cell free layer}

As discussed above, RBCs migrate away from walls resulting in a depletion layer at the walls of vessels. In experiments where blood samples were injected into straight channels of the same geometry $(23 \times 23 \mu \mathrm{m})$ as those studied in Chapter 6, this phenomenon was not observed to any obvious extent (as can been seen in Fig. 7.4). In a microfluidic setup, the cell free layer can be induced by introducing a constriction in the straight channel [118] as illustrated in Fig.7.5.

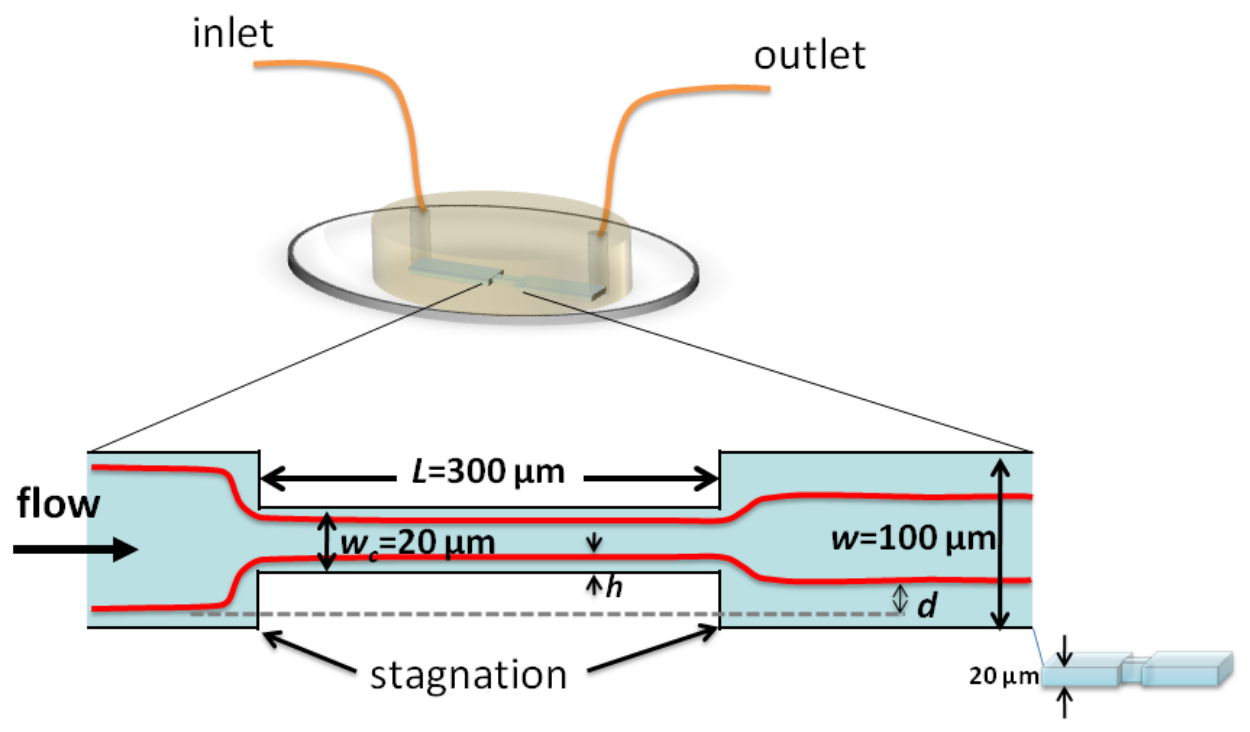

Figure 7.5: Introducing a constriction in a straight channel enhances the cell free layer thus mimicking the depletion near vessel walls observed in the circulatory network. The dimensions of the channel that were used (as shown) were selected to enhance the cell free layer. Red lines indicate the border of the cell free layer.

Within the constriction, while flow rate remains constant, the cross sectional area decreases resulting in an increase in velocity within the confinement, leading to a high shear rate. Upon the sudden return to the previous larger cross sectional area, the streamlines do not make a sharp turn towards the corners, but rather gradually move outwards in the expansion, and begin to fill the whole channel and then remain parallel to the walls. 
Thus, a small stagnation area is seen at the corners (indicated in Fig. 7.5) before and also after the constriction.

Finite element method (FEM) simulations Fig. 7.6) were conducted using COMSOL Multiphysics and show the effect of such a geometry on the velocity and streamline profile.

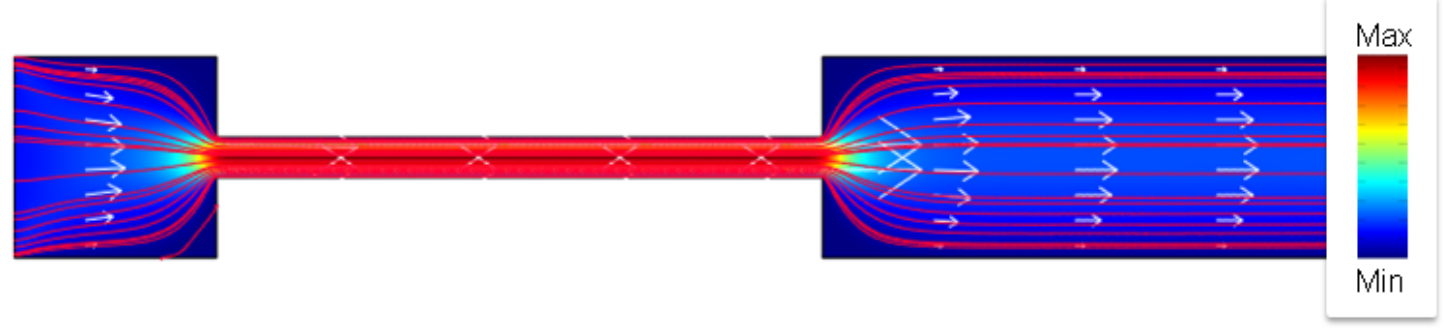

Figure 7.6: FEM simulation to test the effect of a constriction on the streamline profile. Surface and arrow plot show relative flow velocities; streamlines are in red.

Previous work by Faivre [119] showed that the strongest depletion layer was obtained with increases in the length of the constriction, the concentration of cells, the viscosity of the suspending media, the cell deformability, and the volume of the cells and a decrease in the width of the constriction [119]. The highest hematocrit used by Faivre was $2.6 \%$ [119]. However, for our experiments, we chose physiological hematocrit levels of whole blood levels of about $45 \% \mathrm{v} / \mathrm{v}$. A freshly prepared blood sample flowing through such a channel, shown in Fig. 7.7. clearly indicates that despite such a high hematocrit level, a depletion layer can be induced near the walls. Detection of fluorescently labeled cells is optimal at the center plane in a channel that is $20 \mu \mathrm{m}$ or less in height due to density of the RBCs in our experiment. We therefore chose a channel that was $20 \mu \mathrm{m}$ high, and $100 \mu \mathrm{m}$ wide. The confinement was with a high length to width ratio; $300 \mu \mathrm{m}$ long and $20 \mu \mathrm{m}$ wide as in Fig.7.5.

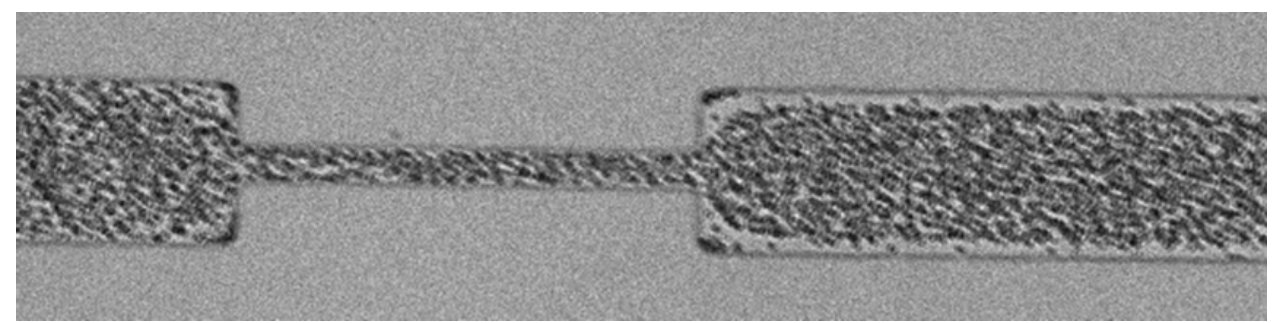

Figure 7.7: Cell free layer of $\sim 10 \mu \mathrm{m}$ shown in a channel of dimensions shown in Fig. 7.5. Stagnation point are seen at the corners immediately before and after the constriction. The cell free layer does not persist as far downstream as reported by Faivre M [119].

Faivre $\mathrm{M}$ et al. reported that the depletion layer is maintained well beyond the constriction. However, we found that cells begin to fill the depletion layer $\sim 1 \mathrm{~mm}$ past the 
constriction. This difference could be due to the much greater hematocrit in our setup - RBC collisions are more significant, and may overcome any drift toward the channel center, causing them disperse outward much faster. Therefore, trypanosome center of mass position was recorded immediately after the constriction and about $2 \mathrm{~mm}$ further downstream of the constriction. At this distance the depletion layer was significantly diminished.

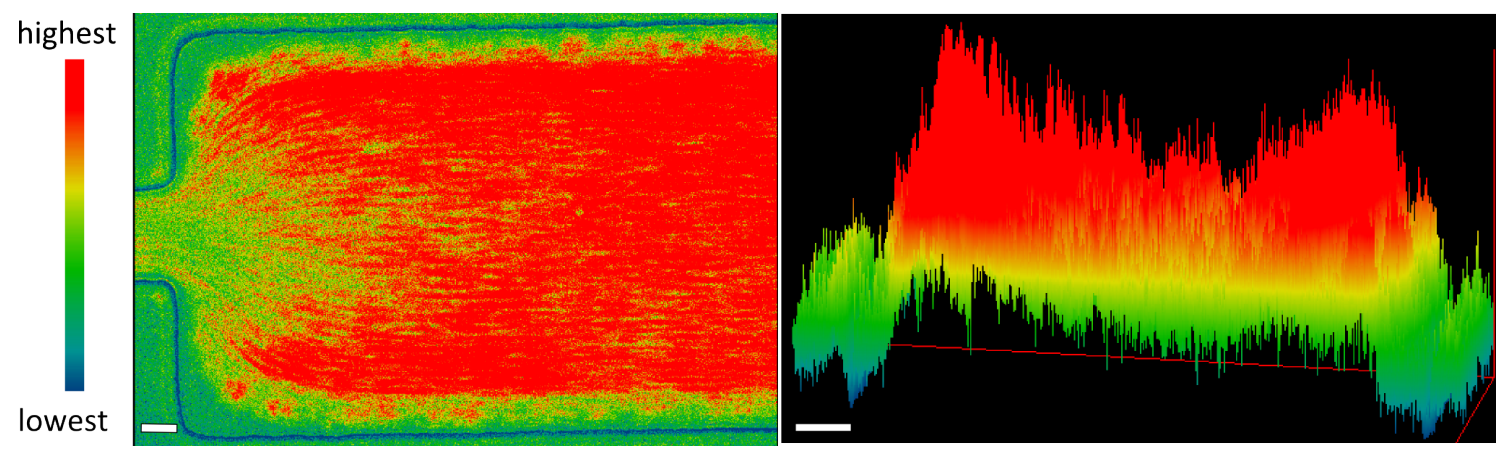

Figure 7.8: Color surface plot of RBC density visualized by the relative grey scale value of a processed average image. The streamlines followed by the RBCs are clearly seen. The cross section on the right shows that the RBC density is highest at the two ends closest to the channel walls. Scale bar $10 \mu \mathrm{m}$.

Due to the high density of red blood cells within the channel, ascertaining the exact flow profile within the channel is not trivial. We exploit the contrast of the recordings in the movies of the flowing blood cells to obtain a qualitative measure of the width of the cell free layer. The average of at least 200 processed frames of a movie of RBCs flowing out of a constriction yields the relative gray scale values shown in Fig. 7.8. We thus find that the cell free layer is $\sim 15 \mu \mathrm{m}$ in width. The streamlines followed by the RBCs are clearly seen. After the constriction we see that the highest number of RBCs are found in the streamlines toward the edges of the cell free layer (Fig. 7.8). Cells closest to the wall migrate across streamlines when passing through the constriction, thus showing the maximum cell density near the end of the cell free layer downstream of the constriction. This observation was also made in [119].

\subsection{Results}

Fig. 7.9 a shows the center of mass distribution of swimming trypanosomes in blood at various flow velocities in a straight symmetric channel $(23 \times 23 \mu \mathrm{m})$. The distribution looks very similar to distributions in Fig. 6.12 for experiments without blood.

Except at $0.1 \mathrm{~mm} / \mathrm{s}$ where self propulsion dominates, the distribution is strongly peaked at the center of the channel for all velocities ranging from $0.4 \mathrm{~mm} / \mathrm{s}$ to $1.0 \mathrm{~mm} / \mathrm{s}$, with a steep increase after $\sim 5 \mu \mathrm{m}$. The trend also remains the same as in Fig. 6.12; as 
a

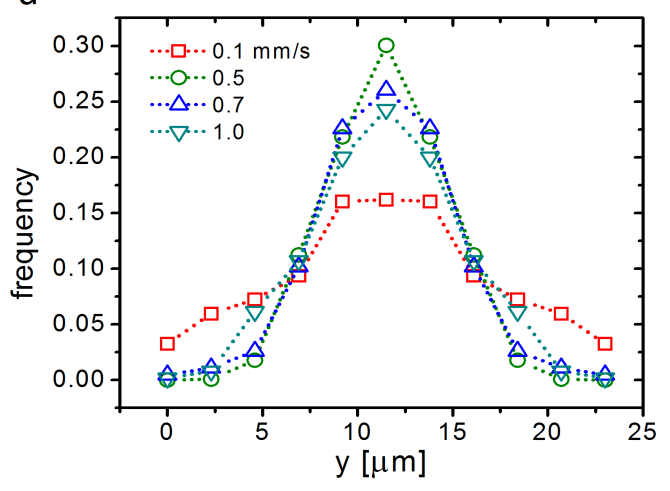

b

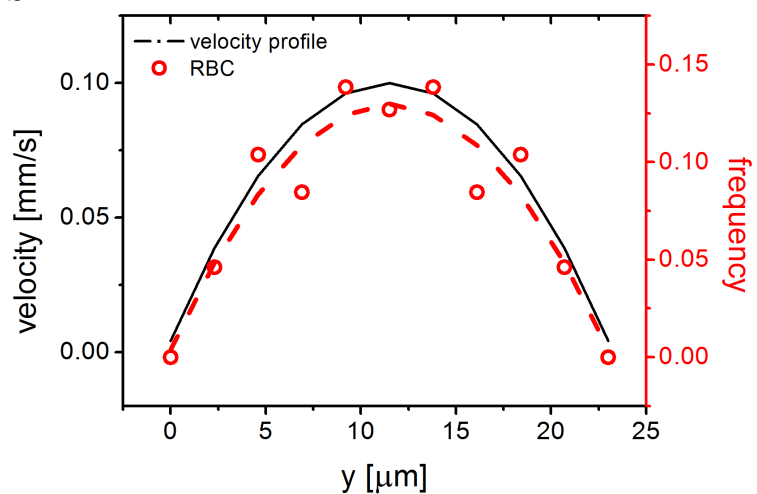

Figure 7.9: a. Trypanosomes distribution in blood at various velocities. b. Distribution of RBCs in the same channel; red dotted line is an aid to the the eye. In black the velocity profile is shown for the same channel calculated from Eq. 6.2.

the velocity is increased, the peak of the distribution is reduced further. The shear induced migration discussed above appears to continue to play the same role to push most cells toward the center. To construct the distribution of RBCs, shown in Fig. 7.9p, flowing through the channel, the number of cells that flowed through a specified point were counted manually in $2 \mu \mathrm{m}$ intervals and were then normalized by the total number of cells. The distribution of RBCs reflects the parabolic flow profile shown in the same figure.

Two observations can be made from Fig. 7.9 (1) the axial migration of RBCs toward the center of the channel cannot be reproduced in this setup with such a high hematocrit, and (2) the center of mass distribution of trypanosomes does not reveal a tendency to swim close to the channel walls at any of the tested flow velocities.

As described in the experiment section above we found that a constriction in the channel could be utilized to induce a depletion layer of RBCs at the channel walls. Rather than the parabolic profile we observed in the straight channels, the velocity profile is no longer symmetric in the $\mathrm{z}$-axes due to the low aspect ratio of the channel geometry (see Fig. 7.5).

To understand the effect of the constriction on trypanosomes, we first flowed a dilute suspension of trypanosomes through the constriction in culture medium (i.e without blood) at a low flow velocity of $0.1 \mathrm{~mm} / \mathrm{s}$ and high flow velocity of $2 \mathrm{~mm} / \mathrm{s}$. We examined the lateral center of mass distribution in the channel immediately after the constriction (Fig. 7.10) and $\sim 2 \mathrm{~mm}$ downstream (Fig. 7.11) of the constriction. Effects of self propulsion and size, shape and density of the cell could also be discerned from the differences in behaviour of live and immobilized cells (see Chapter 6 for details on cell immobilization).

Fig. 7.10 shows the distribution of cells recorded immediately after the constriction 


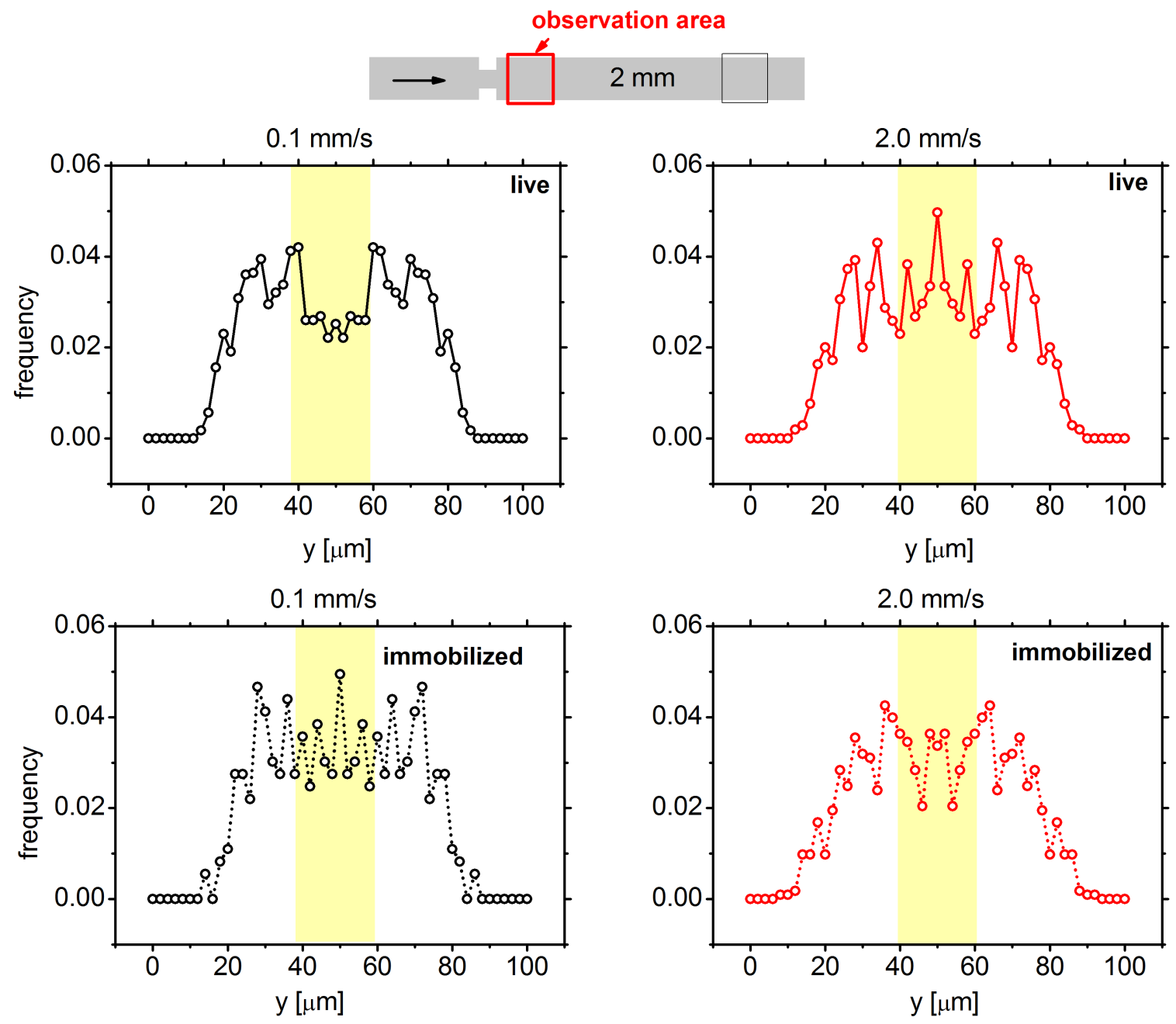

Figure 7.10: Distribution of center of mass of live (top panels) and immobilized (lower panels) cells at $0.1 \mathrm{~mm} / \mathrm{s}$ (black) and $2.0 \mathrm{~mm} / \mathrm{s}$ (red) immediately after the constriction (as illustrated by the schematic). Yellow shaded area corresponds to the width and position of the constriction.

at $0.1 \mathrm{~mm} / \mathrm{s}$ and $2 \mathrm{~mm} / \mathrm{s}$ for both live and immobilized cells. We see that that due to high shear at the walls in the constriction, most cells come to the center of the channel and seem to follow the stream lines seen in Fig. 7.6 after exiting the constriction and do not repopulate the area closest to the walls. Thus, the distributions show a complete absence of cells for a distance of up to $\sim 20 \mu \mathrm{m}$ away from the walls. A fairly rapid increase starting at this $20 \mu \mathrm{m}$ point is then followed by a relatively uniform probability of finding a trypanosome out of the depletion layer. As shown in Chapter 6 at flow velocities of $0.1 \mathrm{~mm} / \mathrm{s}$ self propulsion seems to play a significant role in cell positioning in flow. We see again in Fig. 7.10 that only at $0.1 \mathrm{~mm} / \mathrm{s}$ we see a decrease in live cells at the location corresponding to the constriction - likely due to self propulsion. In contrast, in the straight channel, with a parabolic flow profile, the distribution in Fig. 7.9 indicated 
that cells are most likely to be found at the center of channel. The constriction followed by the sudden expansion causes the cells to be focused in the center of the channel, and away from the walls.
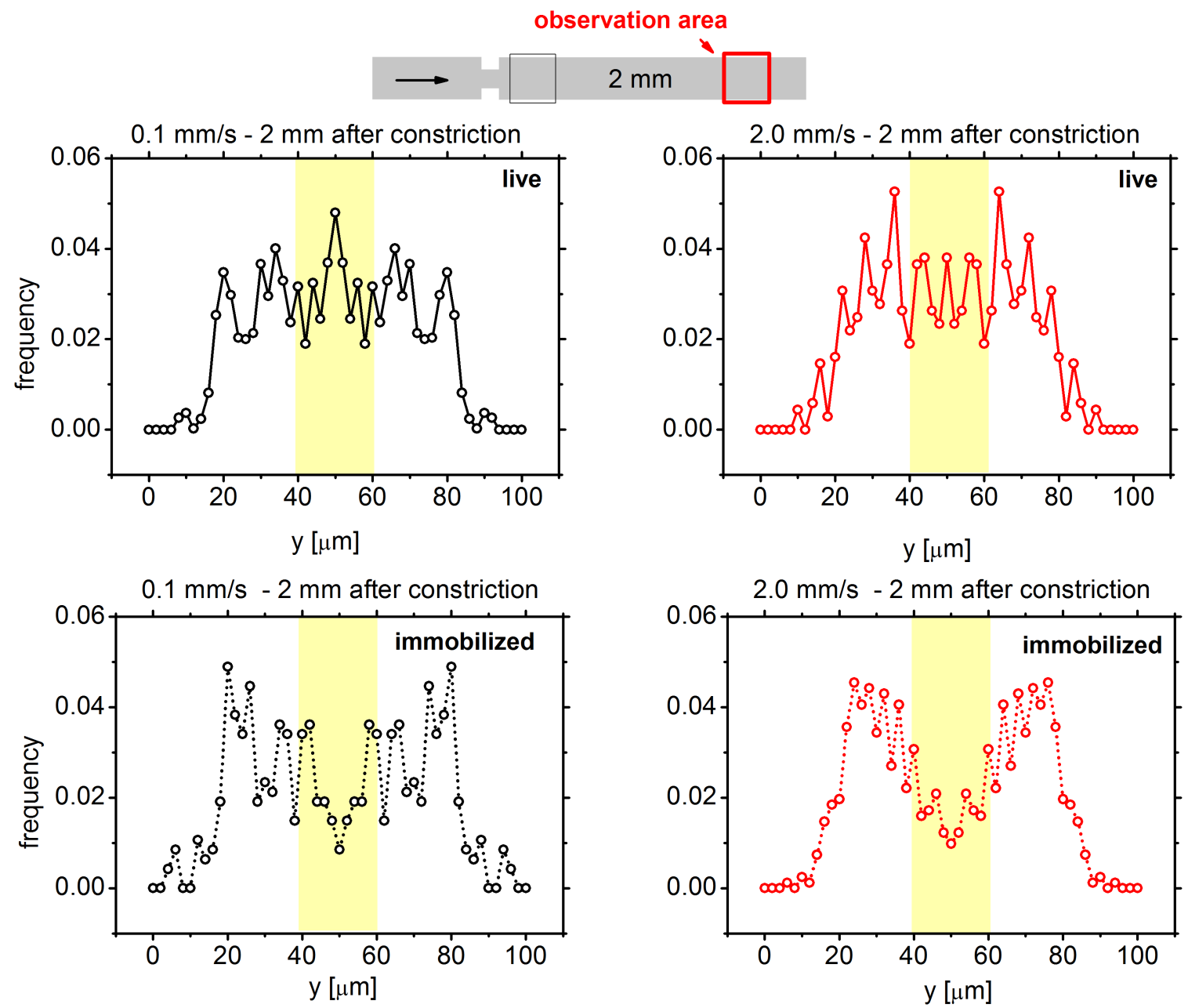

Figure 7.11: Distribution of center of mass of live (top panels) and immobilized (lower panels) cells at $0.1 \mathrm{~mm} / \mathrm{s}$ (black) and $2.0 \mathrm{~mm} / \mathrm{s}$ (red) $\sim 2 \mathrm{~mm}$ after the constriction (as illustrated by the schematic). Yellow shaded area corresponds to the width and position of the constriction.

Approximately $2 \mathrm{~mm}$ further down the constriction the distribution of trypanosomes (Fig. 7.11) is not dramatically different near the walls - we still observe a depletion layer of about the same width. However, immobilized cells are predominantly found halfway from the channel center to the channel wall at both $0.1 \mathrm{~mm} / \mathrm{s}$ and $2 \mathrm{~mm} / \mathrm{s}$ flows. Thus a there is a strong depletion both near the walls and at the center of the channel, corresponding the location of the constriction (shaded area in Fig. 7.11). At $2 \mathrm{~mm} / \mathrm{s}$ the distribution of live cells also shows an indication of depletion at the center of the channel similar to the immobilized cells but live cells, at $0.1 \mathrm{~mm} / \mathrm{s}$ do not show a drop in the distribution at the channel center. While cells may be migrating toward channel walls 
due to diffusion, a lift force may be counterbalancing this effect resulting in the drop in the center of distribution observed in Fig. 7.11

Importantly, we note that the depletion layer near the walls has not changed $2 \mathrm{~mm}$ downstream of the constriction and that it remains constant at both flow velocities. These results point to a lift force that, like in the case of RBCs, pushes the trypanosomes toward the center of the channel.

Given the complexity of the trypanosome body and the geometry of our system, a quantitative description is difficult to achieve. However, Faivre et al. [118] used the functional form of the idealized theory suggested by Olla [120] to derive an expression for the drift induced by the constriction on an ellipsoid cell:

$$
d \approx 6 \kappa \frac{L R^{3} w_{c}}{w^{2} h^{2}}
$$

where $L$ is the length of the constriction, $w$ and $w_{c}$ are the widths of the main channel and the constriction respectively, $R$ is the radius of the sphere of the same volume as the cell, and $h$ is the distance of the particle to the wall in the constriction, as illustrated in Fig. 7.5. Here $\kappa$ is a dimensionless parameter dependent on the orientation of the cell and its shape (eccentricity) which in turn are dependent on the viscosity ratio inside the cell and the suspending medium $\eta_{i} / \eta_{o}$. Using the experimental measurements, assuming $R=$ $5 \mu \mathrm{m}$, we estimate $\kappa_{\text {tryp }}=0.007$ from Eq. 7.1 - intriguingly this is in close agreement with the experimental value found for RBCs of 0.01 [119]. Indeed Faivre found experimentally that $d$ was a linearly proportional to $L w_{c} / w^{2}$ [119] with

$$
d=0.26+0.24 \frac{L w_{c}}{w^{2}} .
$$

Given the dimensions of the channel utilized in this study, we would therefore expect $d=18.26 \mu \mathrm{m}$ - in excellent agreement with Fig. 7.10 and Fig. 7.11 which show enhancement in the cell free layer of $\sim 17 \mu \mathrm{m}$ after the constriction. Together these observations indicate that the lift force experienced by trypanosomes is likely to be of the same order as RBCs.

We now examine how these observations are affected when trypanosomes swimming in blood are forced through the constriction. Fig. 7.12 shows the distribution of live trypanosomes at $0.1,0.4$ and $2.0 \mathrm{~mm} / \mathrm{s}$ immediately after the constriction, and $\sim 2 \mathrm{~mm}$ after the constriction in $2 \mu \mathrm{m}$ intervals. The RBC profile derived from the same procedure as in Fig. 7.8 is also shown as a dashed black line. An overall structure in the distribution of trypanosomes and RBCs is apparent.

Immediately after the constriction, (Fig. 7.12a) shows that there is a peak in the distribution of trypanosomes about $30 \mu \mathrm{m}$ away from the channel wall. Further downstream (Fig. $7.12 \mathrm{~b}$ ), we find that these peaks have shifted even further away from the center and are now within a cell length distance $(\sim 17 \mu \mathrm{m})$ away from the channel walls as seen in Fig. $7.12 \mathrm{p}$. This peak in the trypanosome distribution becomes more pronounced as the 


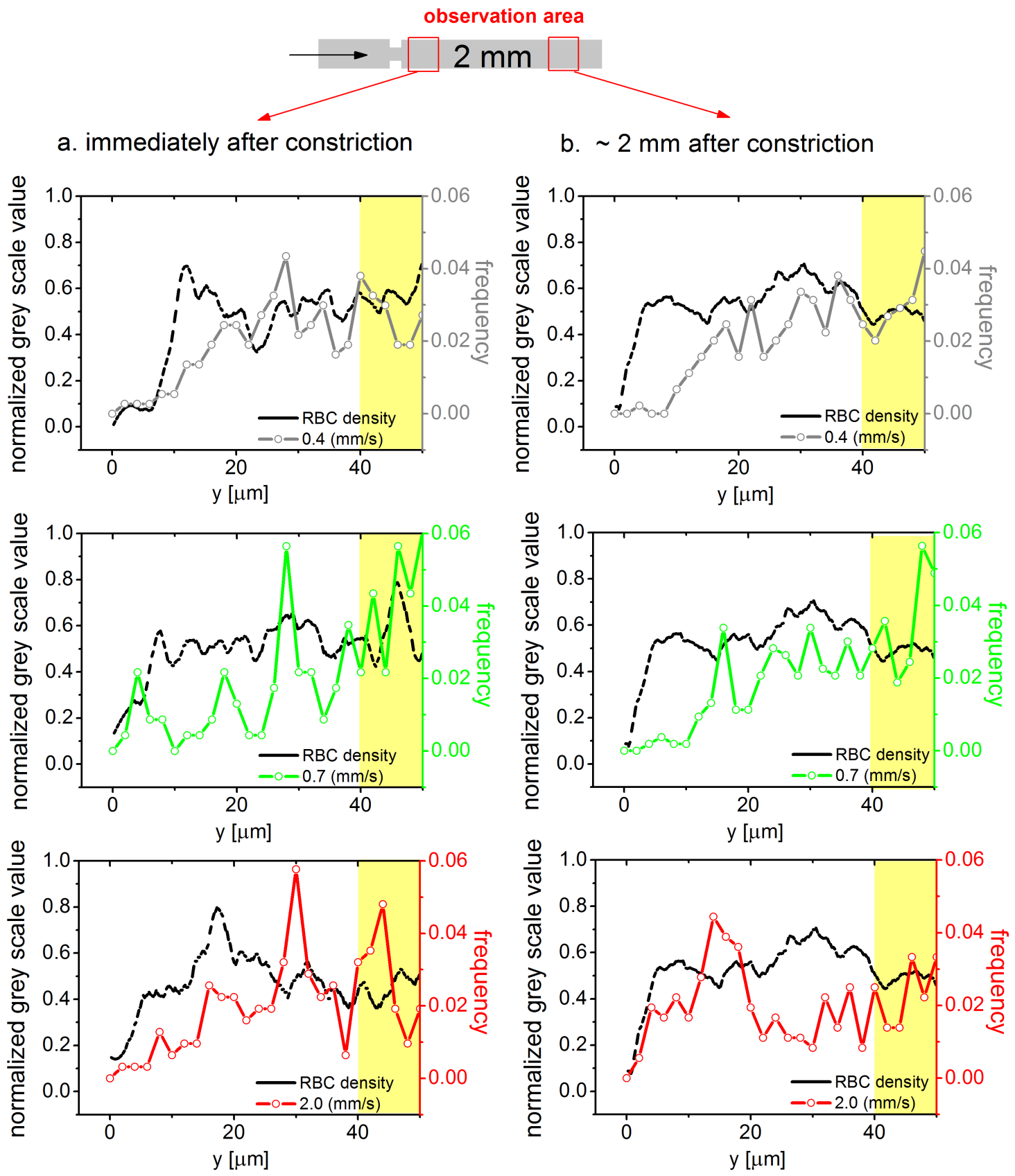

Figure 7.12: Trypanosomes distribution in blood flow (a) immediately after a constriction and (b) $\sim 2 \mathrm{~mm}$ after the constriction at $0.1,0.7$ and $2.0 \mathrm{~mm} / \mathrm{s}$ average flow velocities. Only half-width of the channel is shown. Black dashed lines show the grey scale values corresponding to the RBC density. Yellow shaded area corresponds to the relative position of the constriction.

velocity is increased, but remains in the same lateral position both immediately after the constriction and further downstream.

The distributions of the RBCs (based on averaged grey scale values) are quite distinct from that of the trypanosomes at both locations downstream of the constriction. 
Immediately after the constriction, trypanosome density appears to reduce more gradually towards the walls as compared to the RBCs which seem to exhibit a much more dramatic drop in density closer to the walls.

Interestingly, the shift in peak trypanosome distribution is reminiscent of white blood cell dynamics in blood flow. RBCs are known to aggregate, forming a coherent rouleau, and exclude WBCs from the bulk solution resulting in WBC margination [121; 122]. The effect is enhanced due to the difference in cell stiffness - WBCs are gradually displaced by the deformable particles (RBCs). Further, expansions in microfluidic devices such as the one in our system, have be shown to contribute to reorganize in response to change in geometry [123] contributing greatly to WBCs migration toward boundaries. A WBC that has established contact with a vessel wall is further subjected to bouncing forces from passing RBCs, promoting adhesion to the walls. It is likely that the same mechanisms acting on WBCs in blood flow promote trypanosome margination to vessel walls, since as discussed in Chapter 6, trypanosomes do seem to be stiffer than RBCs

In our experiments, we $d_{R B C} \approx 10 \mu \mathrm{m}$ immediately after the constriction - much lower than what is expected from Eq.7.2 indicating that collisions between RBCs due to the hematocrit in our system are significant. It is likely that hematocrit and vessel network geometry (constriction/expansion) play a critical role in axial migration and pathogen penetration through the epithelium and therefore require further attention in order to address physiologically relevant questions.

\subsection{Potential as a diagnostic tool}
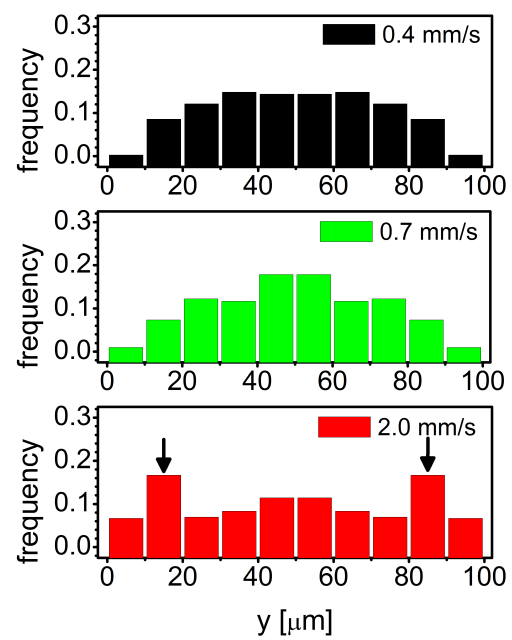

Figure 7.13: Distribution of trypanosomes (constructed using $10 \mu \mathrm{m}$ bins) suspended in blood $2 \mathrm{~mm}$ downstream from the constriction shows trypanosome enrichment closer to the edges (black arrows).

An interesting consequence of this study is the ability to enrich trypanosome con- 
centration close to the walls of the channel. This is well illustrated in the reconstructed distribution of trypanosomes (using a larger bin size) in blood flow $2 \mathrm{~mm}$ downstream of the constriction in Fig. 7.13 .

As mentioned in Chapter 1, the most commonly used diagnostic tool for trypanosomiasis is a blood smear. Depending on the stage of infection parasitemia (proportion of trypanosomes in blood volume) can be very low - impeding proper diagnosis. Several separation techniques have been suggested to pre-concentrate a blood sample with the pathogenic parasite before microscopic examination.

Microfluidics has been widely suggested for medical applications such as diagnostics. Deterministic lateral displacement (DLD) is one such tool based on the interaction of particles under low Reynolds number conditions with an ordered array of obstacles under an imposed flow. The spacing between the micrometer sized obstacles is of critical radii and only particles smaller than a critical size can flow through the gap, while larger particles are forced to change streams through interaction with the posts. Holm et al. recently showed that trypanosomes could be separated from blood in microfluidics by utilizing DLD with higher sensitivity that older techniques [124]. However, precise spacing of the obstacles is required and clogging by larger particles within narrower streams can reduce the effectiveness of such a device.

The simple microfluidic design presented above with a long constriction allows for the enrichment of trypanosome within a blood sample thus increasing the sensitivity of downstream diagnostics test such as blood smears.

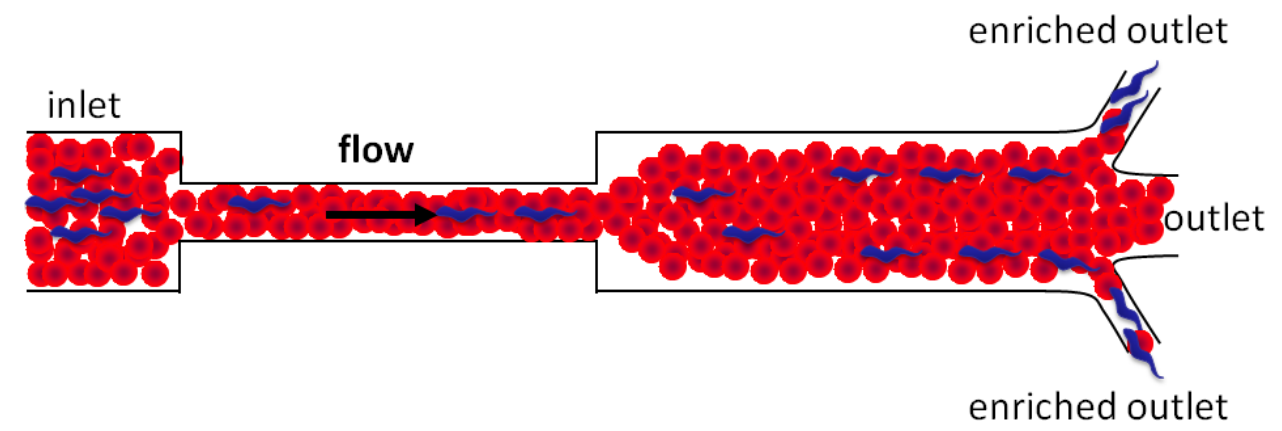

Figure 7.14: Trypanosome enrichment in blood sample through margination.

In Fig. 7.14 we illustrate a channel design that exploits trypanosome margination to enrich the concentration of trypanosomes in a blood sample. Such an enrichment would facilitate detection of trypanosomes in blood samples requiring diagnosis for trypanosomiasis. It also likely that such a device could be used not only for the detection of trypanosomes, but also for similar species of extracellular parasites such as Leishmania (causative agents of Leishmaniasis).

The process of margination has been attributed to cell stiffness through experiments with rigid and healthy flexible RBCs. Malaria infected RBCs are stiffer due to a cross 
linking of the outer spectrin network of the cell [125]. Hou et al. showed that through a similar narrowing within a long straight channel, healthy RBCs displacing rigid ones; the resulting cell margination could be used to separate the two species of RBCs [125].

\subsection{Summary and outlook}

In this chapter, the presence of a simple constriction allowed us to recreate a blood vessel environment with a cell free layer near boundaries. In dilute suspension of trypanosomes the cell free layer persisted even $2 \mathrm{~mm}$ downstream of the constriction pointing to a lift force causing cells to be pushed away from walls.

In blood flow, we found that downstream of the confinement, trypanosome density peaks within a distance less than cell length away from the channel walls. Interaction with adhesive factors at the vessel walls may reduce the effect of any shear force causing the cell to move away from the wall, allowing the trypanosome to be caught at the surface through cytoadhesion and penetrate through the tissue.

We plan to use microfluidic design to study examine trypanosome's ability to cross through tightly-knit barriers in blood flow. Barriers are constructed in the form of spacer pillars which may in turn be coated with ECM-like material such as collagen. Such coatings have been achieved in the past with ECM components [126].

Indeed first experiments (Fig. 7.15) have shown that trypanosomes can make their way through narrow gaps even in the presence of flow. Further detailed experiments are required to optimize physical conditions such as flow velocity, gap widths, coating densities etc for these studies.

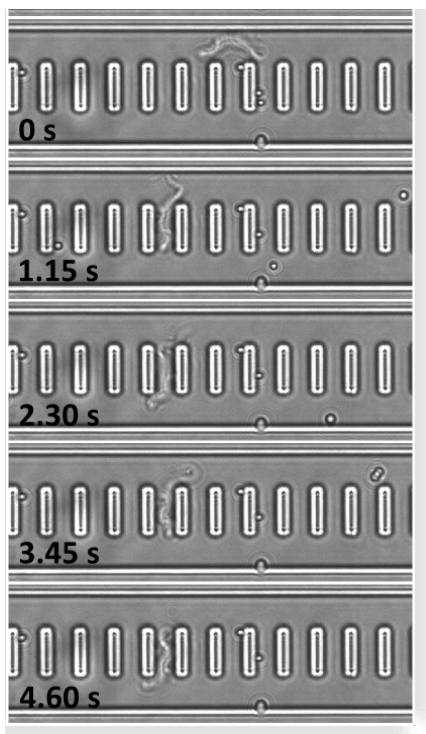

Figure 7.15: Trypanosomes can make their way through narrow gaps even in flow. Here spacers are $3 \mu \mathrm{m}$ apart, flow is $\sim 0.1 \mathrm{~mm} / \mathrm{s}$. 


\section{Conclusions}

Applications of cell motility studies are diverse and far reaching, from the prevention of infection in catheterized patients to the design of artificial microswimmers. Trypanosomes, unicellular parasitic pathogens, swim by a mechanism that remains to be fully elucidated. They represent a unique model organism for several reasons. Firstly, they swim with the aid of a flagellum that is, unlike other well known flagellates, attached to the length of their body, causing changes in whole cell body shape during swimming. Secondly, blood stream form trypanosomes have adapted to the high shear stress flow of the host's circulation. Finally, the hydrodynamic drag created on the surface of the cell membrane during swimming is a means to sweep away host antibodies from the cell surface, allowing the trypanosome to remain undetected by the immune system of the host. This work therefore addresses the need for a detailed, quantitative perspective of trypanosome motility.

In a nutrient rich homogeneous environment, trypanosomes swim in one of three distinct motility modes: random walk, directional persistence, and an intermediate mode in which they exhibit a combination of both. The experimentally observed motility modes correspond to distinct physical movements and can be attributed to distinct cell shape conferred mainly by flagellum dynamics. We found that directional persistence arises only with stretched cells implying there are significant stiffness differences within a single population. Further, initiation of cell division, beginning with emergence of a second flagellum at the base, correlates to directional persistence. Cells that swim faster also exhibited strong fluctuations in velocity. Statistical analysis allowed us to develop a model based on Langevin equations to show that the rapid movements of the body (with time scales in the order of $0.1 \mathrm{~s}$ ) are a result of an active process. Using high-speed microscopy with a frame rate of $1000 \mathrm{~Hz}$, the small time scales obtained from the model were confirmed. Moreover we found that cell swimming is driven by microscopic movements of the cell ends that are $\sim 50$ fold faster than the whole cell swimming speed.

Trypanosome motility in environments of tunable mesh size and viscosity were also studied. A decrease in the overall speed of trypanosomes in collagen and actin networks was observed. However, scaling analysis indicated that some persistent cells become even more directional - likely due to confinement arising from the entangled network fibers. Using dextran as a viscosity tuning agent, we found that trypanosomes are able 
to swim through high viscosities previously shown to immobilize other microorganisms. Increases in viscosity resulted in uncorrelated behaviour, as shown in turn angle distributions, due to a rocking motion of the cell. We further demonstrated first results toward using the movement of self propelling microorganisms such as trypanosomes as active probes to estimate the mesh size and measure the elastic modulus of surrounding polymer networks.

When in containers, many microswimmers are attracted by boundary walls due to hydrodynamic interactions. Once a flow is introduced, diverse behaviours have been observed, including upstream swimming. In microfluidic channels, most active cells were found near walls in the absence of any flow. In pressure driven flows ranging from 0.1 $\mathrm{mm} / \mathrm{s}$ to $1.0 \mathrm{~mm} / \mathrm{s}$, trypanosomes are lifted away from the channel walls to the center. In flows of less than $0.1 \mathrm{~mm} / \mathrm{s}$ they are highly aligned with boundary walls and swim upstream in sine wave-like trajectories. As flow velocities increase even further, cells tumble (by rotating) and their behaviour converges with that immobilized cells.

Finally, microfluidics was used as a tool to mimic blood flow. A simple constriction followed by an expansion acted as a sorter which allowed for cell separation pushing most trypanosomes closer to channel walls, while red blood cells remained in the center of the channel. The effect of cell separation was enhanced in the channel further downstream of the constriction. We could estimate the lift forces acting on trypanosomes to be of the same order of magnitude as red blood cells. However, when red blood cells and trypanosomes are mixed together, stiffer trypanosomes migrate out towards the boundary walls. Thus the circulatory system, comprised of a network of interconnected narrowing and widening vessels is likely to enhance the parasite's migration to vessel walls and may promote penetration through the epithelium, including the blood brain barrier. We suggest that this mechanism could be exploited for trypanosome enrichment for diagnostic purposes.

We anticipate that biophysical studies of other microorganisms may benefit from many of the analysis tools presented here. In particular, tracking the average end to end distance allows for a relative measure of stiffness for elongated cells by drawing an analogy to a worm like chain. Varied elastic environments are found in nature. We foresee that the elastic characteristic of these environments may be extracted from the trajectories of their micro-inhabitants. Further, microfluidic channels may be utilized to impose a flow load on flexible cells, and to extract stiffness properties from the consequent cell stretching or unfolding.

The results from this thesis improve our understanding of trypanosome locomotion in minimal and biomimetic environments which may in turn provide a basis for further studies in diagnostic and treatment options for trypanosomiasis. The work provides insight into low Reynolds number swimmers, and in particular the swimming mechanism of this unique creature. 


\section{List of Abbreviations}

$\begin{array}{ll}\text { ADP } & \text { Adenosine diphosphate } \\ \text { ATP } & \text { Adenosine triphosphate } \\ \text { BBB } & \text { Blood Brain Barrier } \\ \text { BSF } & \text { Blood Stream Form } \\ \text { CNS } & \text { Central Nervous System } \\ \text { DNA } & \text { Deoxyribonucleic Acid } \\ \text { ECM } & \text { Extracellular Matrix } \\ \text { FAZ } & \text { Flagellum Attachment Zone } \\ \text { FCS } & \text { Fetal Calf Serum } \\ \text { FEM } & \text { Finite Element Method } \\ \text { HMI9 } & \text { Trypanosome Culture Medium (formulated by Hirumi \& Hirumi) } \\ \text { IW } & \text { Intermediate Walker } \\ \text { MSD } & \text { Mean Squared Displacement } \\ \text { MT } & \text { Microtubule } \\ \text { PF } & \text { Procyclic Form } \\ \text { PFR } & \text { Paraflagellar Rod } \\ \text { PW } & \text { Persistent Walker } \\ \text { RBC } & \text { Red Blood Cell } \\ \text { RNA } & \text { Ribonucleic Acid } \\ \text { RW } & \text { Random Walker } \\ \text { TDB } & \text { Trypanosome Dilution Buffer } \\ \text { VSG } & \text { Variable Surface Glycoprotein } \\ \text { WBC } & \text { White Blood Cell }\end{array}$





\section{Bibliography}

[1] "jeffwyner - VSFX studio II - microbe scene." http:/ / theboxthebox.com/studio2/microbes.html.

[2] "MinnPost - comments: Eric black ink." http://www.minnpost.com/ericblack/comments_eric_black_ink/?blog_post_id=723.

[3] "Lyme green australia: Spiroplasma." http://lymegreenaustralia.blogspot.com/2009/09/spiroplasma-creutzfeldt-jakob-disease.html.

[4] R. Dreyfus, J. Baudry, M. L. Roper, M. Fermigier, H. A. Stone, and J. Bibette, "Microscopic artificial swimmers," Nature, vol. 437, pp. 862-865, Oct. 2005.

[5] M. Engstler, T. Pfohl, S. Herminghaus, M. Boshart, G. Wiegertjes, N. Heddergott, and P. Overath, "Hydrodynamic Flow-Mediated protein sorting on the cell surface of trypanosomes," Cell, vol. 131, pp. 505-515, Nov. 2007.

[6] R. Broadhead, H. R. Dawe, H. Farr, S. Griffiths, S. R. Hart, N. Portman, M. K. Shaw, M. L. Ginger, S. J. Gaskell, P. G. McKean, and K. Gull, "Flagellar motility is required for the viability of the bloodstream trypanosome," Nature, vol. 440, Mar. 2006.

[7] P. P. Simarro, J. Jannin, and P. Cattand, "Eliminating human African trypanosomiasis: Where do we stand and what comes next?," PLoS Med, vol. 5, p. e55, Feb. 2008.

[8] "WHO diseases covered by NTD department." http://www.who.int/neglected_diseases/diseases/en/, 2010.

[9] P. Caramello, “Search." http://www.cdfound.to.it/_search.htm, 2010.

[10] "WHO human African trypanosomiasis." http://www.who.int/trypanosomiasisafrican/en/, 2010.

[11] D. Barry, Trypanosomes: After The Genome. Routledge, 1 ed., Feb. 2007.

[12] K. S. Ralston, A. G. Lerner, D. R. Diener, and K. L. Hill, "Flagellar motility contributes to cytokinesis in Trypanosoma brucei and is modulated by an evolutionarily conserved dynein regulatory system," Eukaryotic Cell, vol. 5, pp. 696-711, Apr. 2006.

[13] D. Maric, C. L. Epting, and D. M. Engman, "Composition and sensory function of the trypanosome flagellar membrane," Current Opinion in Microbiology, vol. 13, Aug. 2010.

[14] M. Oberholzer, M. A. Lopez, B. T. McLelland, and K. L. Hill, "Social motility in African trypanosomes," PLoS Pathogens, vol. 6, no. 1, 2010. 
[15] E. Purcell, "Life at low reynolds number," American Journal of Physics, vol. 45, pp. 311, 1977.

[16] N. Cohen and J. Boyle, "Swimming at low Reynolds number: a beginners guide to undulatory locomotion," Contemporary Physics, vol. 51, pp. 103-123, Mar. 2010.

[17] H. Wada and R. R. Netz, "Hydrodynamics of helical-shaped bacterial motility," Physical Review E, vol. 80, no. 2, p. 021921, 2009.

[18] E. Lauga and T. Powers, "The hydrodynamics of swimming microorganisms," Reports on Progress in Physics, vol. 72, no. 9, p. 096601, 2009.

[19] R. Ananthakrishnan and A. Ehrlicher, "The forces behind cell movement," International Journal of Biological Sciences, vol. 3, no. 5, 2007.

[20] H. Lodish, A. Berk, C. A. Kaiser, M. Krieger, M. P. Scott, A. Bretscher, H. Ploegh, and P. Matsudaira, Molecular Cell Biology. W. H. Freeman, 6th ed., June 2007.

[21] T. Ishikawa, "Suspension biomechanics of swimming microbes," Journal of The Royal Society Interface, vol. 6, pp. 815 -834, Oct. 2009.

[22] U. B. Kaupp, N. D. Kashikar, and I. Weyand, "Mechanisms of sperm chemotaxis," Annual Review of Physiology, vol. 70, pp. 93-117, Mar. 2008.

[23] F. P. Bretherton and F. R. S. Rothschild, "Rheotaxis of spermatozoa," Proceedings of the Royal Society of London, Series B, vol. 153, Feb. 1961.

[24] H. C. Berg, "Motile behaviour of bacteria," Physics Today, vol. 53, no. 1, pp. 24-29, 2000.

[25] H. C. Berg, Random walks in biology. Princeton University Press, Sept. 1993.

[26] D. R. Mitchell, "The evolution of eukaryotic cilia and flagella as motile and sensory organelles," Advances in Experimental Medicine and Biology, vol. 607, 2007.

[27] K. S. Ralston, Z. Kabututu, J. H. Melehani, M. Oberholzer, and K. L. Hill, "The Trypanosoma brucei flagellum: Moving parasites in new directions," Annual Review of Microbiology, vol. 63, pp. 335-362, 2009.

[28] K. R. Matthews, "The developmental cell biology of Trypanosoma brucei," J Cell Sci, vol. 118, pp. 283-290, Jan. 2005.

[29] C. Branche, L. Kohl, G. Toutirais, J. Buisson, J. Cosson, and P. Bastin, "Conserved and specific functions of axoneme components in trypanosome motility," J Cell Sci, vol. 119, pp. 3443-3455, Aug. 2006.

[30] N. Heddergott, T. Krüger, A. Wei, E. Stellamans, S. Uppaluri, T. Pfohl, and M. Engstler, "Trypanosome motion represents an adaptation to the crowded environment of the vertebrate bloodstream.," In preparation.

[31] J. A. Rodríguez, M. A. Lopez, M. C. Thayer, Y. Zhao, M. Oberholzer, D. D. Chang, N. K. Kisalu, M. L. Penichet, G. Helguera, R. Bruinsma, K. L. Hill, and J. Miao, "Propulsion of African trypanosomes is driven by bihelical waves with alternating chirality separated by kinks," Proceedings of the National Academy of Sciences of the United States of America, Oct. 2009. 
[32] J. W. Shaevitz, J. Y. Lee, and D. A. Fletcher, "Spiroplasma swim by a processive change in body helicity," Cell, vol. 122, Sept. 2005.

[33] C. W. Wolgemuth, O. Igoshin, and G. Oster, "The motility of mollicutes," Biophysical Journal, vol. 85, pp. 828-842, Aug. 2003.

[34] C. Li, C. W. Wolgemuth, M. Marko, D. G. Morgan, and N. W. Charon, "Genetic analysis of spirochete flagellin proteins and their involvement in motility, filament assembly, and flagellar morphology," J. Bacteriol., vol. 190, pp. 5607-5615, Aug. 2008.

[35] A. Einstein, "Motion of suspended particles on the kinetic theory," Annalen der Physik, vol. 17, no. 3, pp. 549-560, 1905.

[36] D. Selmeczi, L. Li, L. I. Pedersen, S. F. Norrelykke, P. H. Hagedorn, S. Mosler, N. B. Larsen, E. C. Cox, and H. Flyvbjerg, "Cell motility as random motion: A review," The European Physical Journal Special Topics, vol. 157, no. 1, pp. 1-15, 2008.

[37] P. Kareiva and N. Shigesada, "Analyzing insect movement as a correlated random walk," Oecologia, vol. 56, no. 2/3, pp. 234-238, 1983.

[38] E. A. Codling, M. J. Plank, and S. Benhamou, "Random walk models in biology," Journal of The Royal Society Interface, vol. 5, no. 25, pp. 813-834, 2008.

[39] R. J. Nossal and G. H. Weiss, "A generalized Pearson random walk allowing for bias," Journal of Statistical Physics, vol. 10, pp. 245-253, Mar. 1974.

[40] D. S. Lemons, "Paul langevin's 1908 paper "On the theory of brownian motion [Sur la théorie du mouvement brownien,", C. R. Acad. Sci. (Paris) 146, 530-533 (1908)]," American Journal of Physics, vol. 65, no. 11, p. 1079, 1997.

[41] D. Brockmann, L. Hufnagel, and T. Geisel, "The scaling laws of human travel," Nature, vol. 439, pp. 462-465, Jan. 2006.

[42] M. C. Gonzalez, C. A. Hidalgo, and A. Barabasi, "Understanding individual human mobility patterns," Nature, vol. 453, pp. 779-782, June 2008.

[43] C. Sueur, "A Non-Lévy random walk in chacma baboons: What does it mean?," PLoS ONE, vol. 6, p. e16131, Jan. 2011.

[44] A. M. Edwards, R. A. Phillips, N. W. Watkins, M. P. Freeman, E. J. Murphy, V. Afanasyev, S. V. Buldyrev, M. G. E. da Luz, E. P. Raposo, H. E. Stanley, and G. M. Viswanathan, "Revisiting levy flight search patterns of wandering albatrosses, bumblebees and deer," Nature, vol. 449, pp. 1044-1048, Oct. 2007.

[45] A. M. Reynolds, J. L. Swain, A. D. Smith, A. P. Martin, and J. L. Osborne, "Honeybees use a lévy flight search strategy and odour-mediated anemotaxis to relocate food sources," Behavioral Ecology and Sociobiology, vol. 64, pp. 115-123, July 2009.

[46] H. Hirumi and K. Hirumi, "Continuous cultivation of Trypanosoma brucei blood stream forms in a medium containing a low concentration of serum protein without feeder cell layers," The Journal of Parasitology, vol. 75, Dec. 1989.

[47] H. Hirumi and K. Hirumi, "Axenic culture of African trypanosome bloodstream forms," Parasitology Today, vol. 10, no. 2, pp. 80-84, 1994. 
[48] K. L. Hill, "Biology and mechanism of trypanosome cell motility," Eukaryotic Cell, vol. 2, pp. 200-208, Apr. 2003.

[49] M. J. Miller, S. H. Wei, I. Parker, and M. D. Cahalan, "Two-photon imaging of lymphocyte motility and antigen response in intact lymph node," Science, vol. 296, June 2002.

[50] C. Gadelha, B. Wickstead, and K. Gull, "Flagellar and ciliary beating in trypanosome motility," Cell Motility and the Cytoskeleton, vol. 64, no. 8, pp. 629-643, 2007.

[51] S. Köster and T. Pfohl, "An in vitro model system for cytoskeletal confinement," Cell Motility and the Cytoskeleton, vol. 66, no. 10, pp. 771-776, 2009.

[52] S. Köster, D. Steinhauser, and T. Pfohl, "Brownian motion of actin filaments in confining microchannels," Journal of Physics: Condensed Matter, vol. 17, no. 49, pp. S4091-S4104, 2005.

[53] M. Rubinstein and R. H. Colby, Polymer Physics. Oxford University Press, USA, June 2003.

[54] J. Howard, Mechanics of Motor Proteins and the Cytoskeleton. Sunderland, MA 01375 USA.: Sinauer Associates, Inc, 2001.

[55] M. Ginger, N. Portman, and P. McKean, "Swimming with protists: Perception, motility and flagellum assembly," Nature Reviews Microbiology, vol. 6, no. 11, pp. 838-850, 2008.

[56] R. Gilad, A. Porat, and S. Trachtenberg, "Motility modes of spiroplasma melliferum BC3: a helical, wall-less bacterium driven by a linear motor," Molecular Microbiology, vol. 47, Feb. 2003.

[57] Y. Takano, K. Yoshida, S. Kudo, M. Nishitoba, and Y. Magariyama, "Analysis of small deformation of helical flagellum of swimming vibrio alginolyticus," JSME Int Journal. Ser C. Mech Systems, Mach Elem Manuf, vol. 46, no. 4, pp. 1241-1247, 2003.

[58] H. Takagi, M. J. Sato, T. Yanagida, and M. Ueda, "Functional analysis of spontaneous cell movement under different physiological conditions," PLoS ONE, vol. 3, p. e2648, July 2008.

[59] P. S. Lovely and F. W. Dahlquist, "Statistical measures of bacterial motility and chemotaxis," Journal of Theoretical Biology, vol. 50, no. 2, pp. 477 - 496, 1975.

[60] L. Li, S. F. Norrelykke, and E. C. Cox, "Persistent cell motion in the absence of external signals: A search strategy for eukaryotic cells," PLoS ONE, vol. 3, no. 5, 2008.

[61] F. Peruani and L. G. Morelli, "Self-propelled particles with fluctuating speed and direction of motion in two dimensions," Physical Review Letters, vol. 99, p. 010602, July 2007.

[62] A. Upadhyaya, J. Rieu, J. A. Glazier, and Y. Sawada, "Anomalous diffusion and non-Gaussian velocity distribution of hydra cells in cellular aggregates," Physica A: Statistical Mechanics and its Applications, vol. 293, pp. 549-558, Apr. 2001. 
[63] H. Risken, The Fokker-Planck equation: methods of solution and applications. Springer, Sept. 1996.

[64] V. Zaburdaev and H. Stark 2011. in preparation.

[65] P. E. Kloeden and E. Platen, Numerical Solution of Stochastic Differential Equations. Springer, Dec. 2010.

[66] D. M. Baron, K. S. Ralston, Z. P. Kabututu, and K. L. Hill, "Functional genomics in trypanosoma brucei identifies evolutionarily conserved components of motile flagella," J Cell Sci, vol. 120, pp. 478-491, Feb. 2007.

[67] W. Masocha, M. E. Rottenberg, and K. Kristensson, "Migration of African trypanosomes across the blood-brain barrier," Physiology \& Behavior, vol. 92, pp. 110114, Sept. 2007.

[68] P. Dash, "Cell migration lab." http:/ / www.reading.ac.uk/cellmigration/matrix.htm, 2010.

[69] R. M. Kuntz and W. M. Saltzman, "Neutrophil motility in extracellular matrix gels: mesh size and adhesion affect speed of migration.," Biophysical Journal, vol. 72, Mar. 1997.

[70] B. A. Roeder, K. Kokini, J. E. Sturgis, J. P. Robinson, and S. L. Voytik-Harbin, “Tensile mechanical properties of three-dimensional type i collagen extracellular matrices with varied microstructure," Journal of Biomechanical Engineering, vol. 124, Apr. 2002.

[71] "Physical properties of dextran." http://www.dextran.net/dextran-physicalproperties.html, 2010.

[72] V. Tirtaatmadja, D. E. Dunstan, and D. V. Boger, "Rheology of dextran solutions," Journal of Non-Newtonian Fluid Mechanics, vol. 97, pp. 295-301, Feb. 2001.

[73] I. Y. Wong, M. L. Gardel, D. R. Reichman, E. R. Weeks, M. T. Valentine, A. R. Bausch, and D. A. Weitz, "Anomalous diffusion probes microstructure dynamics of entangled f-actin networks," Physical Review Letters, vol. 92, Apr. 2004.

[74] M. L. Gardel, M. T. Valentine, J. C. Crocker, A. R. Bausch, and D. A. Weitz, "Microrheology of entangled F-Actin solutions," Physical Review Letters, vol. 91, p. 158302 , Oct. 2003.

[75] L. A. Fanti and E. D. Glandt, "Partitioning of spherical particles into fibrous matrices : 2. Monte Carlo simulation," Journal of Colloid and Interface Science, vol. 135, pp. 396-404, Mar. 1990.

[76] W. M. Saltzman, M. L. Radomsky, K. J. Whaley, and R. A. Cone, "Antibody diffusion in human cervical mucus.," Biophysical Journal, vol. 66, Feb. 1994.

[77] W. R. Schneider and R. N. Doetsch, "Effect of viscosity on bacterial motility," Journal of Bacteriology, vol. 117, Feb. 1974.

[78] H. C. Berg and L. Turner, "Movement of microorganisms in viscous environments," Nature, vol. 278, Mar. 1979. 
[79] Y. Magariyama and S. Kudo, "A mathematical explanation of an increase in bacterial swimming speed with viscosity in linear-polymer solutions.," Biophysical Journal, vol. 83, Aug. 2002.

[80] T. Atsumi, Y. Maekawa, T. Yamada, I. Kawagishi, Y. Imae, and M. Homma, "Effect of viscosity on swimming by the lateral and polar flagella of Vibrio alginolyticus," Journal of Bacteriology, vol. 178, Aug. 1996.

[81] E. Stellamanns, Cell Motility in Microfluidic Environments. PhD thesis, Mathematics and Natural Sciences of the Georg-August-Universität Göttingen, Max Planck Institute for Dynamics and Self Organization, Goettingen, 2011.

[82] N. Heddergott, Zellbiologische Aspekte der Motilität von Trypanosoma brucei unter Berücksichtigung der Interaktion mit der Mikroumwelt. PhD thesis, University of Würzburg, Würzburg, 2011.

[83] S. Köster, Y. Lin, H. Herrmann, and D. A. Weitz, "Nanomechanics of vimentin intermediate filament networks," Soft Matter, vol. 6, no. 9, p. 1910, 2010.

[84] P. Cicuta and A. M. Donald, "Microrheology: a review of the method and applications," Soft Matter, vol. 3, no. 12, p. 1449, 2007.

[85] T. A. Waigh, "Microrheology of complex fluids," Reports on Progress in Physics, vol. 68, pp. 685-742, Mar. 2005.

[86] D. Velegol and F. Lanni, "Cell traction forces on soft biomaterials. I. Microrheology of type i collagen gels.," Biophysical Journal, vol. 81, Sept. 2001.

[87] A. P. Berke, L. Turner, H. C. Berg, and E. Lauga, "Hydrodynamic attraction of swimming microorganisms by surfaces," Physical Review Letters, vol. 101, pp. 038102-4, July 2008.

[88] R. Zhang, L. Turner, and H. C. Berg, "The upper surface of an Escherichia coli swarm is stationary," Proceedings of the National Academy of Sciences, Dec. 2009.

[89] E. Lauga, W. R. DiLuzio, G. M. Whitesides, and H. A. Stone, "Swimming in circles: Motion of bacteria near solid boundaries," Biophysical Journal, vol. 90, pp. 400-412, Jan. 2006.

[90] J. Hill, O. Kalkanci, J. L. McMurry, and H. Koser, "Hydrodynamic surface interactions enable Escherichia coli to seek efficient routes to swim upstream," Physical Review Letters, vol. 98, Feb. 2007.

[91] D. Steinhauser, Actin Filaments and Bundles in Flow. PhD Thesis, Mathematics and Natural Sciences of the Georg-August-Universität Göttingen, Göttingen, 2008.

[92] M. Abkarian and A. Viallat, "Dynamics of vesicles in a Wall-Bounded shear flow," Biophysical Journal, vol. 89, pp. 1055-1066, Aug. 2005.

[93] M. Abkarian and A. Viallat, "Vesicles and red blood cells in shear flow," Soft Matter, vol. 4, no. 4, p. 653, 2008.

[94] J. Elgeti, U. B. Kaupp, and G. Gompper, "Hydrodynamics of sperm cells near surfaces," Biophysical Journal, vol. 99, no. 4, pp. 1018-1026, 2010. 
[95] J. Elgeti and G. Gompper, "Self-propelled rods near surfaces," EPL (Europhysics Letters), vol. 85, no. 3, p. 38002, 2009.

[96] D. Daintillan, E. S. Shaqfeh, and E. Darve, "Effect of flexibility on the Shear-Induced migration of Short-Chain polymers in parabolic channel flow," Journal of Fluid Mechanics, vol. 557, pp. 297-306, 2006.

[97] D. Ausserré, J. Edwards, J. Lecourtier, H. Hervet, and F. Rondelez, "Hydrodynamic thickening of depletion layers in colloidal solutions," Europhysics Letters (EPL), vol. 14, no. 1, pp. 33-38, 1991.

[98] J. Depablo, H. Ottinger, and Y. Rabin, "Hydrodynamic changes of the depletion layer of dilute polymer solutions near a wall," AIChE journal, vol. 38, no. 2, pp. 273283, 1992.

[99] O. B. Usta, J. E. Butler, and A. J. C. Ladd, "Flow-induced migration of polymers in dilute solution," Physics of Fluids, vol. 18, no. 3, p. 031703, 2006.

[100] J. J. D. Pablo, H. C. Öttinger, and Y. Rabin, "Hydrodynamic changes of the depletion layer of dilute polymer solutions near a wall," AIChE Journal, vol. 38, pp. 273-283, Feb. 1992.

[101] M. Parsons and B. Nielsen, "Active transport of 2-deoxy-d-glucose in Trypanosoma brucei procyclic forms," Molecular and Biochemical Parasitology, vol. 42, pp. 197-203, Sept. 1990.

[102] M. Ralser, M. M. Wamelink, E. A. Struys, C. Joppich, S. Krobitsch, C. Jakobs, and H. Lehrach, "A catabolic block does not sufficiently explain how 2-deoxy-dglucose inhibits cell growth," Proceedings of the National Academy of Sciences, vol. 105, pp. $17807-17811$, Nov. 2008.

[103] M. Nagai, H. Asai, and H. Fujita, "Contraction and extension of vorticella and its mechanical characterization under flow loading," Biomicrofluidics, vol. 4, no. 3, p. 034109, 2010.

[104] Y. Gebremichael, G. S. Ayton, and G. A. Voth, "Mesoscopic modeling of bacterial flagellar microhydrodynamics," Biophysical Journal, vol. 91, Nov. 2006.

[105] I. H. Riedel-Kruse, A. Hilfinger, J. Howard, and F. Jülicher, "How molecular motors shape the flagellar beat," HFSP Journal, vol. 1, Sept. 2007.

[106] N. Nève, S. S. Kohles, S. R. Winn, and D. C. Tretheway, "Manipulation of suspended single cells by microfluidics and optical tweezers," Cellular and Molecular Bioengineering, vol. 3, Sept. 2010.

[107] S. Yamada, "Mechanics of living cells measured by laser tracking microrheology," Biophysical Journal, vol. 78, pp. 1736-1747, Apr. 2000.

[108] M. Dao, J. Li, and S. Suresh, "Molecularly based analysis of deformation of spectrin network and human erythrocyte," Materials Science and Engineering: C, vol. 26, pp. 1232-1244, Sept. 2006. 
[109] D. A. Fedosov, B. Caswell, S. Suresh, and G. E. Karniadakis, "Quantifying the biophysical characteristics of plasmodium-falciparum-parasitized red blood cells in microcirculation," Proceedings of the National Academy of Sciences, vol. 108, pp. 35 -39, Jan. 2011.

[110] "Blood function and composition - summary." http://www.virtualmedicalcentre.com/anatomy.asp?sid=30.

[111] R. E. Wells and E. W. Merrill, "Shear rate dependence of the viscosity of whole blood and plasma," Science, vol. 133, pp. 763 -764, Mar. 1961.

[112] J. L. McWhirter, H. Noguchi, and G. Gompper, "Flow-induced clustering and alignment of vesicles and red blood cells in microcapillaries," Proceedings of the $\mathrm{Na}$ tional Academy of Sciences, vol. 106, pp. 6039 -6043, Apr. 2009.

[113] P. Cherukat, J. McLaughlin, and A. Graham, "The inertial lift on a rigid sphere translating in a linear shear flow field," International Journal of Multiphase Flow, vol. 20, pp. 339-353, Apr. 1994.

[114] J. Park, S. Song, and H. Jung, "Continuous focusing of microparticles using inertial lift force and vorticity via multi-orifice microfluidic channels," Lab on a Chip, vol. 9, no. 7, p. 939, 2009.

[115] I. Cantat and C. Misbah, "Lift force and dynamical unbinding of adhering vesicles under shear flow," Physical Review Letters, vol. 83, p. 880, July 1999.

[116] G. Coupier, B. Kaoui, T. Podgorski, and C. Misbah, “Noninertial lateral migration of vesicles in bounded poiseuille flow," Physics of Fluids, vol. 20, pp. 111702-4, Nov. 2008.

[117] S. Messlinger, B. Schmidt, H. Noguchi, and G. Gompper, "Dynamical regimes and hydrodynamic lift of viscous vesicles under shear," Physical Review E, vol. 80, p. 011901, July 2009.

[118] M. Faivre, M. Abkarian, K. Bickraj, and H. A. Stone, "Geometrical focusing of cells in a microfluidic device: an approach to separate blood plasma," Biorheology, vol. 43, no. 2, 2006.

[119] M. Faivre, Gouttes, vésicules et globules rouges: Deformabilité et comportement sous écoulement. PhD thesis, Université Joseph Fourier, Grenoble.

[120] P. Olla, "The role of tank-treading motions in the transverse migration of a spheroidal vesicle in a shear flow," Journal of Physics A: Mathematical and General, vol. 30, pp. 317-329, Jan. 1997.

[121] A. A. S. Bhagat, H. Bow, H. W. Hou, S. J. Tan, J. Han, and C. T. Lim, "Microfluidics for cell separation," Medical \& Biological Engineering E Computing, vol. 48, Oct. 2010.

[122] A. Jain and L. L. Munn, "Determinants of leukocyte margination in rectangular microchannels," PLoS ONE, vol. 4, no. 9, p. e7104, 2009.

[123] L. L. Munn and M. M. Dupin, "Blood cell interactions and segregation in flow," Annals of biomedical engineering, vol. 36, Apr. 2008. 
[124] S. H. Holm, J. P. Beech, M. P. Barrett, and J. O. Tegenfeldt, "Separation of parasites from human blood using deterministic lateral displacement," Lab on a Chip, vol. 11, no. 7, p. 1326, 2011.

[125] H. W. Hou, A. A. S. Bhagat, A. G. L. Chong, P. Mao, K. S. W. Tan, J. Han, and C. T. Lim, "Deformability based cell margination - simple microfluidic design for malaria-infected erythrocyte separation," Lab on a Chip, vol. 10, no. 19, p. 2605, 2010.

[126] K. C. Chaw, M. Manimaran, F. E. H. Tay, and S. Swaminathan, "Matrigel coated polydimethylsiloxane based microfluidic devices for studying metastatic and nonmetastatic cancer cell invasion and migration," Biomedical Microdevices, vol. 9, Aug. 2007. 



\section{Publications}

Sravanti Uppaluri, Jan Nagler, Eric Stellamanns, Niko Heddergott, Stephan Herminghaus, Markus Engstler, and Thomas Pfohl. "Impact of microscopic motility on the swimming behavior of parasites: stiffer trypanosomes are more directional" Accepted to PLoS Computational Biology, 2011.

Vasily Zaburdaev, Sravanti Uppaluri, Rudolf Friedrich, Markus Engstler, Thomas Pfohl, and Holger Stark, "Langevin dynamics deciphers the motility pattern of swimming parasites," Physical Review Letters, vol. 106, no. 20, p. 208103, May. 2011.

Sravanti Uppaluri, Eric Stellamanns, Niko Heddergott, Stephan Herminghaus, Markus Engstler, and Thomas Pfohl. "Physics of parasite motility in flow" In preparation.

Niko Heddergott, Timothy Krüger, Ai Wei, Eric Stellamanns, Sravanti Uppaluri, Thomas Pfohl, and Markus Engstler. "Trypanosome motion represents an adaptation to the crowded environment of the vertebrate bloodstream" In preparation.

\section{Conferences}

2009

DPG Frühjahrstagung - Dresden, Germany (talk, poster)

2009

Dynamics Days Europe - Göttingen, Germany (poster)

2010

Biophysical Society Meeting - San Francisco, USA (talk)

2010

2011

DPG Frühjahrstagung - Regensburg, Germany (talk, poster)

DPG Frühjahrstagung - Dresden, Germany (talk) 



\section{Acknowledgements}

I am grateful to Prof. Dr. Thomas Pfohl for his unfailing guidance throughout this journey. His scientific expertise and patience during our long discussions have been instrumental in helping complete this work. I am very lucky indeed to have found a mentor from whom I have learned many lessons in science and otherwise that I will carry with me for many days to come.

I would like to thank Prof. Dr. Stephan Herminghaus, for providing me the opportunity to work at the Max Planck Institute for Dynamics and Self-Organization. His enthusiasm for discovery has provided an ideal environment for scientific pursuit.

I thank my thesis committee members, Prof. Dr. Marc Timme and Prof. Dr. Detlev Schild for their guidance and encouragement during committee meetings.

This work would not have been possible were it not for fruitful collaborations with Dr. Jan Nagler. Jan, I will always remember our combined struggles to understand what was going on! My work with Dr. Vasily Zaburdaev and Prof. Dr. Holger Stark has also been very productive. Late night emails and chats over different time zones with Vasily were well worth the result! Both Prof. Dr. Markus Engstler and Niko Heddergott have been a great support and helped with many of our trypanosome problems! Our collaboration has been extremely valuable. My thanks also go to Andreas Zöttl for discussions on my results of trypanosomes in flow.

I am grateful to Melanie Scharfenstein for always being willing to provide blood samples on short notice. Monica Teuteberg has really been a remarkable support throughout our stay in Germany. I will never forget her kindness whenever I felt lost. The technical support from Thomas Eggers, Markus Benderoth, Udo Krafft, Wolf Keiderling, Dr. Kris Hantke, and Sybille Naegle has been invaluable to my work here. Antje Erdmann and Michaela Boettcher have been incredibly efficient, and I am obliged to them for facilitating all things GGNB.

I would like to thank my partner in 'trypanosome-crime', Eric Stellamanns for many discussions, collaborative efforts, and a wonderful friendship. I will forever remember Semra Oeztuerk for being a great technician, but more importantly for being an amazing friend. I wish you and Ismail the best in life. I will never forget my extremely talented friend and office-mate Sonia Utermann. Sonia, thank you for your company - you are a cici-kiz, ! Konstantina, Daniel and Leonardo - I wish there was more time and more tiramisu to eat!

Many shared discussions, frustrations and laughs with Dagmar, Heather, Siddharth, Adriana, Rolf, Axel, and Raphael have been a genuine support throughout. I would like to thank all members of our department for shared lunches, coffee, and hallway discussions including Chih-Wei, Karthik, Klaus, Daniel, Johannes, Martin, Pramod, Tuul, Venkat, Krish, Martha, Christopher and Jürgen. A special mention is required for my 
doctor-family: Ulrike for being there for me and Max for being a wonderful play-mate.

Thanks to Rajiv, Deepa and Devan for coming to visit us all the way from Ottawa, the short time we spent together (30 seconds!) in Göttingen was precious and memorable. Our stay in Goettingen has been a memorable one with many many good times thanks to unforgettable bonds formed with our friends: Harish, Shweta, Vimal, Atul, Srinivas, Soujanya, Gopal \& co., Sailaja, Mayur, Eashwar, Aniket, Swati, Sarath, Anjali, Sonia, Somdatta, and many more.

I am grateful to my parents for giving me a life of discovery, and abundant love. I am thankful to my mother for showing me the importance of the small things in life that give great joy, and to my father for showing me by example the impact of hard work and integrity. My akka and bava have been second parents to me at times, and the best of friends at other times whenever I needed them without exception. Thanks to anna for showing me what uninhibited generosity is, and to Lien for always making me smile with her warm heartedness. I have been blessed with three bright rays of sun shine my nieces: Meena, Medha and Manu to whom I owe many smiles and moments of pure happiness. I am thankful to my in-laws, maa and mamayya for their love, support and especially for making me their own. I am indebted to my Ram-peddananagaru, for all the Chinese checkers games and help with undergrad set theory problems - which I am sure in some way set me on my path. And to my husband, Shashi - his iridescent spirit, quiet resolve, and sincerity have been and always will be a source of strength to me in our journey together. I am grateful to him for giving me all there is to give. 


\section{Curriculum Vitae}

\section{Personal Information}

$\begin{array}{ll}\text { Name: } & \text { Sravanti Uppaluri } \\ \text { Date of Birth: } & \text { 3 May 1980 } \\ \text { Place of Birth: } & \text { Hyderabad, India } \\ \text { Nationality: } & \text { Canadian }\end{array}$

\section{Education and Employment History}

2008-Present Doctoral candidate, Max Planck Institute for Dynamics and Self-Organization - Göttingen, Germany

2007-2008 Research assistant, Max Planck Institute for Dynamics and Self-Organization - Göttingen, Germany

2005-2007 Project manager, Aurigene (Biopharmaceutical Company) Bangalore, India

2002-2004 Master of Applied Science, University of Toronto - Canada

1998-2002 Bachelor of Life Science, with honors, McMaster University - Hamilton, Canada

1998

Secondary School Certificate, with honors, Bluevale Collegiate Institute - Waterloo, Canada 